Approved and certified by L. Wallace, University of Dayton, Graduate Academic Affairs

\title{
LOW REYNOLDS NUMBER EXPERIMENTAL AERODYNAMIC VERIFICATION OF SCALED AND LEWICE SIMULATED ICE ACCRETIONS IN SLD CONDITIONS
}

\author{
Thesis \\ Submitted to \\ The School of Engineering of the \\ UNIVERSITY OF DAYTON
}

\author{
In Partial Fulfillment of the Requirements for \\ The Degree of \\ Master of Science in Aerospace Engineering
}

By

Eric Joseph Insana

University of Dayton

Dayton, Ohio

August 2020

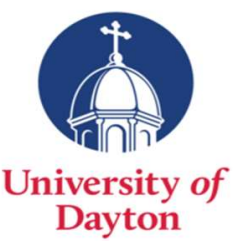




\section{LOW REYNOLDS NUMBER EXPERIMENTAL AERODYNAMIC VERIFICATION OF}

\section{SCALED AND LEWICE SIMULATED ICE ACCRETIONS IN SLD CONDITIONS}

Name: Insana, Eric

APPROVED BY:

Dr. Sidaard Gunasekaran

Advisory Committee Chairman

Assistant Professor

Mechanical and Aerospace Engineering
Dr. Joshua Heyne

Committee Member

Assistant Professor

Mechanical and Aerospace Engineering
Dr. Markus Rumpfkeil

Committee Member

Associate Professor

Mechanical and Aerospace Engineering

Robert J. Wilkens, Ph.D., P.E.

Associate Dean for Research and Innovation

Professor

School of Engineering
Dr. Jenching Tsao

Committee Member

Principal Research Scientist

Ohio Aerospace Institute
Eddy M. Rojas, Ph.D., M.A., P.E.

Dean, School of Engineering 


\begin{abstract}
LOW REYNOLDS NUMBER EXPERIMENTAL AERODYNAMIC VERIFICATION OF SCALED AND LEWICE SIMULATED ICE ACCRETIONS IN SLD CONDITIONS
\end{abstract}

Name: Insana, Eric Joseph

University of Dayton

Advisor: Dr. Sidaard Gunasekaran

Ice accretion is a primary operational flight hazard for which all FAA certified aircraft are evaluated. Due to the geometric limitation of icing research tunnels and the required icing studies performed prior to flight tests, a recommended scaling method by Anderson and Tsao is utilized to adapt icing tunnel parameters (such as the flow temperature, the test section flow velocity, the water droplet median volume diameter (MVD), the liquid water content (LWC), and the ice accretion time, $\tau$, etc.), along with model scaling to ensure the ice accretion collected is representative of the full-scale ice formation. Previous results have indicated good geometric agreement between the ice accretions formed on a full-scale model and the ice accretions on a scaled model, using the recommended scaling method under Title 14 of the Code of Federal Regulations (CFR), Part 25, Appendix $\mathrm{C}$ envelope. However, with the addition of the Appendix $\mathrm{O}$ envelope, which includes a large range of Supercooled Large Drop (SLD) conditions, the scaling method needed to be revised. SLD conditions refer to large median volume diameter (MVD) droplet conditions ranging from $50 \mu \mathrm{m}$ to greater than $500 \mu \mathrm{m}$ in the Appendix $\mathrm{O}$ envelope and generate larger frontal ice shape variation along with larger ice accretion further aft on a model.

Prior work by Anderson and Tsao evaluating the use of the ice shape scaling method for a limited range of SLD conditions, up to $190 \mu \mathrm{m}$, was based on geometric similitude of the frontal ice shape between the full-scale and scale collected ice accretions within allowable tolerances. The geometries of the full-scale and scale model collected ice accretions show significantly greater geometric variation under SLD conditions than Appendix C conditions, especially in the feather 
region, the ice accretions aft of the main formation on the leading edge. This brings into question the scaling methods ability to preserve the aerodynamics associated with the full-scale ice shape including changes in aerodynamic coefficients and its associated trends, and the mean and fluctuating velocity components in the flow field. The presented investigation provides insight into the current scaling method's effective use for SLD conditions based on aerodynamics through experimental evaluation of the lift and drag coefficients, the mean and fluctuating components of the leading edge and near wake flow fields, and the coherent structures present in these same flow field regions.

The computation software, LEWICE, predicts the ice formation on aircraft surfaces under a wide range of icing conditions. Literature exists on evaluation of LEWICE simulated ice accretions based on geometric similitude. However, minimal research has been performed to evaluate and verify the preservation of aerodynamics by the LEWICE predicted ice accretions, especially under SLD conditions. The LEWICE software does not predict any feather region ice accretions. As such, significant differences are seen between the ice accretions from experimental investigations and the ones predicted by LEWICE under specified SLD conditions. The research presented in this thesis provides insight into the LEWICE software's ability to predict an ice shape which is capable of recreating the aerodynamics and aerodynamic penalties associated with icing under SLD conditions. This includes experimental evaluation of the lift and drag coefficients, the mean and fluctuating components of the leading edge and near wake flow fields, and the coherent structures present in these same flow field regions.

The experimentation performed for aerodynamic evaluation was conducted at the University of Dayton Low Speed Wind Tunnel (UD-LSWT). Force-based experimentation was performed to study the lift and drag coefficients of each ice shape across a range of angles of attack. This allowed for deviations in stall angle, zero-lift angle of attack, and drag increase from a noniced baseline NACA0012 to be compared across the three different ice accretions: the LEWICE ice shape, the full-scale ice shape, and the scaled ice shape. Ice shapes collected with two different 
stagnation freezing fractions $\left(n_{0}=0.3\right.$ and $\left.n_{0}=0.5\right)$ were compared through the experimentation in the UD-LSWT. The stagnation freezing factor has a large effect on the geometry of the ice shape. Therefore, evaluating ice shapes collected at different stagnation freezing fractions allows for significantly different horn shapes to be studied for verification.

Particle Image Velocimetry (PIV) was performed on the leading edge and near wake regions of the iced airfoils to evaluate the flow dynamics of the ice accretions. The flow field dynamics were compared based on evaluation of the mean and fluctuating components of velocity. The mean component was studied through evaluating the momentum deficit in the wake, the flow separation on the upper and lower surfaces of the airfoil, the mean streamwise velocity, and the vorticity. The fluctuating component was studied through Reynolds stress. Along with these flow field parameters, a modal analysis of the flow fields was performed using Proper Orthogonal Decomposition (POD) to evaluate the presence of coherent structures in the flow and their corresponding length scales. This allowed for the LEWICE software and ice shape scaling method to be evaluated for the preservation of the aerodynamics associated with the full-scale ice shape to be evaluated.

The results of the low Reynolds number aerodynamic evaluation showed that ice accretions gathered on the scaled model inconsistently replicates the aerodynamics created by the ice accretions in the full-scale model for the given conditions. The stagnation freezing fraction was the primary difference between the scaled ice accretion that successfully replicated the full-scale aerodynamics, as in the case of $n_{0}=0.3$, and the scaled ice accretion that did not, as in the case of $n_{0}=0.5$. This suggests a possible stagnation freezing fraction constraint of the scaling method's use for SLD conditions. The LEWICE software consistently predicted an ice shape that was aerodynamically uncharacteristic of the full-scale ice accretion, therefore failing aerodynamic verification for the SLD conditions studied. 


\section{ACKNOWLEDGEMENTS}

I would like to start by thanking Dr. Sidaard Gunasekaran, my advisor, for continued support and guidance throughout my education at the University of Dayton and this thesis. Dr. Gunasekaran has been a great advisor, professor, and a friend. I will never take for granted how dedicated he is to his students.

I would also like to express my appreciation to all the personnel at the NASA Glenn Research Center who have helped throughout the completion of this thesis. I would like to specifically thank Luis Beltran, my mentor through the NASA Glenn PATHWAYS program, for

the opportunity to continue my education and the chance to continue my dreams through working at NASA.

Special thanks go to Jielong "Jacky" Cai and Michael Mongin for their support in the UDLSWT and for assistance with processing the large amount of PIV images collected in this thesis. Along with Jacky and Mike, I would like to thank Jen Colborn for her continued help and support throughout the thesis process.

Finally, I would like to thank my family for their continued support. I would not have been able to be where I am today without their help and guidance. 


\section{TABLE OF CONTENTS}

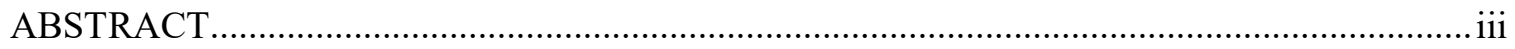

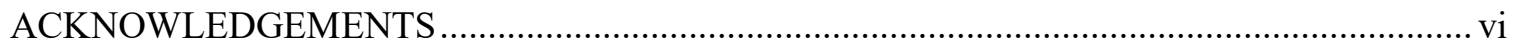

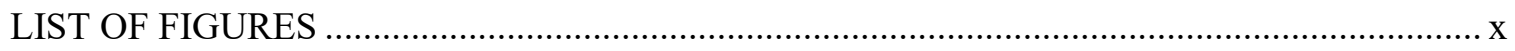

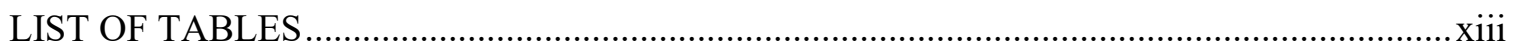

LIST OF ABBREVIATIONS AND NOMENCLATURE ..................................................... xiv

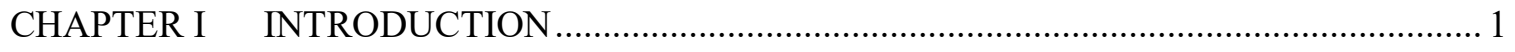

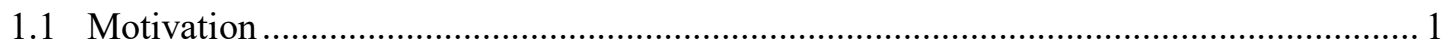

1.1.1 SLD Aerodynamic Verification of the Recommended Ice shape Scaling Method. 1

1.1.2 SLD Aerodynamics Verification of LEWICE ...................................................... 3

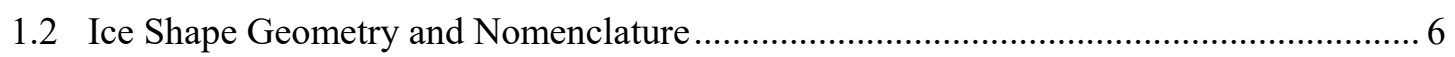

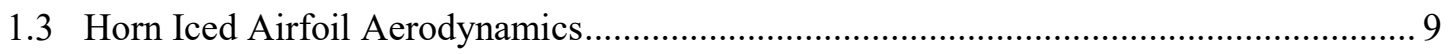

1.4 Ice Shape Scaling Method and Previous SLD Verification ........................................... 12

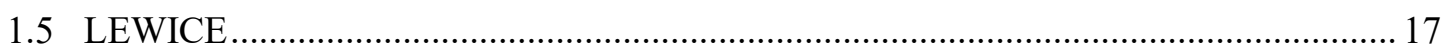

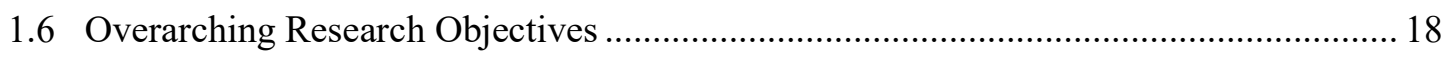

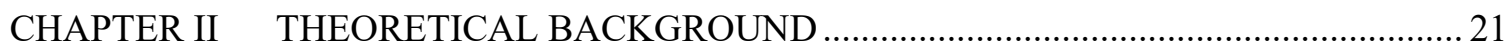

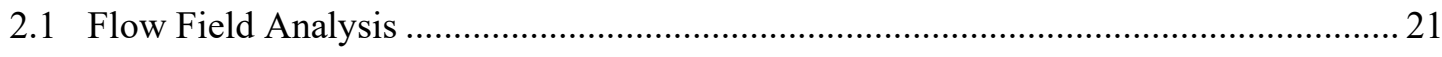

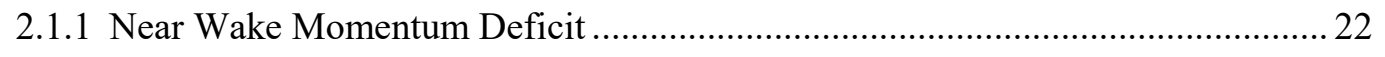

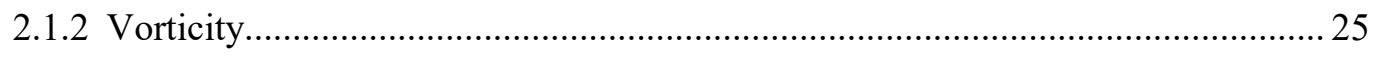

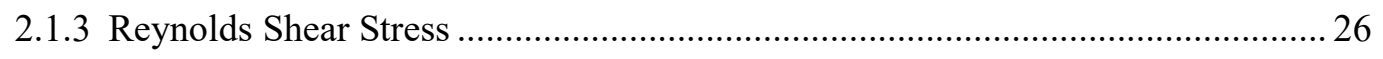

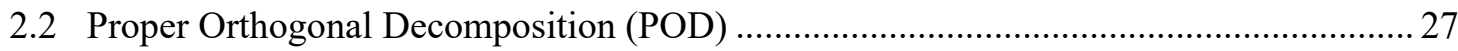

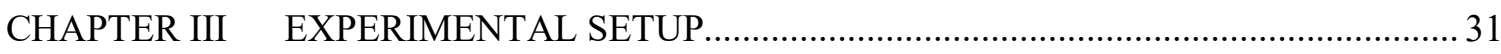

3.1 University of Dayton Low Speed Wind Tunnel (UD-LSWT ….................................... 31

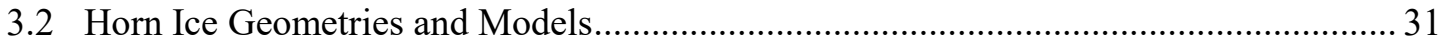

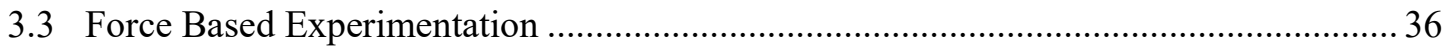

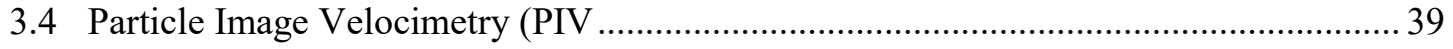

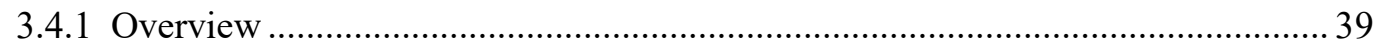

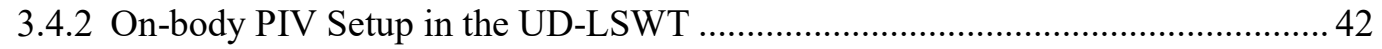

3.4.3 Free Sheer Layer PIV Setup in UD-LSWT ........................................................ 45 


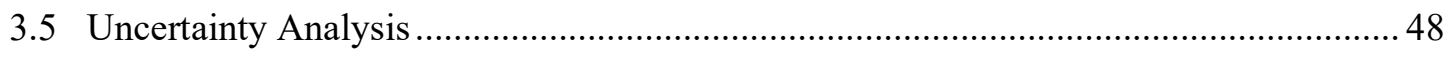

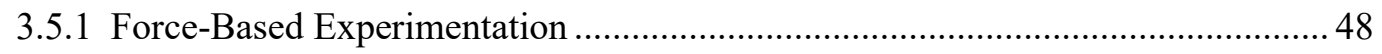

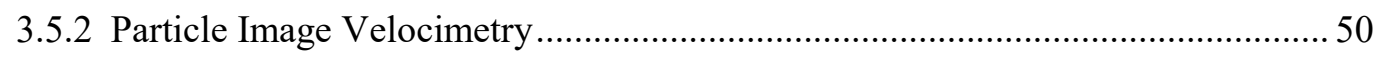

CHAPTER IV FORCE BASED EXPERIMENTATION AND MOMENTUM DEFICIT

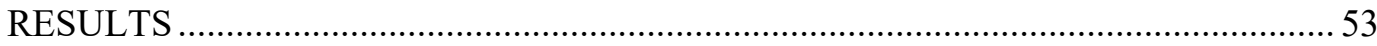

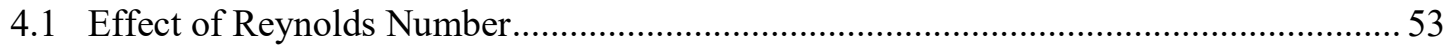

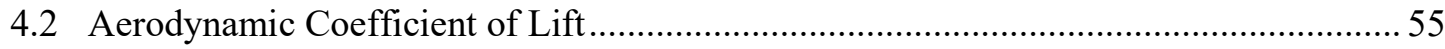

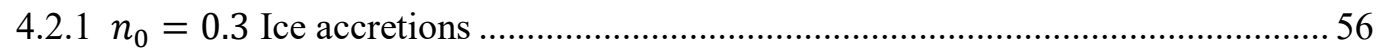

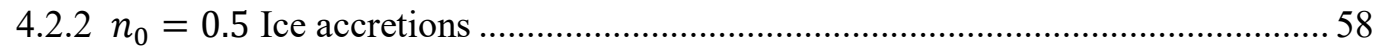

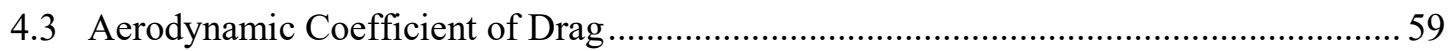

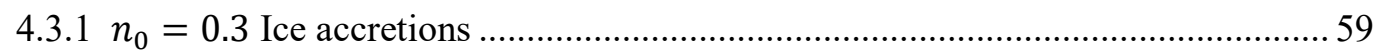

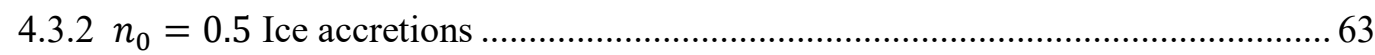

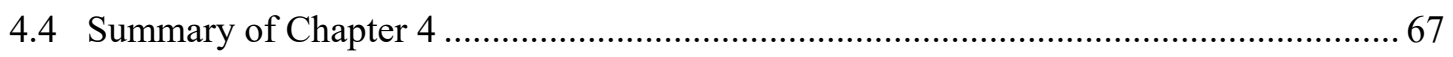

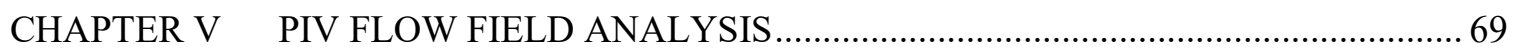

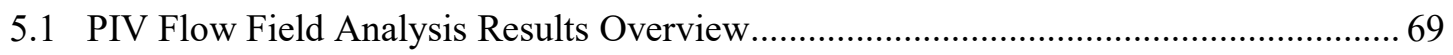

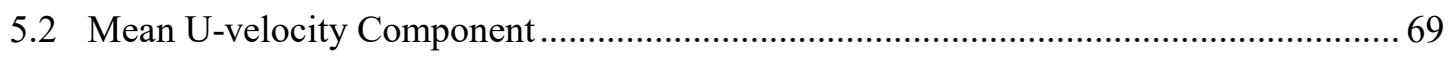

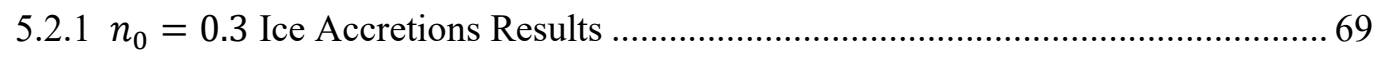

$5.2 .2 n_{0}=0.3$ Ice Accretions Summary ……....................................................... 73

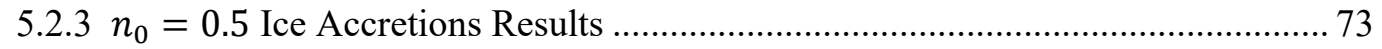

$5.2 .4 n_{0}=0.5$ Ice Accretions Summary …….......................................................... 76

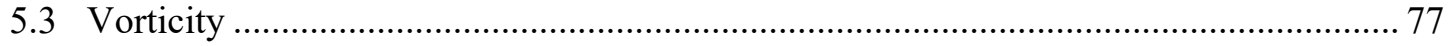

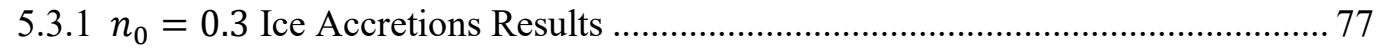

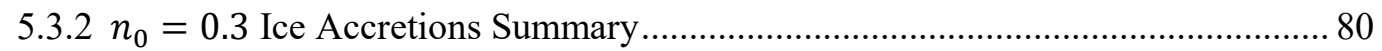

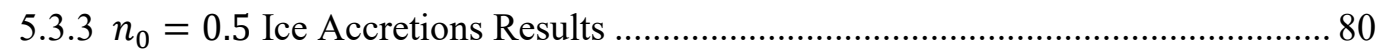

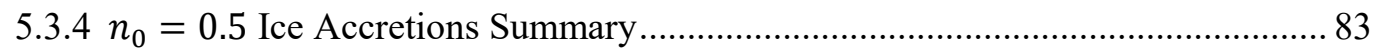

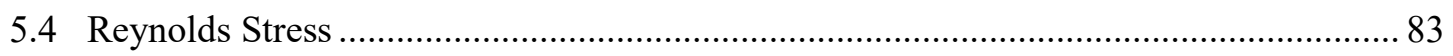

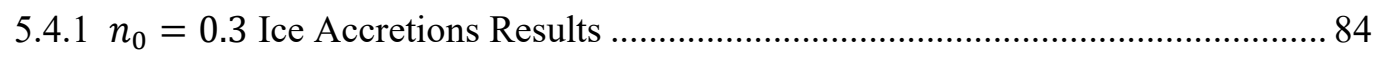

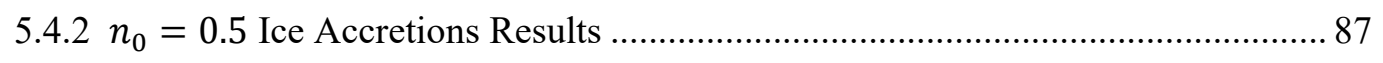

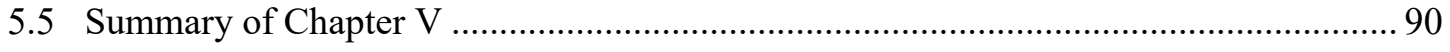

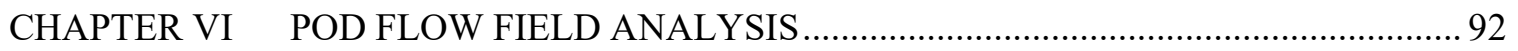

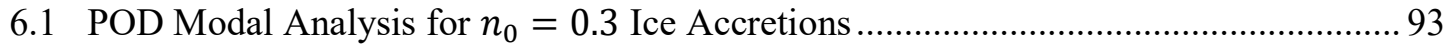

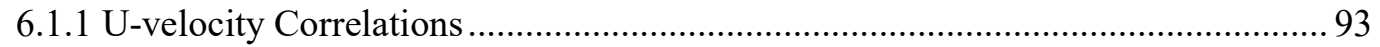




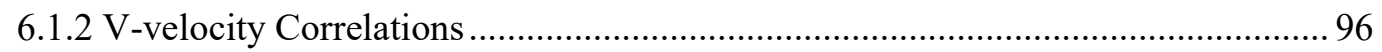

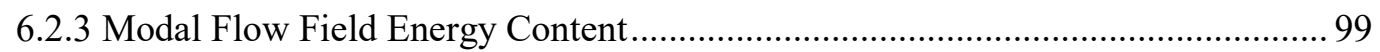

6.2 POD Modal Analysis for $n_{0}=0.5$ Ice Accretions ….................................................. 102

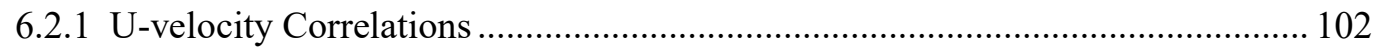

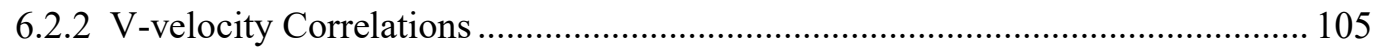

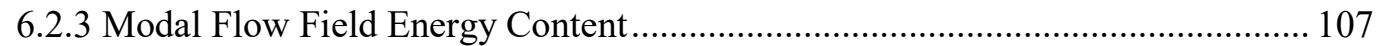

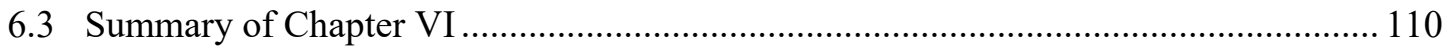

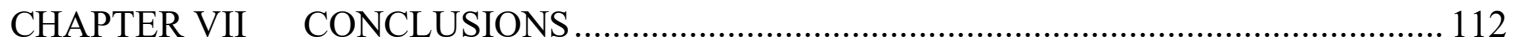

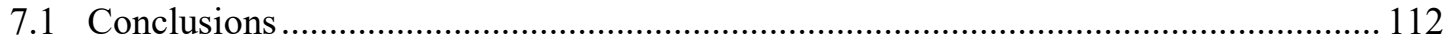

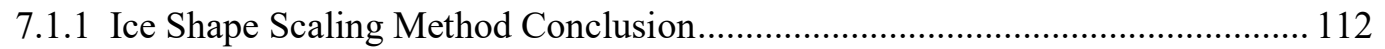

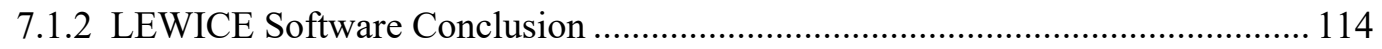

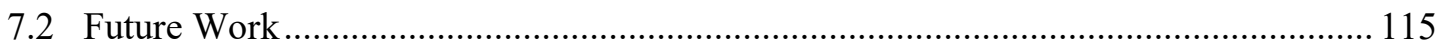

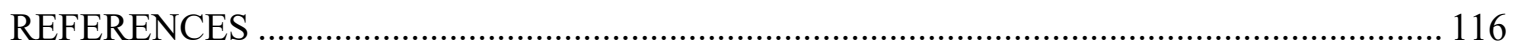

APPENDICES

A. LEWICE Ice Shape Input Files Based on Full-Scale Icing Parameters.......................... 120

B. MVD 170 $\mu$ m NASA Glenn Icing Research Tunnel Droplet Distribution Data .............. 123 


\section{LIST OF FIGURES}

Figure 1: Swept wing leading edge section in NASA Glenn IRT ........................................... 4

Figure 2: Primary ice shape regions of a given ice accretion in Appendix $\mathrm{C}$ conditions ............... 6

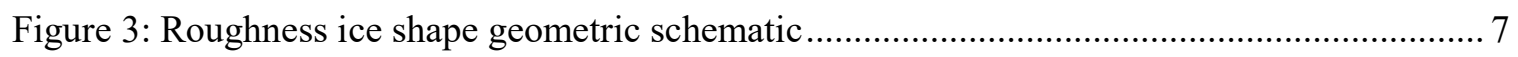

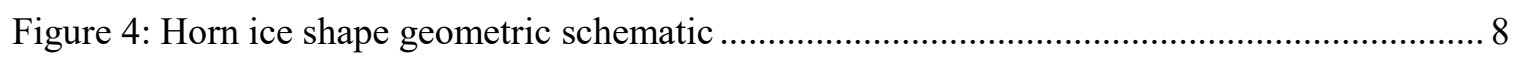

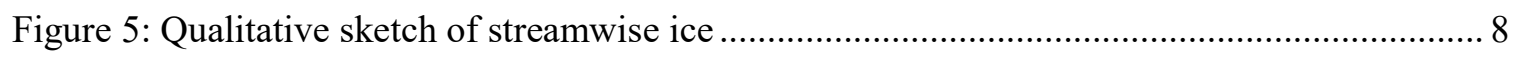

Figure 6: Simulated quarter-round spanwise-ridge ice …..................................................... 9

Figure 7: Instantaneous laminar separation bubble flow field schematic of a flat plate with an induced adverse pressure gradient at point $\mathrm{S}$, with corresponding pressure

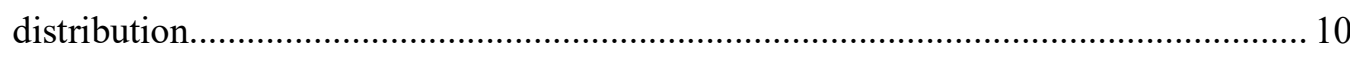

Figure 8: Upper surface separation bubble on a NACA 0012 schematic ................................... 11

Figure 9: Separation streamlines on a NACA 0012 airfoil with a simulated horn accretion......... 11

Figure 10: Scaled and full-scale ice accretion used in the verification for Appendix C

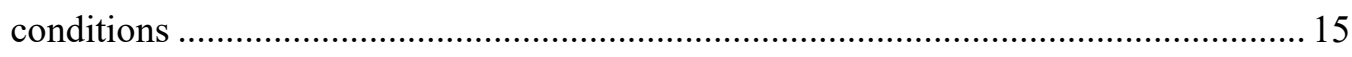

Figure 11: Scaled and full-scale ice accretion used in the verification for SLD conditions .......... 16

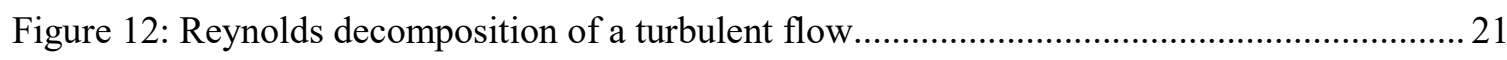

Figure 13: Flow around a body in a two-dimensional plane, control volume for obtaining

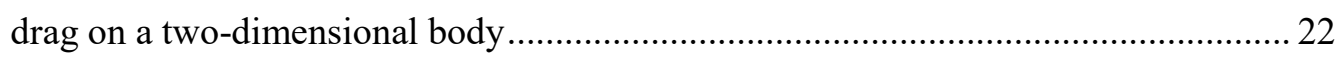

Figure 14: Reconstruction of a 2D instantaneous flow field from Snapshot POD modal analysis 30

Figure 15: Full-scale and scaled non-dimensional ice shapes used for scaling method verification with a) the full-scale and scaled collected ice shapes at $n_{0}=0.3$ and $\mathrm{b}$ ) the full-scale and scaled collected ice shapes at $n_{0}=0.5$. 32

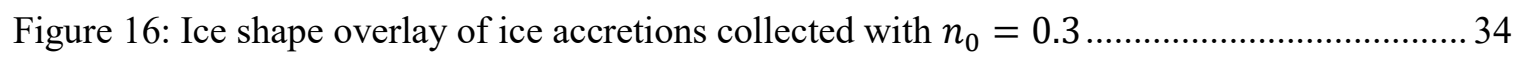

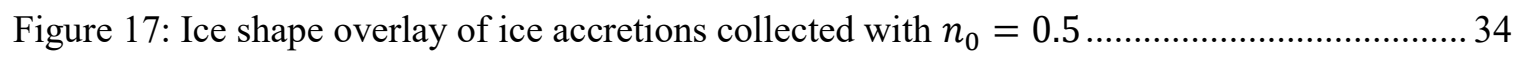


Figure 18: Side, top, and isometric view of $n_{0}=0.3$ full-scale ice shape model

Figure 19: Picture of all seven models used in testing. 35

Figure 20: Close up of $n_{0}=0.3$ full-scale ice shape showing preservation of fine ice features during printing 36

Figure 21: Force-based experimentation in the UD-LSWT schematic 37

Figure 22: Force-Based Experimentation in the UD-LSWT 37

Figure 23: Basic schematic for PIV 39

Figure 24: Illustration of a PIV image pair correlation peak 41

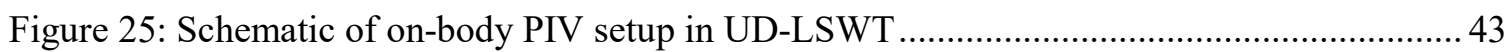

Figure 26: On-body FOV position with respect to the model schematic..................................... 44

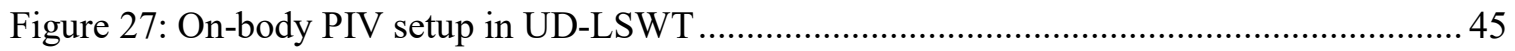

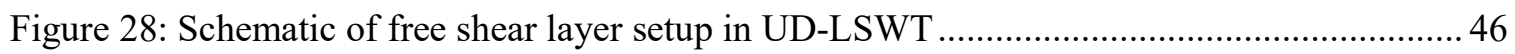

Figure 29: Free Shear Layer FOV position with respect to the model schematic ........................ 47

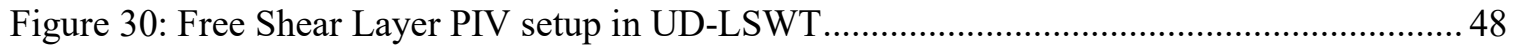

Figure 31: Lift coefficient variation for each ice accretion cases .............................................. 54

Figure 32: Normalized Lift coefficient variation for each ice accretion cases ............................. 55

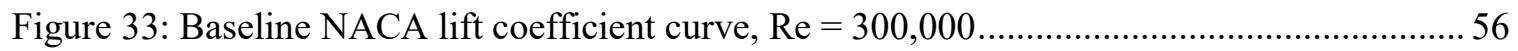

Figure 34: Lift coefficient comparison for $n_{0}=0.3$ ice accretion cases ...................................57

Figure 35: Lift coefficient comparison for $n_{0}=0.5$ ice accretion cases ...................................58

Figure 36: Near wake mean U-velocity profiles for $n_{0}=0.3$ ice accretion cases ....................... 60

Figure 37: Drag coefficient comparison obtained from a) momentum deficit principle b) force-based testing for $n_{0}=0.3$ ice accretion cases

Figure 38: Near wake mean U-velocity profiles $n_{0}=0.5$ ice accretion cases

Figure 39: Drag coefficient comparison obtained from a) momentum deficit principle b) force-based testing for $n_{0}=0.51$ ice accretion cases 66 
Figure 40: Normalized U-velocity contours for $n_{0}=0.3$ ice shapes..................................... 70

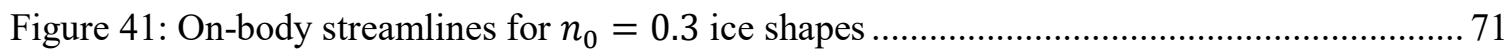

Figure 42: Normalized U-velocity contours for $n_{0}=0.5$ ice shapes........................................ 74

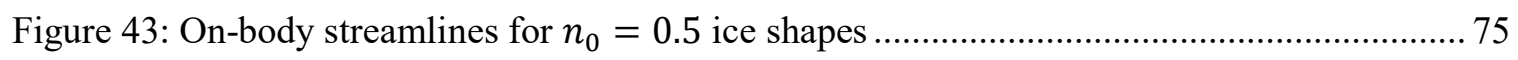

Figure 44: Normalized vorticity contours for $n_{0}=0.3$ ice shapes ....................................... 78

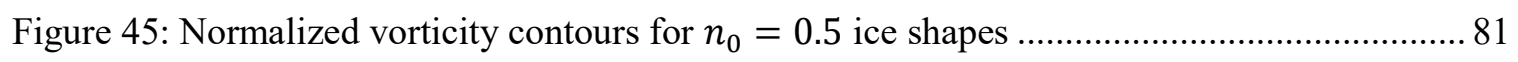

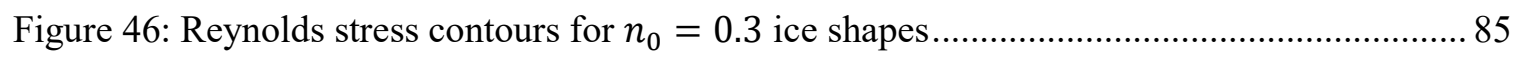

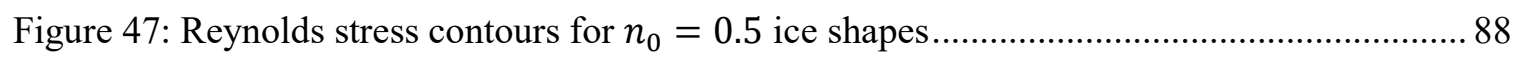

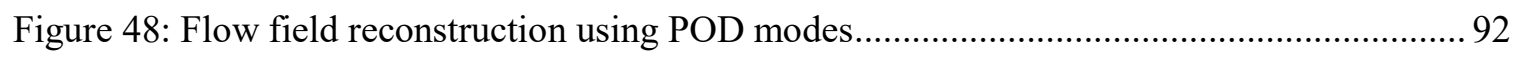

Figure 49: Second and third POD U-velocity correlation modes for $n_{0}=0.3$ ice accretions ...... 94

Figure 50: Second and third POD V-velocity correlation modes for $n_{0}=0.3$ ice accretion cases

Figure 51: Cumulative flow field energy content for first four U-velocity correlation modes for $n_{0}=0.3$ ice accretion cases

Figure 52: Cumulative flow field energy content for first four V-velocity correlation modes for $n_{0}=0.3$ ice accretion cases

Figure 53: Second and third POD U-velocity correlation modes for $n_{0}=0.5$ ice accretions $\ldots . .104$

Figure 54: Second and third POD V-velocity correlation modes for $n_{0}=0.5$ ice accretions $\ldots . .107$

Figure 55: Cumulative flow field energy content for first four U-velocity correlation modes

for $n_{0}=0.5$ ice accretion cases 108

Figure 56: Cumulative flow field energy content for first four V-velocity correlation modes for $n_{0}=0.5$ ice accretion cases 109 


\section{LIST OF TABLES}

Table 1: Similarity Parameter Acceptable Tolerances............................................................. 17

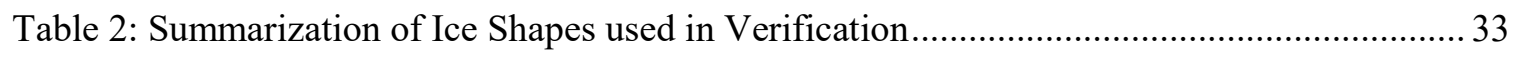

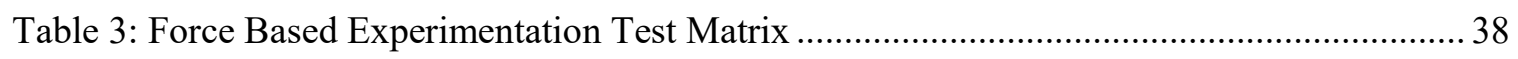

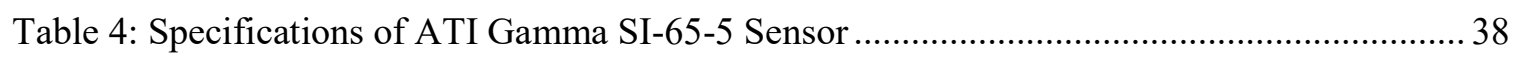

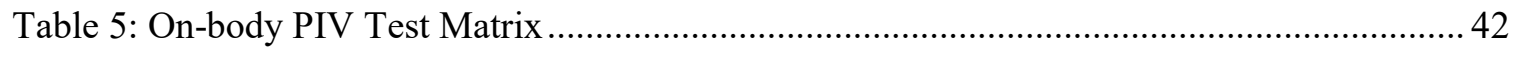

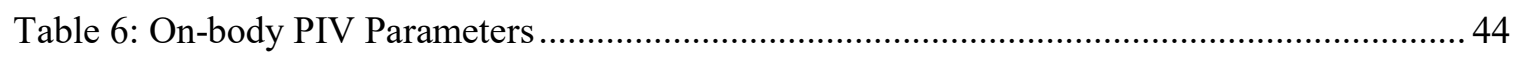

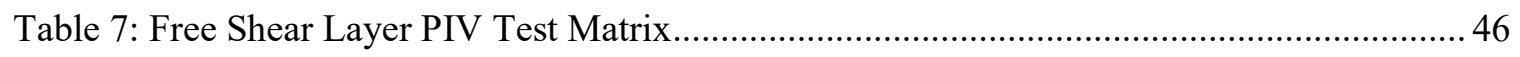

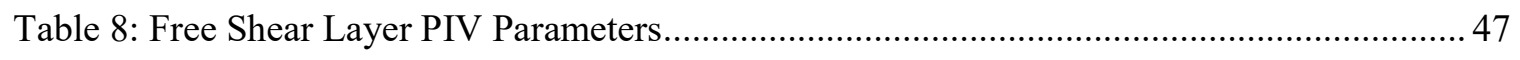

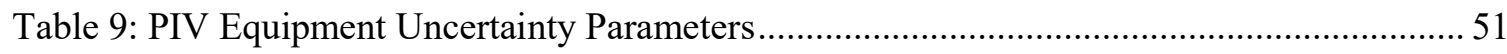




\begin{abstract}
Abbreviations:
AOA - Angle of Attack

CFR - Code of Federal Regulations

DPIV - Digital Particle Image Velocimetry

FAA - Federal Aviation Administration

FOV - Field of view

GRC - Glenn Research Center

IRT - Icing Research Tunnel

ISSI - Innovative Scientific Solutions Incorporated

NACA - National Advisory Committee for Aeronautics

NASA - National Aeronautics and Space Administration

RMS - Root mean squared

SLD - Super-cooled Large Drop

UD-LSWT - University of Dayton Low Speed Wind Tunnel
\end{abstract}

\title{
Common Variables:
}

$A_{C}-$ Accumulation parameter, dimensionless

$A R$ - Aspect ratio, dimensionless

$b$ - Relative heat factor, dimensionless

$c$ - Airfoil chord, $\mathrm{m}$

$C_{d}$ - Two-dimensional coefficient of drag, dimensionless 
$C_{l}-$ Two-dimensional coefficient of lift, dimensionless

$C_{l, \alpha}-$ Two-dimensional theoretical coefficient of lift slope, radians

$C_{L}-$ Three-dimensional coefficient of lift, dimensionless

$C_{L, \alpha}-$ Three-dimensional theoretical coefficient of lift slope, radians

$C_{p}$ - Coefficient of pressure, dimensionless

$h$ - horn height, mm

$K_{0}-$ Modified inertia parameter, dimensionless

L - Length proportional to model chord, cm

$l$ - Characteristic length, $\mathrm{m}$

$L W C$ - Liquid water content of cloud, $\mathrm{g} / \mathrm{m}^{3}$

$M V D$ - Water drop median volume diameter, $\mu \mathrm{m}$

$n_{0}-$ Stagnation freezing fraction, dimensionless

$s$ - Horn location surface length, $\mathrm{mm}$

$s / c$ - Non-dimensional surface length

$t$ - Temperature, ${ }^{\circ} \mathrm{C}$

$W e_{\delta}-$ Weber number based on drop size and water properties, dimensionless

$W e_{L}-$ Weber number based on length, $\mathrm{L}$, and water properties, dimensionless

$\alpha-$ Angle of Attack, degrees

$\beta_{0}-$ Stagnation collection efficiency, dimensionless

$\Phi-$ Drop energy transfer parameter, ${ }^{\circ} \mathrm{C}$

$\theta$ - Horn angle with respect to chord line, degrees

$\theta_{\text {ice }}$ - Air energy transfer parameter, R 
$\tau$ - Accretion time, min

\section{General Subscripts:}

0 - Stagnation point

$s t-$ Static

tot - Total 


\section{CHAPTER I}

\section{INTRODUCTION}

The motivation behind the research performed, an overview of the investigation, and a comprehensive literature review are discussed in this chapter. Throughout the paper, the ice accretion collected on a full-scale wing or model is referenced as the "full-scale ice shape", the ice accretion collected on a scaled model using the recommended ice shape scaling method by Anderson and Tsao [1] is referred to as the "scaled ice shape", and the predicted ice accretion by the LEWICE software [2] is referred to as the "LEWICE ice shape."

\subsection{Motivation}

\subsubsection{SLD Aerodynamic Verification of the Recommended Ice shape Scaling Method}

Aircraft icing is a crucial area of study to ensure operational safety. Due to the safety concern icing poses, all Federal Aviation Administration (FAA) certified aircraft must be operable under the icing conditions specified by the FAA Title 14 of the Code of Federal Regulations (CFR), Part 25, Appendix C envelope [3] and the recently added Appendix O envelope [3], herein referred

to as the Appendix $\mathrm{C}$ envelope and the Appendix $\mathrm{O}$ envelope, respectively. Specialized wind tunnels designed to generate these icing conditions are used to perform FAA certification, along with research into ice formation, ice accretion, and deicing technologies.

Ice accretion investigations are performed in icing research tunnels (IRT) through the simulation of atmospheric icing conditions. This is done by generating an icing "cloud" through injecting chilled water droplets into the wind tunnel flow. The droplets are injected upstream of the test section to allow for sufficient mixing and cooling. Different icing conditions are generated by varying the temperature of the flow, the test section flow velocity, the water droplet median volume diameter (MVD), the liquid water content (LWC), and the ice accretion time, $\tau$. The temperature of the flow is varied using a large heat exchanger and the velocity is altered by changing the drive fan speed. The MVD is the water drop median volume diameter of the icing spray and is varied by 
altering the spray bar air and water pressures [4]. The LWC is the cloud density, given by the amount of water in grams per cubic meter of flow volume. LWC is varied by varying the flow rate through the nozzles [4]. Lastly, the accretion time is the time the model is entrained in the icing conditions. By varying these conditions, aircraft icing is able to be studied across a wide range of desired conditions in the test section.

Due to test section geometric constraints and icing capability limitations, scaling methods regarding both model size and test conditions are used to simulate full-scale icing conditions to study the formation and accretion of ice on aircraft. Through evaluation performed at the NASA Glenn IRT, an icing scaling method was determined by Anderson [5], which is a modified version of the method proposed by Ruff [6]. The method has since been revised and updated by Anderson and Tsao [1]. This updated scaling method is the current recommended icing scaling method. The method is designed to ensure the ice shape produced preserves the non-dimensional reference (fullscale) ice accretion geometry. The full-scale ice accretion refers to an ice accretion collected on a full-scale model, a model with a 1:1 representation of a desired body of interest, at a specified LWC, MVD, temperature, ice accretion time, and flow velocity.

According to Anderson [5], the desired characteristics preserved from the scaling method include: the amount of ice relative to model size, the shape of the ice accreted, and the dimensionless ice accretion limits. Ideally, the aerodynamic penalties due to ice formation are also preserved. Aerodynamic penalties due to ice accretion manifest in combinations of early stall, change in the zero-lift angle of attack, $\alpha_{\mathrm{L}=0}$, and increase in drag. The recommended scaling method from Anderson and Tsao [1] has been geometrically verified in previous literature [5] for the Appendix C envelope and a small range of SLD conditions from $50 \mu \mathrm{m}$ to $190 \mu \mathrm{m}$ [1]. Geometric verification was performed by comparing the non-dimensional cross-sections of an ice shape collected on a full-scale model at specified icing conditions and an ice shape collected on a scaled model at the corresponding scaled icing conditions. This form of verification shows the preservation the desired geometric characteristics which are the amount of ice relative to model 
size, the shape of the ice accreted, the dimensionless ice accretion limits, within allowable tolerances [1][5]. Though the recommended scaling method proposed by Anderson and Tsao [1] has been verified for the Appendix C envelope [5] and for a small range of SLD conditions [1], research showing the preservation of the aerodynamic effects of the accreted ice has yet to be performed.

The FAA recently added icing conditions specified under the Appendix O envelope, which include a large range of SLD conditions including MVD values greater than $500 \mu \mathrm{m}$. Large MVD, especially those above $110 \mu \mathrm{m}$ [1], cause larger icing structures to form further aft on a surface than Appendix $\mathrm{C}$ envelope icing conditions. This is due to larger amounts of water that doesn't immediately freeze on impact with the model surface. These large aft structures are not necessarily preserved by the recommended scaling method and impact the associated aerodynamics. Though the Anderson and Tsao recommended ice shape scaling method was geometrically verified [1], the ice accretions collected on a full-scale and scale model under SLD conditions showed significantly greater geometric variation than those collected under Appendix C conditions. Due to the high geometric similarity found between the ice accretions under Appendix $\mathrm{C}$ conditions, the associated aerodynamics were assumed to be preserved, within allowable tolerances. However, with the significant geometric variations found between the ice shapes formed under SLD conditions, this assumption no longer holds and the aerodynamic verification has yet to be evaluated.

\subsubsection{SLD Aerodynamic Verification of LEWICE}

Experimentation in IRT is an effective way to study aircraft icing and perform FAA icing airworthiness certification. IRT allow for icing to be studied without having to perform multiple flight tests to study icing under the same parameters. The disadvantage of IRT is the geometric constraints on model size due to test section size and the allowable test section blockage. To perform FAA certification and full-scale icing studies, sections of aircraft are typically tested individually for icing certification making the process become time and cost intensive. A swept wing section in the NASA Glenn IRT tunnel is depicted in Figure 1 [7]. As shown, the section in 
truncated chordwise and a false flap is used to simulate similar aerodynamics as a full-length section. The wing section is only $6 \mathrm{ft}$ in height, whereas, most modern commercial aircraft have wingspans greater than $100 \mathrm{ft}$. This highlights the issues associated with performing aircraft icing studies on full-scale aircraft in IRT.

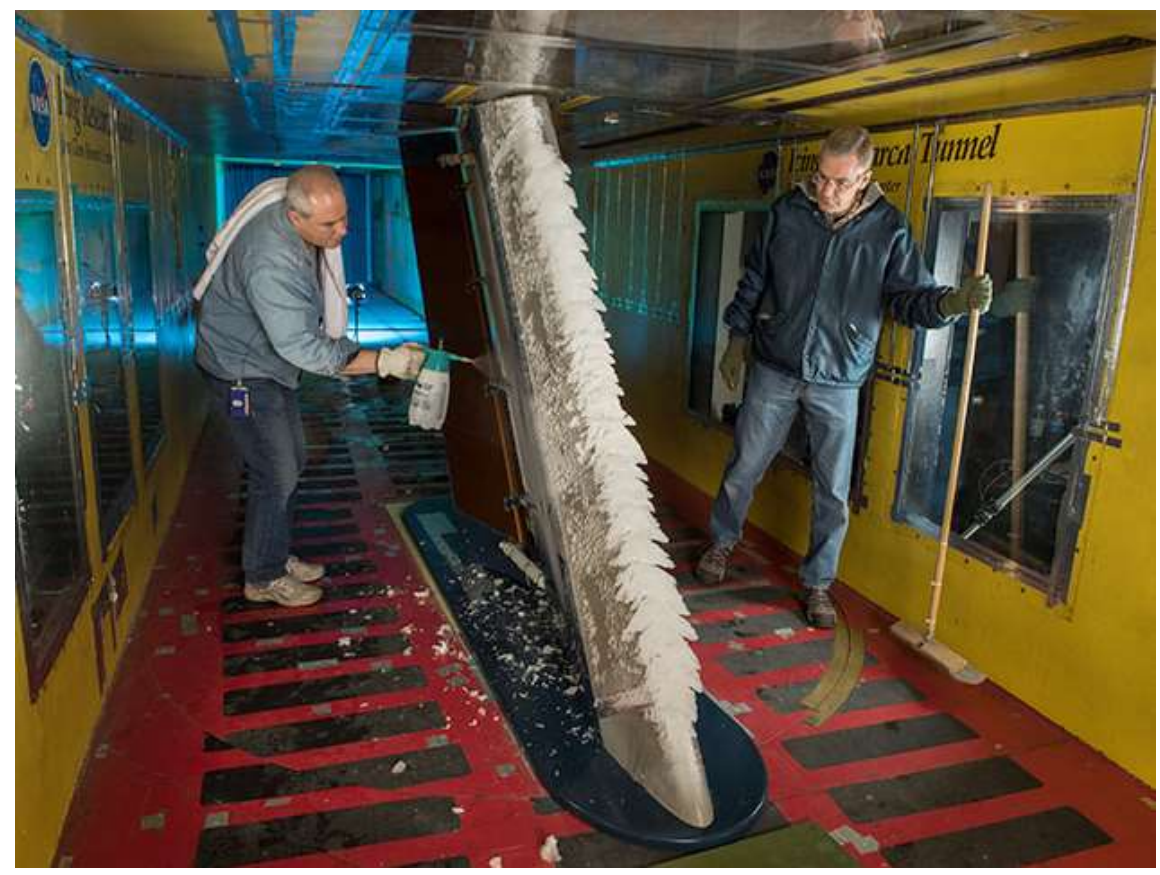

Figure 1: Swept wing leading edge section in NASA Glenn IRT [7]

Due to the geometric constraints of IRT, computational software, such as the LEWICE software [2], are employed to simulate the ice accretion, given the specific icing cloud conditions mentioned prior. The LEWICE software allows a wider range of conditions outside the capabilities of IRT to be studied and for cost effective icing evaluation to be used in the aircraft design process. Computation programs provide the possibility of reduced experimentation for FAA certification of aircraft with respect to icing, if the simulated ice accretion geometry is non-dimensionally similar and the associated aerodynamic penalties are characteristic of the ice accretion collected on a fullscale model or aircraft. 
The verification of the LEWICE software was performed in a similar manner as the recommended scaling method. This was done by evaluating its ability to predict an ice shape that preserves the amount of ice relative to model size, the shape of the ice accreted, and the dimensionless ice accretion limits of a full-scale ice accretion collected under the same conditions. The LEWICE computational software has been verified using base cases and comparing the nondimensional geometry of the LEWICE predicted ice shape to a corresponding ice accretion, within allowable tolerances [2]. Therefore, the LEWICE software has been geometrically verified for certain conditions, including some SLD conditions. Minimal research has been performed providing experimental aerodynamic verification of the LEWICE predicted ice shapes for any conditions.

The LEWICE and scaling method, to this point, have been verified through geometric similitude for SLD conditions. This form of verification shows the appropriate prediction of the amount of ice relative to model size, the shape of the ice accreted, and the dimensionless ice accretion limits, within allowable tolerances, but not of the associated aerodynamic penalties [2].

The preservation of the aerodynamic penalties, is the focus of the research presented in this thesis. The primary objective of this research is to perform low Reynolds number, between 300,000 and 500,000, experimental aerodynamic verification of a NACA 0012 wing with full scale and scaled ice accretions grown under SLD conditions, along with LEWICE predicted ice accretions under the full-scale conditions. The aerodynamic verification was performed through comparison of the coefficient of lift and drag coefficients as a function of angle of attack and comparison of mean and turbulent flow properties in the upper and lower boundary layers between the full-scale, scaled, and LEWICE ice accretions. The near wake is also investigated to determine if the wake profiles are self-similar between the different ice accretions. A modal analysis of the flow in these regions was also performed to evaluate the preservation of coherent structures with dominant energies between the three cases. 


\subsection{Ice Shape Geometry and Nomenclature}

Ice formations in Appendix $\mathrm{C}$ are typically broken into two primary regions, the main ice formation and the feather region ice formation. Figure 2, provided from Anderson and Tsao [1], highlights the two primary ice shape regions. As mentioned by Anderson [5], the primary objective of the ice shape scaling method is to preserve the main region of the leading-edge ice shape as it is the most dominant feature and has the greatest influence on an airfoil's aerodynamics. The LEWICE software only predicts the main ice formation. Though the main region of ice accretions is the most dominant and influential in Appendix C envelope conditions, Anderson and Tsao [1], showed that the large MVD of SLD conditions generate large feather region icing compared to Appendix C conditions, the effects of which cannot be neglected.

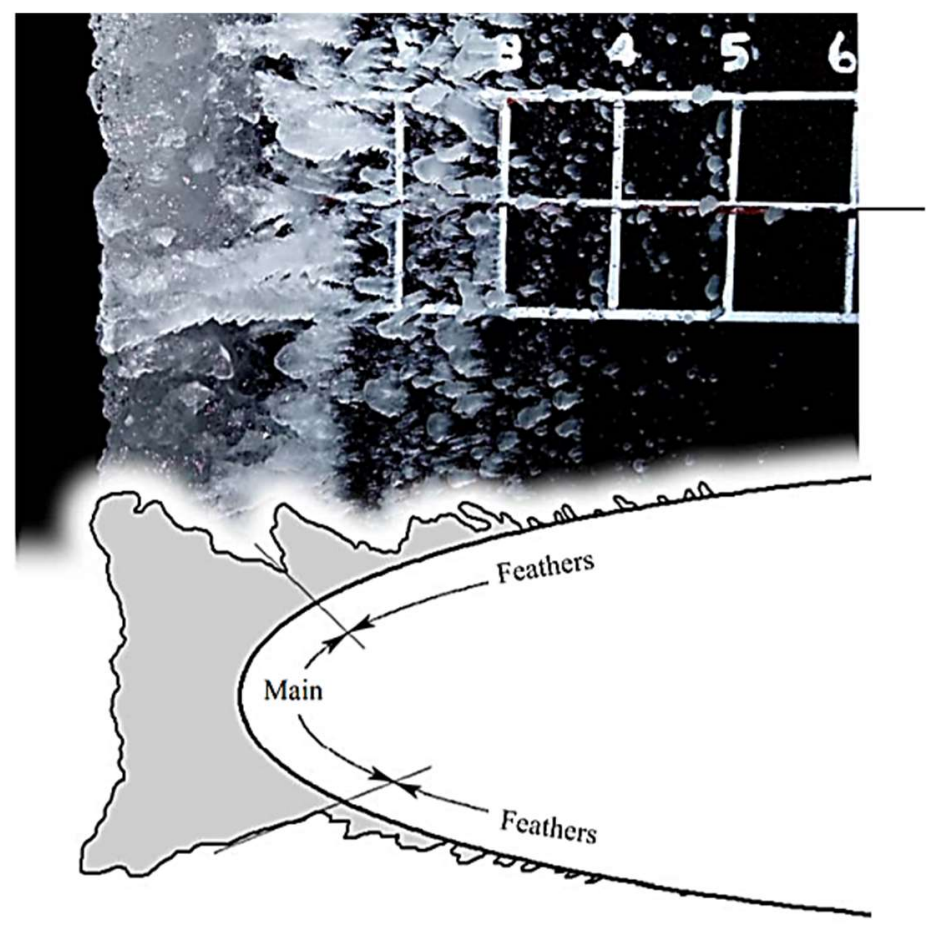

Figure 2: Primary ice shape regions of a given ice accretion in Appendix C conditions [1]

There are four primary representative ice geometries: roughness, horn ice, streamwise ice, and spanwise-ridge ice [8]. Ice shapes may consist of features characteristic of more than one of these ice geometries, but are typically separated into these classifications. Roughness ice is highly three-dimensional and is characterized by a main region consisting of a smooth-zone and a rough 
zone, as shown in Figure 3 [9]. Roughness ice occurs during the initial phase of ice accretion, prior to the accretion of a more dominant ice shape, such as streamwise or horn ice [8]. Roughness ice mostly affects the local boundary layer.

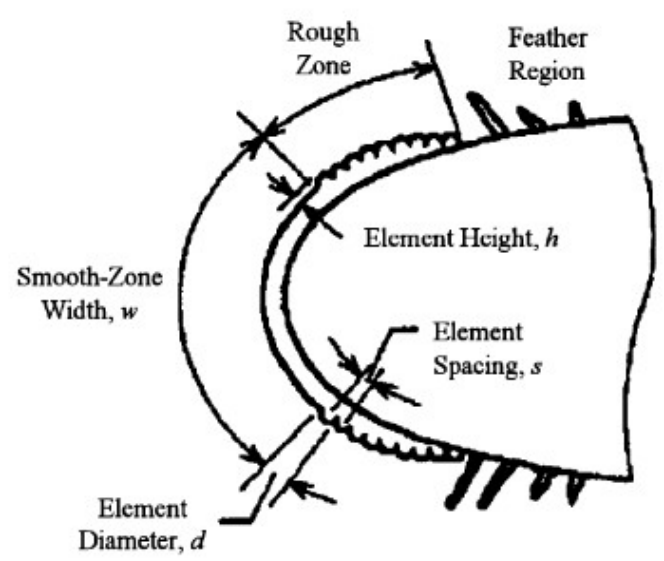

Figure 3: Roughness ice shape geometric schematic [9]

Horn ice geometry can feature either a single or a double horn. Figure 4 presents a simplified schematic of a double horn ice shape with only the main ice accretion region being shown [8]. Double horn ice accretions are typically generated at lower, more neutral angles of attack. The schematic in Figure 4, provided by Bragg et al. [8], outlines the geometric parameters used to characterize a horn ice accretion. The horn shape is characterized by three geometric parameters: the angle it makes with respect to the chord line, $\theta$, the horn height, $h$, and the nondimensional surface length, $s / c$, where $s$ is the surface length location of the horn shape and $c$ is the chord length [8]. These parameters all have a large effect on the aerodynamic penalties associated with horn ice accretions. The horn ice geometry is strongly influential on the airfoil aerodynamics, generating primarily two-dimensional flow separation. Horn ice is typically formed under mixed ice conditions at intermediate flow temperatures. Larger MVD or high LWC can also generate this is shape, therefore, SLD conditions commonly result in a horn ice shape due to the large MVD values. 


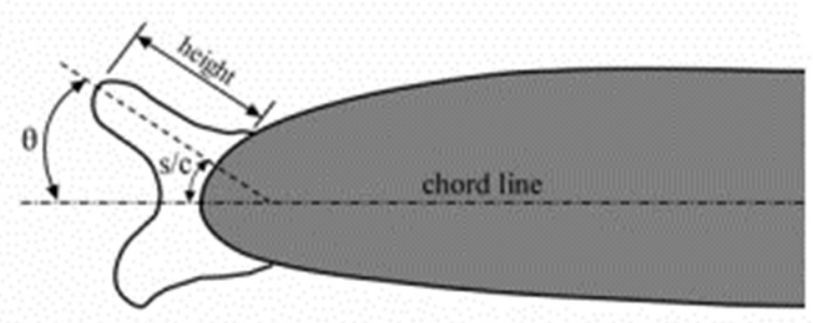

Figure 4: Horn ice shape geometric schematic [8]

Streamwise ice is characterized by a main region ice accretion that follows the contour of the surface [8]. A qualitative sketch highlighting the main region of a streamwise ice shape from Blumenthal [10] is presented in Figure 5. Like the horn ice shape, the aerodynamics of a streamwise ice shape are dominated by two-dimensional flow separation. However, it is significantly less influential on the overall flow field aerodynamics. Streamwise ice is typically accreted under rime ice conditions, where the airflow temperature is low causing impinging water droplets to completely freeze on impact [8].

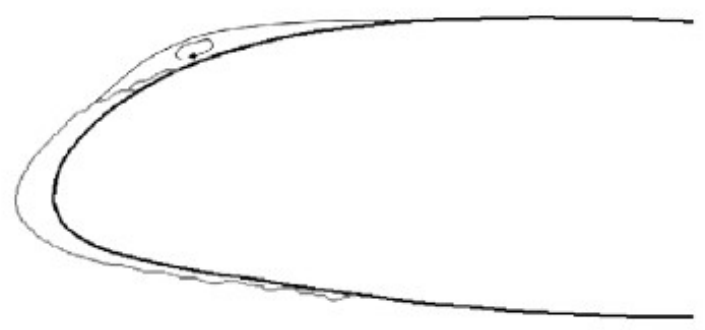

Figure 5: Qualitative sketch of streamwise ice [10]

The last category of ice geometry, the spanwise-ridge ice, is highly influential on the flow field aerodynamics. Spanwise-ridge ice forms further downstream on an airfoil than the other ice geometries and has characteristics of a flow obstacle, greatly influencing the aerodynamics [8]. Spanwise-ridge ice is typically generated when deicing is present, but not operating at a point of $100 \%$ evaporation [8]. This causes water to run further downstream to where the deicing technology is not applied and freeze, forming a ridge/lip like structure. Figure 6 shows a schematic of a simulated quarter round span-wise-ridge ice shape [11]. 


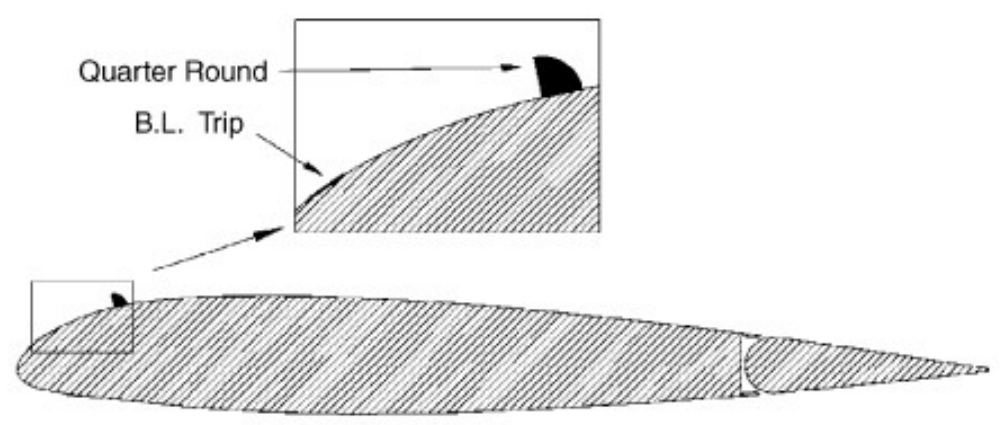

Figure 6: Simulated quarter-round spanwise-ridge ice [11]

The aerodynamic evaluation performed in this paper focuses on horn shaped ice accretions due to their complex geometries and high aerodynamic sensitivity to geometric differences in the horn. Horn ice shapes are common for SLD conditions for which deicing technologies are not being used. The initial geometric verification of the ice shape scaling method by Anderson [5] and of the LEWICE software for SLD conditions was performed though evaluation of preserving horn ice accretions [1][2]. The ice shapes in the current investigation were all collected at an angle of attack of $0^{\circ}$ and have a double horn ice accretion.

\subsection{Horn Iced Airfoil Aerodynamics}

There have been multiple studies performed to investigate the aerodynamics associated with airfoil icing. This includes studies performed to quantify the aerodynamic penalties in regard to lift and drag, and to study the sensitivities of the preceding changes in the ice shape geometry and roughness [8][12][13][14][15]. Research into the aerodynamics of horn ice accretions indicates that the surface roughness and small variations in the horn ice accretions that occur along the length of the leading edge have a marginal impact on the associated aerodynamics [8].

The aerodynamics associated with horn ice accretions is dominated by the separation bubble that forms aft of the horn due to the adverse pressure gradient caused by the significant surface geometry discontinuity [8]. This separation bubble shares characteristics with laminar separation bubbles that may form on clean airfoils or on a surface when an adverse pressure gradient is present. Many studies have been performed to understand the aerodynamics associated with 
laminar separation bubble; therefore, a brief description of laminar separation bubble flow dynamics, adapted from Roberts [16], is provided. A schematic of an instantaneous laminar separation bubble flow field caused by an induced adverse pressure gradient on the surface of a flat plate is shown in Figure 7 [16].

As shown in Figure 7, the coefficient of pressure, $C_{p}$, plateaus between points $S$ and $T$ due to the static pressure in the separation bubble being highly constant in this region. The separation bubble causes the formation of a shear layer. The shear layer proceeds to transition to turbulent flow at point $T$. Once the shear layer becomes turbulent, the magnitude of the reverse flow increases and vorticial flow forms in the separation bubble [8]. This causes the pressure in the separation bubble to become highly unsteady and possible vortex shedding to occur. The freestream flow energizes the shear layer causing the turbulent transition at point $\mathrm{T}$ and pressure recovery occurs leading to flow reattachment at point $\mathrm{R}$.

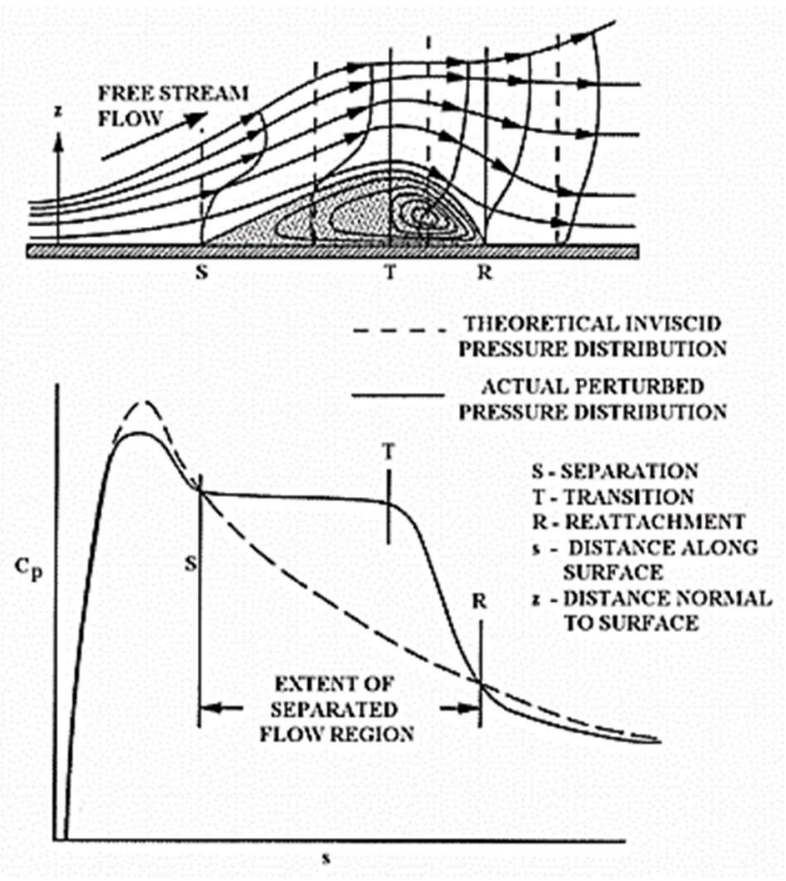

Figure 7: Instantaneous laminar separation bubble flow field schematic of a flat plate with an induced adverse pressure gradient at point $S$, with corresponding pressure distribution [8] 
The similarities between the separation bubble formed due to a horn ice shape and a laminar separation bubble on a clean airfoil were reiterated by Gurbacki and Bragg [12]. A schematic of the flow phenomena of the separation bubble caused by a single horn ice shape can be found in Figure 8, provided by Gurbacki and Bragg [12]. Figure 8 highlights the separation location of the flow at the tip of the horn ice formation.

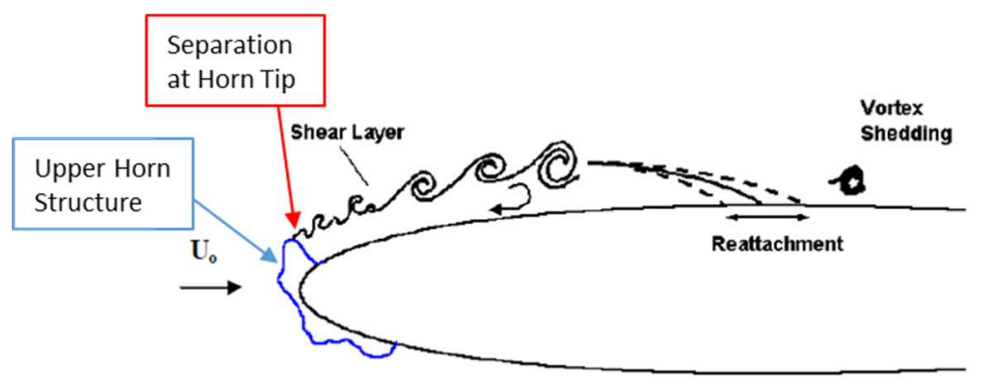

Figure 8: Upper surface separation bubble on a NACA 0012 schematic [12]

The length of the separation bubble, which is noted as the distance between the point of separation at the horn ice tip, as shown in Figure 8, and the reattachment location, varies with angle of attack. Figure 9 shows how the size and length of the separation bubbles on the upper and lower surfaces of an airfoil with a double horn ice shape changes with increasing angle of attack. The separation streamlines in Figure 9, were determined experimentally and collected on a NACA 0012 with a simulated horn ice shape at a Reynolds number of $1.5 \times 10^{6}[17]$.

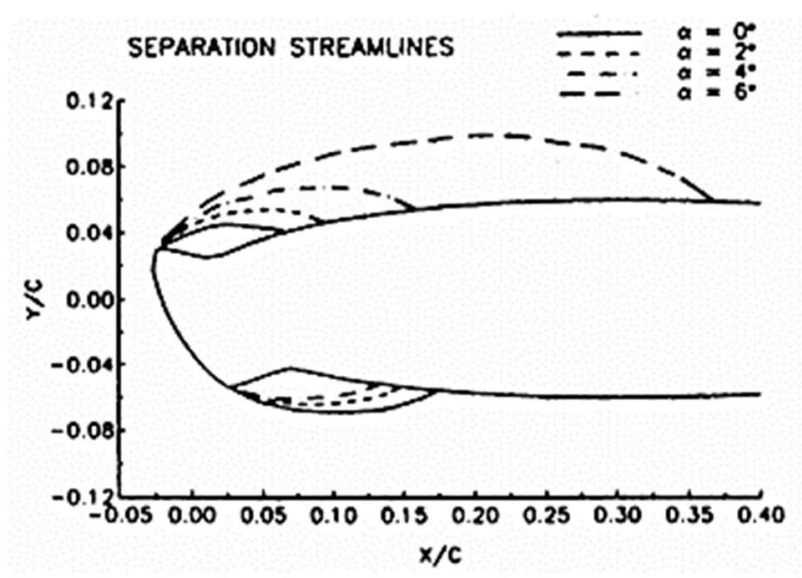

Figure 9: Separation streamlines on a NACA 0012 airfoil with a simulated horn accretion [17] 
Bragg et al. [8] determined the separation point at the horn tip was consistent. Therefore, the dominant characteristics effecting the separation region, and in turn the aerodynamic performance of the airfoil, were the horn height and location. Therefore, the $s / c, \theta$, and $h$, as specified in Figure 4, are the most important horn ice geometric parameters to preserve during scaling or simulation, as they have the largest influence on the preservation of the associated aerodynamics. Multiple studies have been performed showing high aerodynamic sensitivity to the

height and location of the horn ice [12][14][15][16]. The greater $\theta$ and $s / c$, the greater the aerodynamic penalty with respect to increase in drag and earlier stall. The same relationship was found for the horn ice height, $h$, with the exception of when the ice shape was located near the leading edge of the airfoil, i.e. a small $\theta$ and an $s / \mathrm{c}$ close to zero. The iced airfoil aerodynamics were also found to be insensitive to Reynolds number [13][14].

\subsection{Ice Shape Scaling Method and Previous SLD Verification}

As mentioned, due to test section geometric constraints and icing limitations, scaling methods to scale both the model size and icing test parameters are employed to study the formation and accretion of ice on aircraft surfaces, especially on the wing leading edge. These methods are designed to ensure the ice shape produced, specifically the main leading-edge ice formation, is nondimensionally characteristic of the ice accretion formed on a full-scale model. Scaling methods developed by Ruff [6] and Olsen [18] were evaluated in Manual of Scaling Methods by Anderson [5]. The scaling method determined from Anderson's evaluation [5] of methods proposed by Ruff [6] and Olsen [18], has since been revised and updated. The current recommended ice shape scaling method was evaluated and determined by Anderson and Tsao [1] at the NASA Glenn IRT. It is based on the initial method provided by Anderson [5], but allows for more flexibility in scaling the physical test conditions.

The recommended ice shape scaling method utilizes a modified version of the method proposed by Ruff [6], in which the modified inertia parameter, $K_{0}$, the accumulation parameter, $A_{c}$, the stagnation freezing fraction, $n_{0}$, and the Weber number based on the model length and the water 
properties, $W e_{L}$, are matched between the scale and full-scale (reference). Through the similarity parameters, the wind tunnel conditions required to generate a characteristic ice shape are determined. The wind tunnel flow parameters, mentioned previously, include the temperature of the flow, the test section freestream velocity, MVD, LWC, and the ice accretion time, $\tau$.

$A_{c}$ and $K_{0}$ relate to the droplet trajectory in the flow. $A_{c}$ is used to ensure the scale and reference ice shapes are of similar non-dimensional size. It is essentially equal to the thickness of the ice accretion that would form if all droplets in the path of the model impinged on the leading edge and froze normalized by the model characteristic length, typically the leading-edge nose diameter of curvature. Since some of the water droplets initially in the path of the model will divert due to the airflow around the model, the droplet inertia is considered. $K_{0}$ accounts for the droplet inertia and trajectory. Shown by Langmuir and Blodgett [19], the stagnation collection efficiency, $\beta_{0}$, is a function of only $K_{0}$. Therefore, $\beta_{0}$ and $K_{0}$ can be used interchangeably as a similarity parameter.

The freezing fraction, $n$, is the ratio of the amount of water that freezes at a given location to the amount of water that reaches the location [1]. The stagnation freezing fraction, $n_{0}$, is the freezing fraction at the stagnation point. $n_{0}$ is a function of the drop energy transfer parameter, $\Phi$, the air energy transfer parameter, $\theta_{i c e}$, and the relative heat factor, $b$, therefore accounting for the matching of multiple ice accretion characteristics. This similarity parameter accounts for the amount of water that does not freeze immediately on initial contact and disperses to a different location. Higher freezing fractions generate horn icing with lower horn angles and lower s/c positioning due to more ice freezing on impact near the stagnation point.

The Weber number is used to scale the test section freestream velocity. The Weber number based on the model length and the water properties, $W e_{L}$, is held constant between the scaled and full-scale icing cases. The Weber number is the ratio of a fluids inertia to surface tension as given by Equation 1, where $\rho$ is the density of the fluid, $v$ is the freestream velocity of the fluid, $l$ is the characteristic length (chord in the case of an airfoil), and $\sigma$ is the surface tension of the fluid. By 
keeping $W e_{L}$ constant, the scale to reference velocity ratio becomes equivalent to the square root of the reference to scale size ratio, Equation 2.

$$
\begin{gathered}
W e=\frac{\rho v^{2} l}{\sigma} \\
\frac{v_{\text {scaled }}}{v_{\text {full-scale }}}=\sqrt{\frac{l_{\text {full-scale }}}{l_{\text {scale }}}}
\end{gathered}
$$

It is important to note that when the velocity is scaled using the Weber number, the Reynolds numbers of the scaled and full-scale models are not equivalent during the ice accretion experimentation. This coupled with the differences in the ice accretions provided the motivation for aerodynamic verification of ice accretions on the full-scale and scaled model through isolated testing of each at the same Reynolds Number.

Using the calculated similarity parameters, the physical test parameters, including the ice accretion time, the MVD, the flow freestream velocity, the temperature, and the LWC are determined and set for the scaled model. A brief discussion of the steps in determining the physical test parameters is adapted from Anderson and Tsao [1]. The velocity based on the Weber number using equation 2 is the first step in the recommended scaling process. The scaled velocity is then used along with $\beta_{0}$ to determine the MVD. The scaled LWC is chosen based off of convenience for test operations. The scaled static temperature is then determined based on matching $n_{0}$ and allows for the total temperature to be calculated. If the total temperature is greater than $-2^{\circ} \mathrm{C}$, a new LWC is chosen and the static temperature recalculated. The final step in the scaling method is to determine the scaled ice accretion time. If $\beta_{0}$ is held perfectly constant between the scaled and fullscale case, then the scaled ice accretion time is determined from $A_{c}$. However, if this not the case, the scaled ice accretion time is determined based on matching the product of $\beta_{0}$ and $A_{c}$.

The recommended method of ice shape scaling was verified for Appendix $\mathrm{C}$ envelope conditions by comparing the non-dimensional characteristic $2-\mathrm{D}$ ice shape accreted on a $91.44 \mathrm{~cm}$ 
chord NACA 0012 airfoil to the non-dimensional 2-D ice shape accreted on a $53.34 \mathrm{~cm}$ chord NACA 0012 airfoil. The $91.44 \mathrm{~cm}$ airfoil was used as the full-scale model and the $53.34 \mathrm{~cm}$ airfoil was used as the scaled model. Figure 10 presents a comparison for Appendix $\mathrm{C}$ conditions. The Appendix C condition comparison is provided from Anderson [5].

Anderson and Tsao's recommended scaling method [1] was verified geometrically, within allowable tolerance, for limited SLD conditions using ice accretions formed on a $183 \mathrm{~cm}$ NACA 0012 airfoil and a $27 \mathrm{~cm}$ chord NACA 0012 airfoil [1]. The $183 \mathrm{~cm}$ chord airfoil was used as the full-scale and the $27 \mathrm{~cm}$ chord airfoil was used as the scaled model. Figure 11 presents a comparison for SLD conditions. The SLD condition comparison is provided from Anderson and Tsao [1].

The ice shapes in Figures 10 and 11 are tracings collected at the midspan of the NACA 0012 models in the NASA Glenn IRT. This method does not capture the spanwise variation of the ice shape, primarily in the feather region, or the surface roughness. Though these are simplified representations of the ice accretion, they were consistently collected at the midspan and still represent the geometric variation between the full-scale and scaled ice shapes. The NASA Glenn IRT is currently developing techniques to gather three-dimensional scans of the ice shapes.

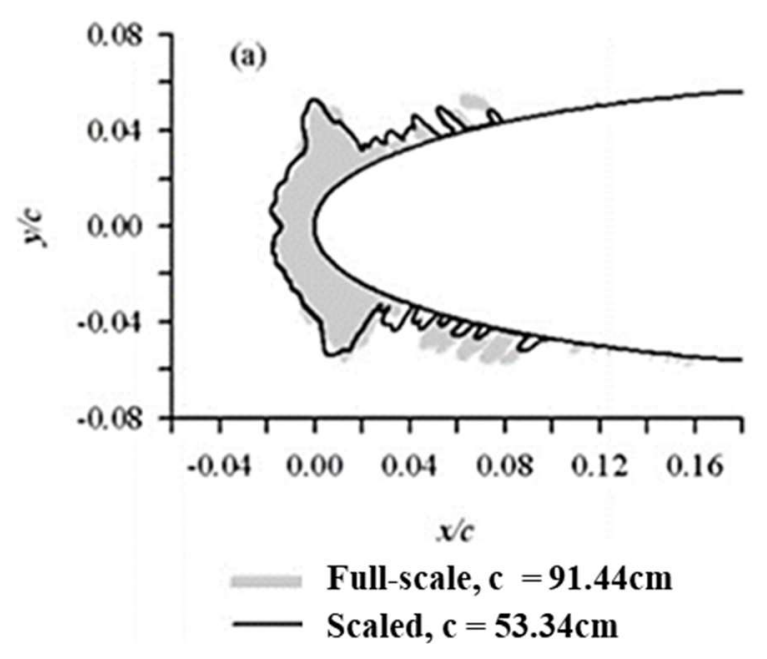

Figure 10: Scaled and full-scale ice accretion used in the verification for Appendix C conditions [5] 


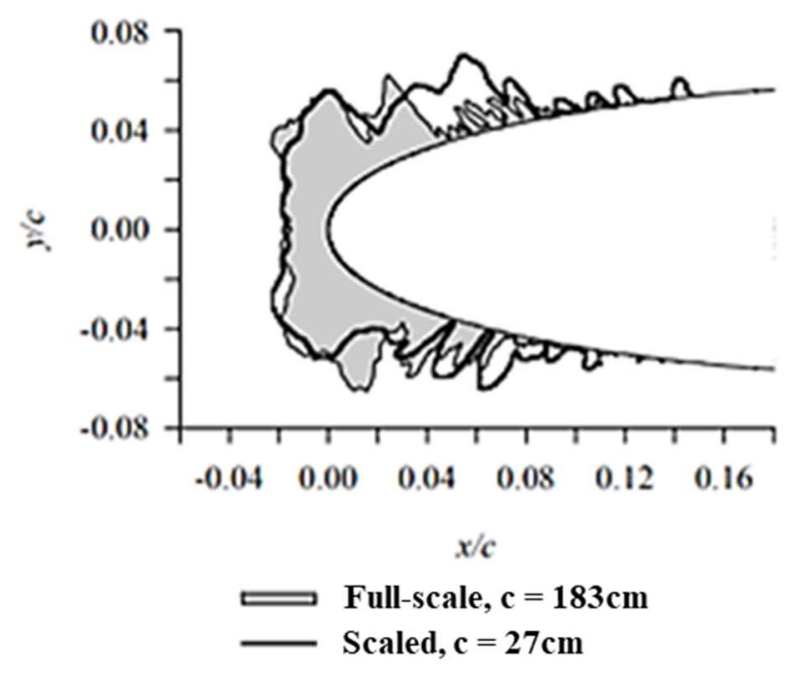

Figure 11: Scaled and full-scale ice accretion used in the verification for SLD conditions [1]

The scaling method is clearly shown to be highly effective in reproducing the ice shape under Appendix C conditions, but not to the same degree under the SLD conditions. Anderson and Tsao [1] attributed this to the larger feather region accretions that occur under SLD conditions due to the large MVD. Both the scaled and full-scale ice shapes in Figure 11, have large feather region ice accretions, especially on the upper surface of the scale ice shape. It was noted that when $\beta_{0}$ was perfectly matched between scaled and full-scale testing, the feather region accretions were best matched [1], but still had deviation. This is shown by the ice shapes in Figure 11 for which $\beta_{0}$ was matched between the full-scale and scaled ice shapes.

Due to uncertainties in the test conditions and the inability to exactly match the test parameters between the reference model and the scaled model, Anderson [5] discusses the acceptable tolerances for the different similarity parameters. Anderson [20] studied the acceptable tolerances, which are summarized in Table 1 . If $\beta_{0}$ cannot be matched exactly, it is recommended that the product of $A_{C}$ and $\beta_{0}$ be held within a tolerance of $10 \%$ between reference and scale. It is important to note that these tolerances were evaluated using Appendix C conditions. SLD 
conditions will most likely require tighter tolerances, since there is larger variation between the ice shapes, as shown in Figure 11.

Table 1: Similarity Parameter Acceptable Tolerances [20]

\begin{tabular}{|c|c|}
\hline Similarity Parameter & Tolerance \\
\hline$A_{c}$ & $10 \%$ \\
\hline$n_{0}$ & $10 \%$ \\
\hline$B_{0} A_{c}$ & $10 \%$ \\
\hline$W e_{L}$ & $15 \%$ \\
\hline
\end{tabular}

\subsection{LEWICE}

The LEWICE software is an ice accretion predictive software based on an analytical model of the thermodynamics involved in icing. Since the flow dynamics in the feather region is not well understood, LEWICE only focuses on the prediction of frontal ice shapes. The model utilizes a significant number of atmospheric or IRT conditions to predict the ice shape formation on numerous aircraft surfaces and can even include the effects of different deicing methods. The goal of the software is to accurately predict the location, size, and shape of an ice accretion based on surface geometry and icing conditions [2]. LEWICE provides a method for studying multiple aircraft icing situations without the necessity for extensive experimentation in an IRT and can predict two-dimensional or three-dimensional ice accretions.

The four modules used in LEWICE to predict an ice formation include the flow field calculation, the particle trajectory and impingement calculation, the thermodynamic and ice growth calculation, and the modification of the current geometry by adding the ice growth to it [2]. These models use multiple inputs outlined in User's Manual for LEWICE Version 3.2. The icing cloud (meteorological) and atmospheric conditions are used as inputs to the LEWICE software for prediction. These parameters include the geometry of the surface, the temperature, the pressure, the flow velocity, the $L W C$, the $M V D$, and the relative humidity [1]. Using these parameters along with other specifications, the LEWICE software predicts an ice formation. Examples of LEWICE input 
files can be found in Appendix A of this paper, which includes the input files used to generate the LEWICE structures used in this investigation.

The LEWICE model has been verified through non-dimensional geometric comparison under multiple icing conditions. Verification specifically performed for SLD conditions was performed by Wright [21] and showed LEWICE was as accurate at predicting SLD ice formations as Appendix C formations, verified by Wright [2], however, the verification was for a small range of SLD conditions. The investigation also showed the LEWICE software was able to predict the frontal ice shape in SLD conditions to the same accuracy as the scaling method is able to simulate the frontal ice shape. As for aerodynamically, the LEWICE software has been validated using a Navier-Stokes flow solver [22] and compared to the coefficients of lift and drag of a LEWICE ice accretion and one collected in the NASA GRC IRT. The Navier-Stokes solver showed good similarity between the coefficient of lift and drag of the multiple ice shape pairings tested; however, no experimental research has been produced to aerodynamically verify the LEWICE software.

\subsection{Overarching Research Objectives}

The objective of this research is to provide an initial low Reynolds number aerodynamic verification the ice accretions generated by the LEWICE software and ice shape scaling method under SLD conditions by comparing the surface and near wake aerodynamics and the associated lift and drag coefficients with that of the full-scale ice accretion under two different stagnation freezing fractions, $n_{0}$. The definition of what constitutes a full-scale model is annotated in Figure 10 and 11 . The verification will focus on two-dimensional ice geometries collected through tracing the ice shape at the model midspan for the scale and full-scale models in the NASA IRT and predicted through the two-dimensional prediction condition of the LEWICE software.

As discussed, two-dimensional tracings are a simplified modeling of the collected ice shapes, as they do not include the ice surface roughness or the spanwise variation of the ice shape, especially with respect to the feather region. Though these features may not be included, the 
verification performed in this thesis will still express whether the recommended scaling method and LEWICE software are able to produce an ice shape that preserves the aerodynamic effect of the full-scale ice accretion under SLD conditions. The research performed will also determine regions of interest with respect to the ice accretion geometry, initiate the discussion of what is considered tolerable aerodynamic variation from the full-scale, and set a procedure for evaluating the aerodynamic verification of ice shape scaling methods and ice shape predictive software.

Aerodynamic verification was done by quantifying the self-similarity of aerodynamic coefficients and the mean and fluctuating flow properties between the reference (full-scale) model, the scaled model, and LEWICE prediction, using force-based testing and an optical flow diagnostic technique, such as Particle Image Velocimetry (PIV). The experimental objectives were to

1. Perform static force-based experimentation to determine the lift and drag coefficients as a function of angle of attack at different Reynolds numbers for all three ice accretion cases.

2. Perform Particle Image Velocimetry (PIV) around the ice accretions and in the near wake for all three cases to quantify the changes in the mean and fluctuating flow properties for the three ice accretion cases.

The flow field analysis of these regions allowed for the comparison of quantities, such as the mean velocity component, the vorticity, and the Reynolds stress. These flow properties allow for the study of the separation bubbles on the upper and lower surface due to the horn ice along the airfoil's surface. The streamwise component analysis of the near wake free shear layer allowed for a second evaluation of the coefficient of drag though momentum deficit principle.

Due to the complexity of the flow, the important physical features, such as coherent structures, were extracted by performing Proper Orthogonal Decomposition (POD) to the on-body and near wake flow fields. POD is a modal decomposition process that allows for the modes and the energy present in each mode of the flows to be evaluated and compared. Altogether, this experimental analysis allowed for the aerodynamic verification of the ice accretion prediction by 
the LEWICE software and ice accretion obtained from the ice shape scaling method under SLD conditions. 


\section{CHAPTER II}

\section{THEORETICAL BACKGROUND}

In order to evaluate the aerodynamics associated with the ice accretions, multiple aerodynamic coefficients and flow parameters were assessed. Each of the flow field parameters are defined mathematically and by the qualitative importance.

\subsection{Flow Field Analysis}

The flow around iced airfoils is inherently turbulent. Therefore, tools and techniques used to analyze turbulent flows are required. Reynolds decomposition breaks a turbulent flow instantaneous velocity into the sum of the mean and the fluctuating velocity, allowing for the mean and fluctuating velocity components of the turbulent flow to be assessed. Reynolds decomposition is mathematically described in Equation 3 and depicted in Figure 12 from Gunasekaran [23]. The bar in Equation 3 represents time averaged, or mean quantities, and the prime denotation represents the fluctuating component. The subscript $i$ denotes the flow field dimension and is equal to 1 or 2 for two-dimensional flows and equal to 1, 2, or 3 for three-dimensional flows.

$$
\tilde{u}=\overline{U_{i}}+u_{i}^{\prime}
$$

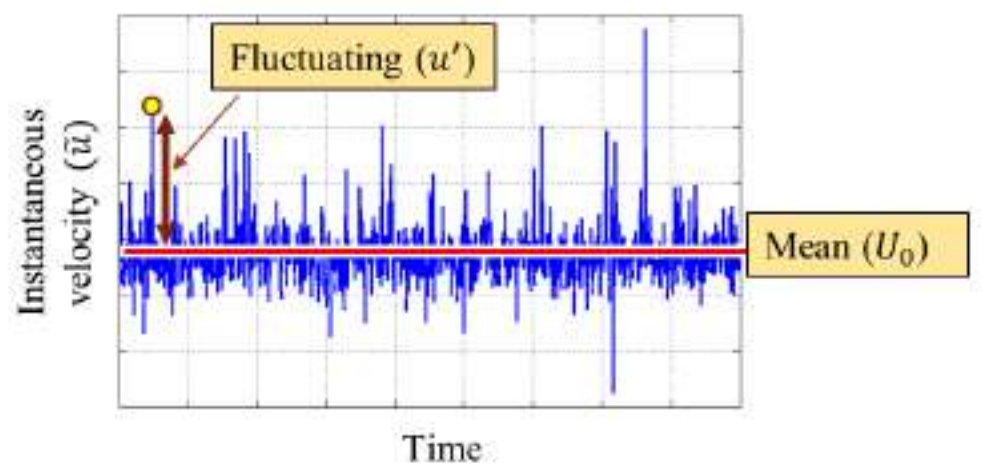

Figure 12: Reynolds decomposition of a turbulent flow [23] 
To properly analyze the turbulent flow field, its corresponding mean and fluctuating properties must be investigated. The mathematical formulations used to determine the momentum deficit, the Reynolds stress, and vorticity in the flow are discussed in the following subsections.

\subsubsection{Near Wake Momentum Deficit}

The schematic found in Figure 13 provides a Cartesian reference plane for the flow around a twodimensional body, expressing the mean streamwise velocity at the inflow, station 1 , and the outflow, station 2 [24].
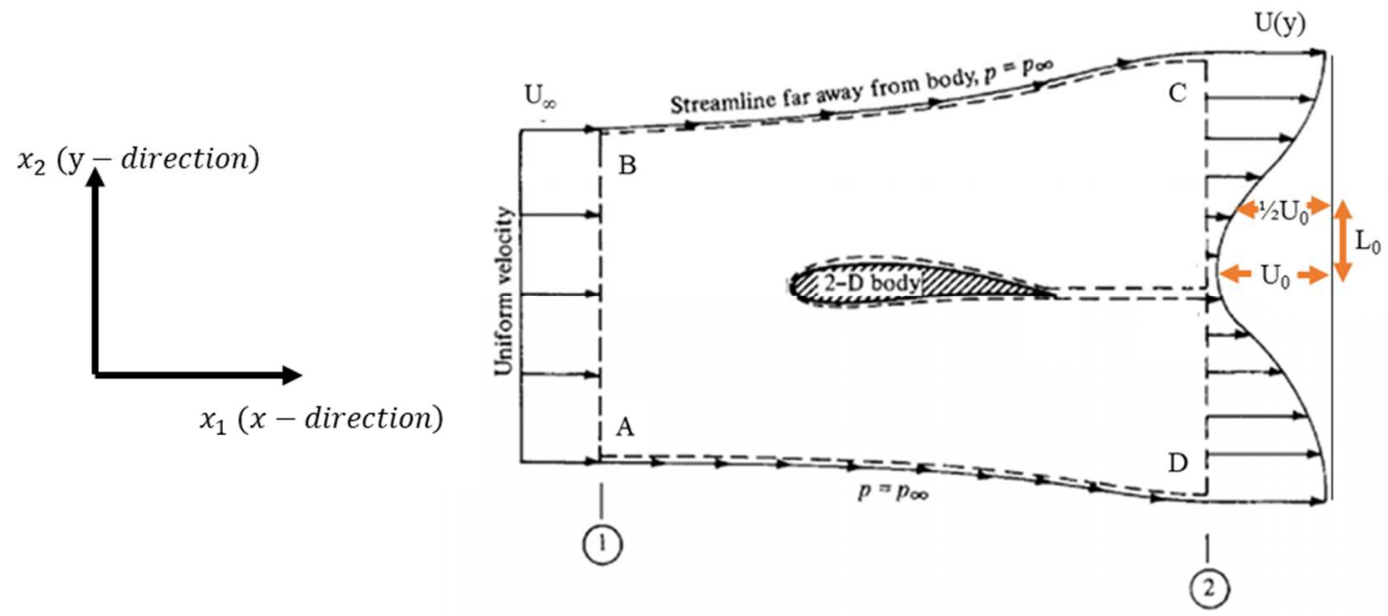

Figure 13: Flow around a body in a two-dimensional plane, control volume for obtaining drag on a two-dimensional body [24]

The streamwise near wake velocity was used to determine the two-dimensional drag of the airfoils with different ice shapes to corroborate the drag results from the force-based testing. The drag is calculated based on the conservation of momentum provided by Newton's second law. By accounting for the loss of momentum in the streamwise flow, the drag on the body can be accurately calculated. This method of determining drag is known as momentum deficit principle.

The mathematical approach to calculate drag using momentum deficit is provided in the following and is adapted from Anderson [24]. Figure 13 is used to specify the control volume and other parameters used in the determination of the drag. Figure 13 shows an example velocity profile $U(y)$ behind an airfoil in a uniform freestream flow, where $U_{\infty}$ is the velocity of the streamwise 
freestream flow, $U_{0}$ is the amplitude of the wake velocity profile, and $L_{0}$ is the half wake width. $L_{0}$ is equivalent to half of the wake amplitude and given in Equation 4 [23].

$$
L_{0}=\frac{U_{0}}{2}
$$

Assuming the flow is inviscid and one-dimensional, the three-dimensional Euler momentum equation, Equations 5 through 7, can be simplified to Equation 28. The simplified equation is set equal to a placeholder variable $M$, which represents the momentum loss in the freestream flow. The terms on the right side of the Euler momentum equation are the pressure and body-force terms, respectively.

$$
\begin{aligned}
X-\text { Direction } & \rightarrow \oiiint \frac{\partial\left(\rho u_{1}\right)}{\partial t} d V+\oiint(\rho \bar{V} \cdot d A) u_{1} \\
& =-\oiiint \frac{\partial p_{1}}{\partial x_{1}} d V+\oiint \rho f_{1} d V \\
Y-\text { Direction } & \rightarrow \oiiint \frac{\partial\left(\rho u_{2}\right)}{\partial t} d V+\oiint(\rho \bar{V} \cdot d A) u_{2} \\
& =-\oiiint \frac{\partial p_{1}}{\partial x_{2}} d V+\oiiint \rho f_{2} d V \\
Z-\text { Direction } & \rightarrow \oiiint \frac{\partial\left(\rho u_{3}\right)}{\partial t} d V+\oiint(\rho \bar{V} \cdot d A) u_{3} \\
& =-\oiiint \frac{\partial p_{3}}{\partial x_{3}} d V+\oiiint \rho f_{3} d V
\end{aligned}
$$

Simplified Euler momentum equation using 1D and inviscid assumption,

$$
\oiiint \frac{\partial\left(\rho u_{1}\right)}{\partial t} d V+\oiint(\rho \bar{V} \cdot d A) u_{1}=-\oiiint \frac{\partial p_{1}}{\partial x_{1}} d V+\oiiint \rho f_{1} d V=M
$$

Assuming incompressible, steady flow, Equation 8 reduces to,

$$
M=\rho \oiint(\bar{V} \cdot d A) u_{1}
$$

Applying the integral to the control volume specified in Figure 13 and assuming the control volume is of unit depth, Equation 9 is rewritten as, 


$$
\begin{aligned}
M=-\rho \int_{A}^{B} U_{\infty} & u_{1} d x_{2} \\
& +\rho \int_{B}^{C} u_{2} u_{1} d x_{1}+\rho \int_{C}^{D} U(y) u_{1} d x_{2}-\rho \int_{D}^{A} u_{2} u_{1} d x_{1}
\end{aligned}
$$

Assuming the velocity in the $\mathrm{x}$-direction, $u$, is significantly greater than the velocity in the other axial directions, substituting Equation 11 into Equation 10, and simplifying, the moment of the freestream lost due to the presence of the airfoil, which acts as a turbulent generator, $M$ per unit volume can be written as Equation 32 .

$$
\begin{gathered}
u_{1}=U(y) \\
M=\rho U(y) \int_{C}^{D}\left(U(y)-U_{\infty}\right) d x_{2}
\end{gathered}
$$

The wake is absent of any momentum of its own; therefore, based on the conservation of momentum, the loss in momentum of the freestream is directly related to the boundary layer and separation that occurs due to the viscous interaction between the object in the flow and the freestream. This viscous interaction is what is accountable for the loss of momentum in the freestream, which is simply the drag of the object entrained in the flow. Therefore, the drag on the object, $D$, is equal to the negative value of $M$.

$$
D=-M
$$

Substituting Equation 12 into Equation 13,

$$
D=-\rho U(y) \int_{C}^{D}\left(U(y)-U_{\infty}\right) d x_{2}
$$

The desired quantity is the two-dimensional drag coefficient, which is determined through Equation 14, where $\mathrm{c}$ is the chord.

$$
c_{d}=\frac{D}{\frac{1}{2} \rho U_{\infty}{ }^{2} c}
$$




\subsubsection{Vorticity}

Vorticity allows for the local angular velocity of the flow and the build-up of rotational and vorticial structures to be evaluated. Using the reference frame from Figure 13 [24], the vorticity in the flow was determined through numerical differentiation of the two-dimensional velocity field generated through PIV. The equation for vorticity with respect to a 2-D flow field, assuming steady, incompressible flow is given by Equation 15, where $u$ and $v$ denote the velocity in the $\mathrm{x}$ and $\mathrm{y}$ direction, respectively, and $\omega_{z}$ is the angular velocity of the flow around the z-axis.

$$
\xi_{z}=2 \omega_{z}=\left(\frac{\partial v}{\partial x}-\frac{\partial u}{\partial y}\right)
$$

The partial derivatives of the $u$ and $v$ velocities were evaluated through numerical differentiation utilizing forward, backward, and central differences. The forward and backwards differences were used on the edge velocity vectors of the velocity flow field, for which a data point prior to or subsequent to, respectively, is not present. For all other points, central difference was utilized. Using these differences, the local vorticity was able to be calculated throughout the flow field. The equations for forward difference with respect to $u$ and $v$ are provided in Equations 16 and 17, respectively. The equations for backwards difference with respect to $u$ and $v$ are provided in Equations 18 and 19, respectively. The equations for the central difference with respect to the $u$ and $v$ velocities are provided in Equations 20 and 21, respectively.

$$
\begin{aligned}
& \frac{\partial\left(u_{i, j}\right)}{\partial y}=u_{y}\left(x_{i}, y_{j}\right) \approx \frac{\left(u_{i, j+1}-u_{i, j}\right)}{\Delta y} \\
& \frac{\partial\left(v_{i, j}\right)}{\partial x}=v_{x}\left(x_{i}, y_{j}\right) \approx \frac{\left(v_{i+1, j}-v_{i, j}\right)}{\Delta x} \\
& \frac{\partial\left(u_{i, j}\right)}{\partial y}=u_{y}\left(x_{i}, y_{j}\right) \approx \frac{\left(u_{i, j}-u_{i, j-1}\right)}{\Delta y} \\
& \frac{\partial\left(v_{i, j}\right)}{\partial x}=v_{x}\left(x_{i}, y_{j}\right) \approx \frac{\left(v_{i, j}-v_{i-1, j}\right)}{\Delta x}
\end{aligned}
$$




$$
\begin{aligned}
& \frac{\partial\left(u_{i, j}\right)}{\partial y}=u_{y}\left(x_{i}, y_{j}\right) \approx \frac{\left(u_{i, j+1}-u_{i, j-1}\right)}{2 \Delta y} \\
& \frac{\partial\left(v_{i, j}\right)}{\partial x}=v_{x}\left(x_{i}, y_{j}\right) \approx \frac{\left(v_{i+1, j}-v_{i-1, j}\right)}{2 \Delta x}
\end{aligned}
$$

\subsubsection{Reynolds Shear Stress}

The Reynolds shear stress (sometimes known as just Reynolds stress) is a component of the stress tensor that represents the mean momentum transfer through turbulence. The derivation of the Reynolds stress tensors is discussed below.

Applying Reynolds decomposition, Equation 3, to the conservation of mass, while assuming incompressibility and a Newtonian fluid,

$$
\frac{\partial\left(\overline{U_{i}}+u_{i}^{\prime}\right)}{\partial x_{i}}=0
$$

Taking the temporal average of Equation 22, allows for the derivation of Equation 23, since the time averaged value of the fluctuating velocity is zero.

$$
\frac{\partial \overline{U_{i}}}{\partial x_{i}}=0 \quad \text { and } \quad \frac{\partial u_{i}^{\prime}}{\partial x_{i}}=0
$$

This allows for the continuity equation to be applied to the averaged velocity.

Similarly, Reynolds decomposition can be applied to the conservation of momentum. The conservation of momentum, assuming a Newtonian fluid and an incompressible flow with no external forces, is written as,

$$
\frac{\partial \tilde{u}_{i}}{\partial t}+\tilde{u}_{j} \frac{\partial \tilde{u}_{i}}{\partial x_{j}}=-\frac{1}{\rho} \frac{\partial P_{i}}{\partial x_{i}}+v \frac{\partial^{2} \tilde{u}_{i}}{\partial x_{j}{ }^{2}}
$$

Applying the chain rule, the second term on the left-hand side of Equation 24 can be written as,

$$
\frac{\partial}{\partial x_{j}}\left(\tilde{u}_{i} \tilde{u}_{j}\right)=\tilde{u}_{i} \frac{\partial \tilde{u}_{j}}{\partial x_{i}}+\tilde{u}_{j} \frac{\partial \tilde{u}_{i}}{\partial x_{j}}
$$

Utilizing Equation 25 and applying Reynolds decomposition, the conservation of momentum can be rewritten as, 


$$
\frac{\partial\left(\overline{U_{i}}+u_{i}^{\prime}\right)}{\partial t}+\frac{\partial}{\partial x_{j}}\left(\left(\overline{U_{i}}+u_{i}^{\prime}\right)\left(\overline{U_{j}}+u_{j}^{\prime}\right)\right)=-\frac{1}{\rho} \frac{\partial\left(\bar{P}+p^{\prime}\right)}{\partial x_{i}}+v \frac{\partial^{2}\left(\overline{U_{i}}+u_{i}^{\prime}\right)}{\partial x_{j}{ }^{2}}
$$

The terms on the left-hand side of Equation 13 represent the rate of change of momentum with respect to time and space. The first term on the right-hand side is the momentum imparted on the control volume due to pressure gradients and the second term on the right-hand side is the momentum imparted on the control volume due to shear stress. Temporally averaging Equation 26 and simplifying, knowing the averaged fluctuating component is equal to zero, results in Equation 14.

$$
\frac{\partial \overline{U_{i}}}{\partial t}+\frac{\partial}{\partial x_{j}}\left(\overline{U_{i}} \overline{U_{j}}+\overline{u_{i}^{\prime} u_{j}^{\prime}}\right)=-\frac{1}{\rho} \frac{\partial \bar{P}}{\partial x_{i}}+v \frac{\partial^{2} \overline{U_{i}}}{\partial x_{j}^{2}}
$$

The extra term between Equations 24 and 27, $\overline{u_{i}^{\prime} u_{j}^{\prime}}$, is known as the Reynolds stress tensor. The Reynolds stress tensor describes the velocity fluctuation of the flow velocity by component. The Reynolds stress tensor for a three-dimensional flow is found in Equation 28.

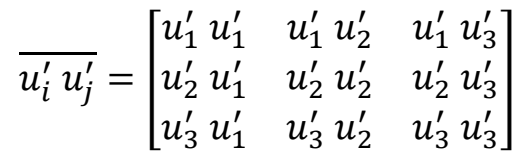

The diagonal terms of the Reynolds stress tensor represent the normal stresses, which contribute minimally to the transport of the mean momentum in most flows. The off-diagonal terms represent the Reynolds shear stress and play the dominant role in the mean momentum transfer by turbulent motion. It is important to note that the mean velocity should remain constant when Reynolds decomposition is used.

\subsection{Proper Orthogonal Decomposition (POD)}

The presence of coherent structures in the flow field were obtained using Proper Orthogonal Decomposition (POD). POD is a form of modal analysis. In aerodynamics, POD is primarily used to extract energetically and dynamically important physical features or modes. Initially introduced for use in fluid mechanics by Lumley [25], POD allows for the coherent 
structures in turbulent flow to be extracted by optimization of the mean square of the velocity in this flow field.

Mathematically, POD starts with Equation 29; where $q(\xi, t)$ is the velocity vector field, $\bar{q}(\xi)$ is the temporal mean of the velocity vector field, $\xi$ is the one, two, or three dimensional discrete spatial points at discrete times, $t$, and $\emptyset_{j}(\xi)$ are the orthogonal modes with $a_{j}$ being their corresponding temporal coefficients [26]. The orthogonal modes are arranged by energy level. Snapshot POD was utilized in this research due to the large spatial size of the data; therefore, the snapshot POD algorithm developed by Sirovich [27] will be discussed.

$$
q(\xi, t)-\bar{q}(\xi)=\sum_{j} a_{j}(t) \emptyset_{j}(\xi)
$$

A series of "snapshots," or vector fields, of the flow field are organized in a series of column vectors, $x(t)$ [26]. The collection of finite-dimension data vectors represents the flow field [26].

$$
x(t)=q(\xi, t)-\bar{q}(\xi) \in \mathbb{R}^{n}, \quad t=t_{1}, t_{2}, \ldots . t_{m}
$$

$x(t)$ is equal to the fluctuating components of the vector field data with the time averaged value subtracted. Using $x(t)$, POD looks to determine the optimal basis vectors that best represent the vector field, i.e. solving for the vectors $\emptyset_{j}(\xi)$ that can represent $q(\xi)$ [26]. This is done by solving for the eigenvectors $\emptyset_{j}$ and eigenvalues $\lambda_{j}$, using Equations 31 and 32, where $R$ is the covariance matrix of vector $x(t)$ [26].

$$
\begin{gathered}
R \emptyset_{j}=\lambda_{j} \emptyset_{j}, \quad \emptyset_{j} \in \mathbb{R}^{n}, \quad \lambda_{1} \geq \lambda_{2} \ldots \geq \lambda_{n} \geq 0 \\
R=\sum_{i=1}^{m} x\left(t_{i}\right) x^{T}\left(t_{i}\right)=X X^{T} \in \mathbb{R}^{n x n}
\end{gathered}
$$

$R$ is typically a $n \times n$, where $n$ was the spatial degrees of freedom, but due to the large spatial size of the data, the eigenvalues are extremely difficult to solve for. Therefore, the spatial modes are recovered from the temporal correlation matrix, as developed by Sirovich [27]. This is done by reducing the snapshots into multiple collection at different discrete time levels into spatial 
size $m \times m$ and solving for the eigenvalues of the smaller sets, $\psi_{j}$, using Equation 33 [27]. The POD modes are then recovered through Equation 34 [27]. Eigenvalue decomposition is only able to be performed on square matrices. Since flow field data typically yields rectangular matrices [26], single value decomposition (SVD) is used instead of eigen value decomposition to determine the eigenvalues and eigenvectors.

$$
\begin{aligned}
& X^{T} X \psi_{j}=\lambda_{j} \psi_{j}, \quad \psi_{j} \in \mathbb{R}^{n}, \quad m \ll n \\
& \emptyset_{j}=X \psi_{j} \frac{1}{\sqrt{\lambda_{j}}} \in \mathbb{R}^{n}, \quad j=1,2, \ldots, m
\end{aligned}
$$

The solved eigenvalues are used to determine the number of modes needed to represent the flow field. This allows the flow field to be represented only by the important modes in terms of the finite and truncated series [26], as mathematically expressed by Equation 35. The temporal coefficients are determined through Equation 36 [26]. By using the modes, the original vector field can be recreated, as shown in Figure 14, from Taira et al. [26]. Even with only using the mean flow and the first few high energy modes, the flow field is well represented.

$$
\begin{gathered}
q(\xi, t)-\bar{q}(\xi) \approx \sum_{j=1}^{r} a_{j}(t) \emptyset_{j}(\xi) \\
a_{j}(t)=\left\{q(\xi, t)-\bar{q}(\xi), \emptyset_{j}(\xi)\right\}=\left\{x(t), \emptyset_{j}\right\}
\end{gathered}
$$

The reconstruction of the instantaneous flow field, shown by Figure 14, is performed through combining the separate modes taking into account the amount of the flow field energy present in each of the modes. A high-dimensional reconstruction can be performed including the high order modes, but a low-dimensional reconstruction, using just the lower order modes is typically able to reconstruct the flow field with great precision. An example of a low-dimensional reconstruction is shown in Figure 14. As shown, only the first three modes are required to reconstruct the instantaneous flow field with high precision. 


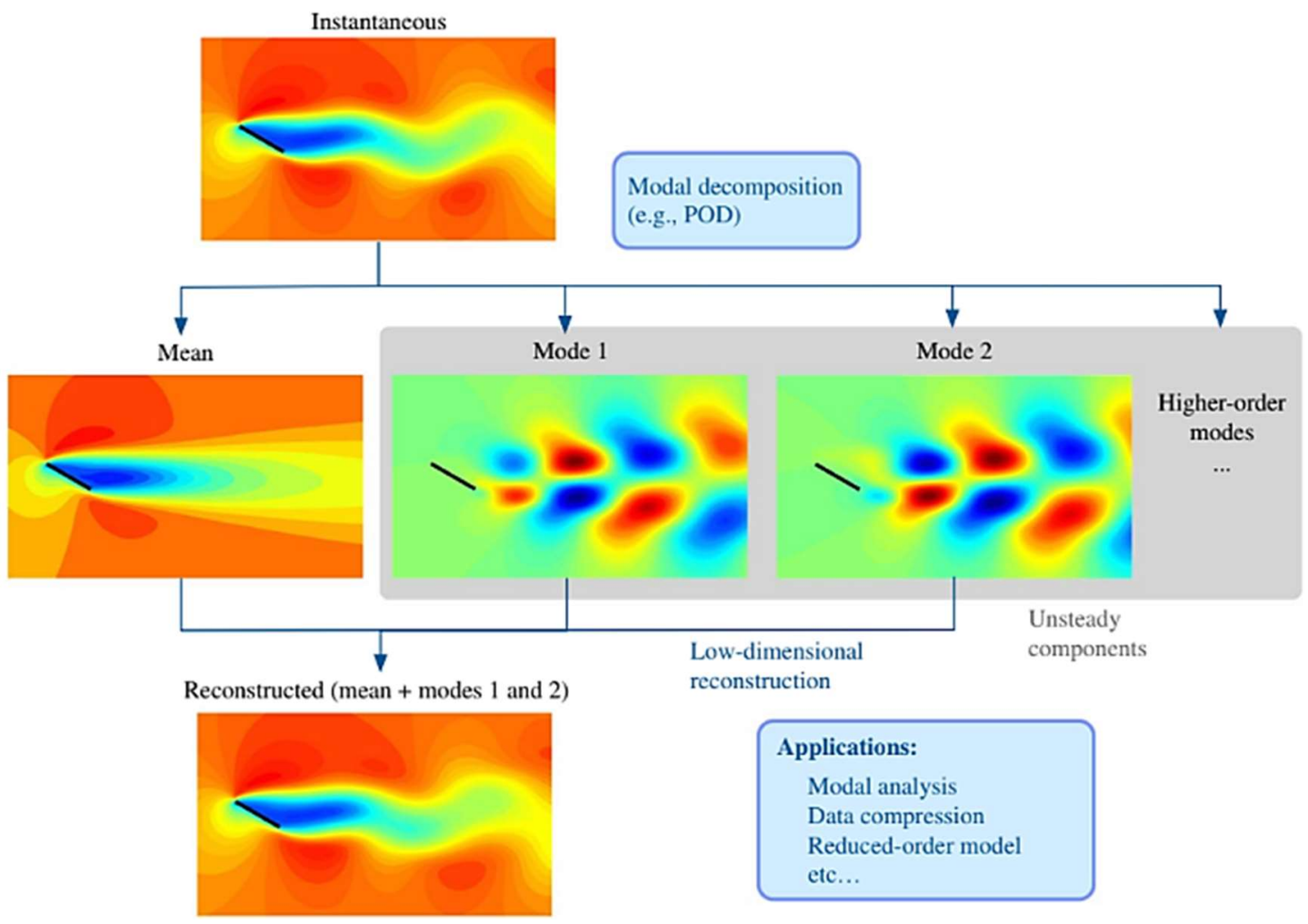

Figure 14: Reconstruction of a 2D instantaneous flow field from Snapshot POD modal analysis [26] 


\section{CHAPTER III}

\section{EXPERIMENTAL SETUP}

All experiments were performed in the University of Dayton - Low Speed Wind Tunnel (UD-LSWT) in the open jet configuration. Force-based experimentation and Particle Image Velocimetry (PIV) were employed to complete the research objectives mentioned in Section 1.6. The specifications of the UD-LSWT, all experimental processes, the iced airfoil models, an overview of PIV, and uncertainty quantification are discussed in this Chapter.

\subsection{University of Dayton Low Speed Wind Tunnel (UD-LSWT)}

The UD-LSWT has a 16:1 contraction ratio, 6 anti-turbulence screens, and can be operated in five different configurations: four different interchangeable test sections $(76.2 \mathrm{~cm} \mathrm{x} 76.2 \mathrm{~cm} \mathrm{x}$ $243.8 \mathrm{~cm}$ ) and an open jet configuration. In all five configurations, the tunnel can obtain a free steam velocity range of $6.7 \mathrm{~m} / \mathrm{s}$ to $40 \mathrm{~m} / \mathrm{s}$ with a turbulence intensity below $0.1 \%$ at $15 \mathrm{~m} / \mathrm{s}$ measured using a hot-wire anemometer. The free stream velocity profile can be varied up to $5 \mathrm{~Hz}$ and over $50 \%$ velocity amplitude using a downstream shuttering system.

\subsection{Horn Ice Geometries and Models}

Three ice accretion geometries: 1) Full scale 2) Scaled down (under SLD conditions) and the corresponding prediction of the ice shape by 3) LEWICE were 3D printed and tested in the UDLSWT for two separate icing conditions. A NACA 0012 airfoil with no ice formation was also tested to act as a baseline. The ice shapes were chosen based on ice shape verification trials from Anderson and Tsao [1], depicted in Figure 15 with the specified icing conditions. These conditions, along with the droplet diameter distribution based on total LWC supplied by the NASA GRC IRT, were used to generate the corresponding LEWICE ice structures. The full-scale ice shape refers to the ice accretion collected on the $183 \mathrm{~cm}$ chord NACA 0012 airfoil and the scaled ice shape refers to the ice accretion collected on the $27 \mathrm{~cm}$ chord NACA 0012 airfoil in this thesis. 
The input files used to generate the LEWICE predicted accretions can be found in Appendix A. The droplet diameter distribution supplied by NASA GRC IRT for an MVD of $170 \mu \mathrm{m}$ can be found in Appendix B. Table 2 summarizes the different ice accretions used in the verification research performed. They are separated into two sets based on their associated stagnation freezing fraction. The geometry of the horn ice accretion varies significantly with $n_{0}$. As such, it was chosen as a parameter of interest in the present investigation to perform aerodynamic verification. It is important to note that the ice shapes are not symmetric. The values in Table 2 were calculated based on a point $0.01 \mathrm{c}$ from the leading edge along the chord. As mentioned, the stagnation freezing fraction has a large influence on the s/c and horn angle of the horn ice shape geometry.

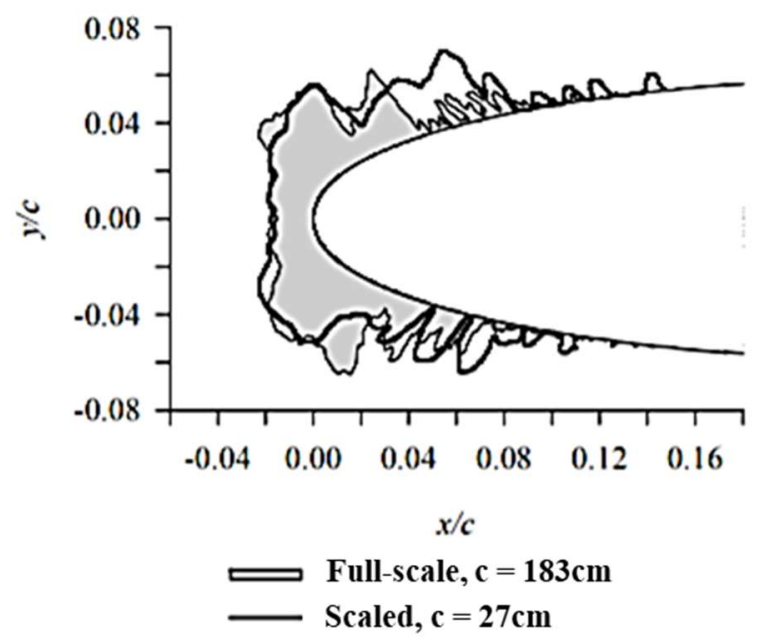

(a) $n_{0}, 0.30$.

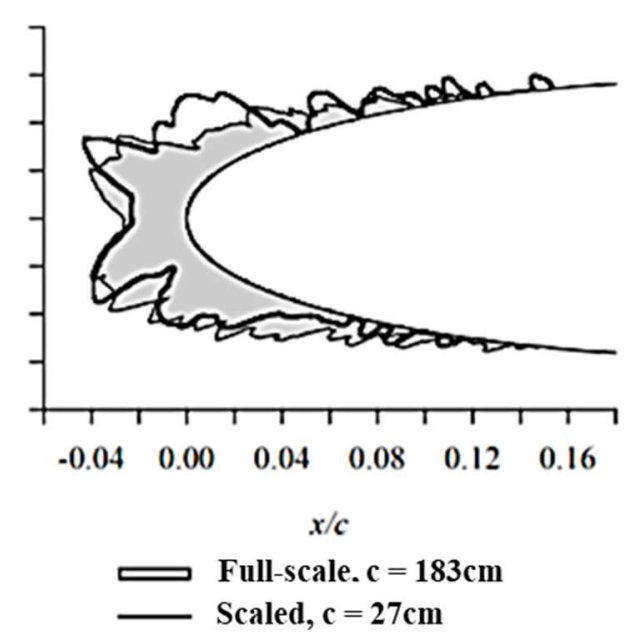

(b) $n_{0}, 0.50$.

\begin{tabular}{|c|c|c|c|c|c|c|c|c|c|c|c|c|c|c|}
\hline & Date/Run & $\begin{array}{c}c, \\
\mathrm{~cm}\end{array}$ & $\begin{array}{l}t_{s l}, \\
{ }^{\circ} \mathrm{C}\end{array}$ & $\begin{array}{l}t_{f o x} \\
{ }^{\circ} \mathrm{C}\end{array}$ & $\begin{array}{l}V, \\
\mathrm{kt}\end{array}$ & $\begin{array}{c}M V D, \\
\mu \mathrm{m}\end{array}$ & $\begin{array}{l}\angle W C, \\
\mathrm{~g} / \mathrm{m}^{3}\end{array}$ & $\underset{\min }{\tau,}$ & $\begin{array}{l}\beta_{0,} \\
\%\end{array}$ & $A_{c}$ & $\beta_{b, A_{c}}$ & $n_{0}$ & $\begin{array}{l}W e_{e_{s}} \\
10^{3}\end{array}$ & $\begin{array}{l}W e_{L}, \\
10^{6}\end{array}$ \\
\hline \multirow[t]{2}{*}{ (a) } & $03-24-05 / 01$ & 182.9 & -12 & -11 & 100 & 170 & 1.45 & 22.9 & 92.3 & 1.93 & 1.78 & 0.30 & 6.90 & 2.35 \\
\hline & $02-28-02 / 01$ & 26.7 & -12 & -2 & 268 & 33 & 0.86 & 2.1 & 92.3 & 1.94 & 1.79 & 0.30 & 9.59 & 2.47 \\
\hline \multirow[t]{2}{*}{ (b) } & $03-24-05 / 03$ & 182.9 & -21 & -20 & 100 & 170 & 1.45 & 22.9 & 92.2 & 1.93 & 1.78 & 0.50 & 6.90 & 2.35 \\
\hline & $02-15-02 / 08$ & 26.7 & -15 & -5 & 276 & 31 & 0.64 & 2.7 & 92.0 & 1.89 & 1.74 & 0.50 & 9.71 & 2.61 \\
\hline
\end{tabular}

Figure 15: Full-scale and scaled non-dimensional ice shapes used for scaling method verification with a) the full-scale and scaled collected ice shapes at $n_{0}=0.3$ and b) the fullscale and scaled collected ice shapes at $n_{0}=0.5[1]$ 
Table 2: Summarization of Ice Shapes used in Verification

\begin{tabular}{|c|c|c|c|c|}
\hline & $\begin{array}{c}\text { Horn } \\
\text { Designation }\end{array}$ & $\Theta,[\mathrm{deg}]$ & $\mathrm{s} / \mathrm{c}$ & $\mathrm{h} / \mathrm{c}$ \\
\hline $\begin{array}{c}\text { Case 1: Baseline } \\
\text { NACA } 0012\end{array}$ & N/A & N/A & N/A & N/A \\
\hline \multicolumn{5}{|c|}{$n_{0}=0.3$} \\
\hline \multirow{2}{*}{ Case 2: Full-scale } & Upper & 80.7 & 0.017 & 0.041 \\
\hline & Lower & 89.4 & 0.020 & 0.047 \\
\hline \multirow{2}{*}{ Case 3: Scaled } & Upper & 79.5 & 0.017 & 0.042 \\
\hline & Lower & 74.1 & 0.015 & 0.039 \\
\hline \multirow{2}{*}{ Case 4: LEWICE } & Upper & 82.3 & 0.018 & 0.029 \\
\hline & Lower & 83.4 & 0.018 & 0.024 \\
\hline \multicolumn{5}{|c|}{$n_{0}=0.5$} \\
\hline \multirow{2}{*}{ Case 5: Full-scale } & Upper & 31.1 & 0.0056 & 0.042 \\
\hline & Lower & 35.1 & 0.0063 & 0.045 \\
\hline \multirow{2}{*}{ Case 6: Scaled } & Upper & 31.8 & 0.0057 & 0.051 \\
\hline & Lower & 35.4 & 0.0064 & 0.048 \\
\hline \multirow{2}{*}{ Case 7: LEWICE } & Upper & 34.9 & 0.0063 & 0.051 \\
\hline & Lower & 39.8 & 0.0073 & 0.043 \\
\hline
\end{tabular}

A model for each cases was designed in SOLIDWorks and 3D printed using Nylon SLS.

The models have a span and chord length of $0.2032 \mathrm{~m}$, providing a model AR of 1 . This chord length was chosen to allow for the preservation of the fine ice features. The span was then calculated based on the maximum allowable forces specified for the ATI Gamma SI-65-5 sensor, see Table 3 , a Reynolds number of 500,000, and a factor of safety of 1.3 . The axial and normal forces were back calculated from the expected lift and drag of a NACA 0012 airfoil at an angle of attack of $15^{\circ}$.

Two-dimensional flow was achieved by adding end plates to the wingtips. The experimental setup will be discussed in the later sections. Overlays of the corresponding ice accretions are graphed in Figures 16 and 17. The ice shapes being compared are referenced by their corresponding stagnation freezing fractions, $n_{0}$. Clear geometric differences are visible. Throughout the paper, data with respect to the full-scale ice accretions, scaled ice accretions, and LEWICE ice accretions will be represented by yellow, blue, and orange, respectively. 


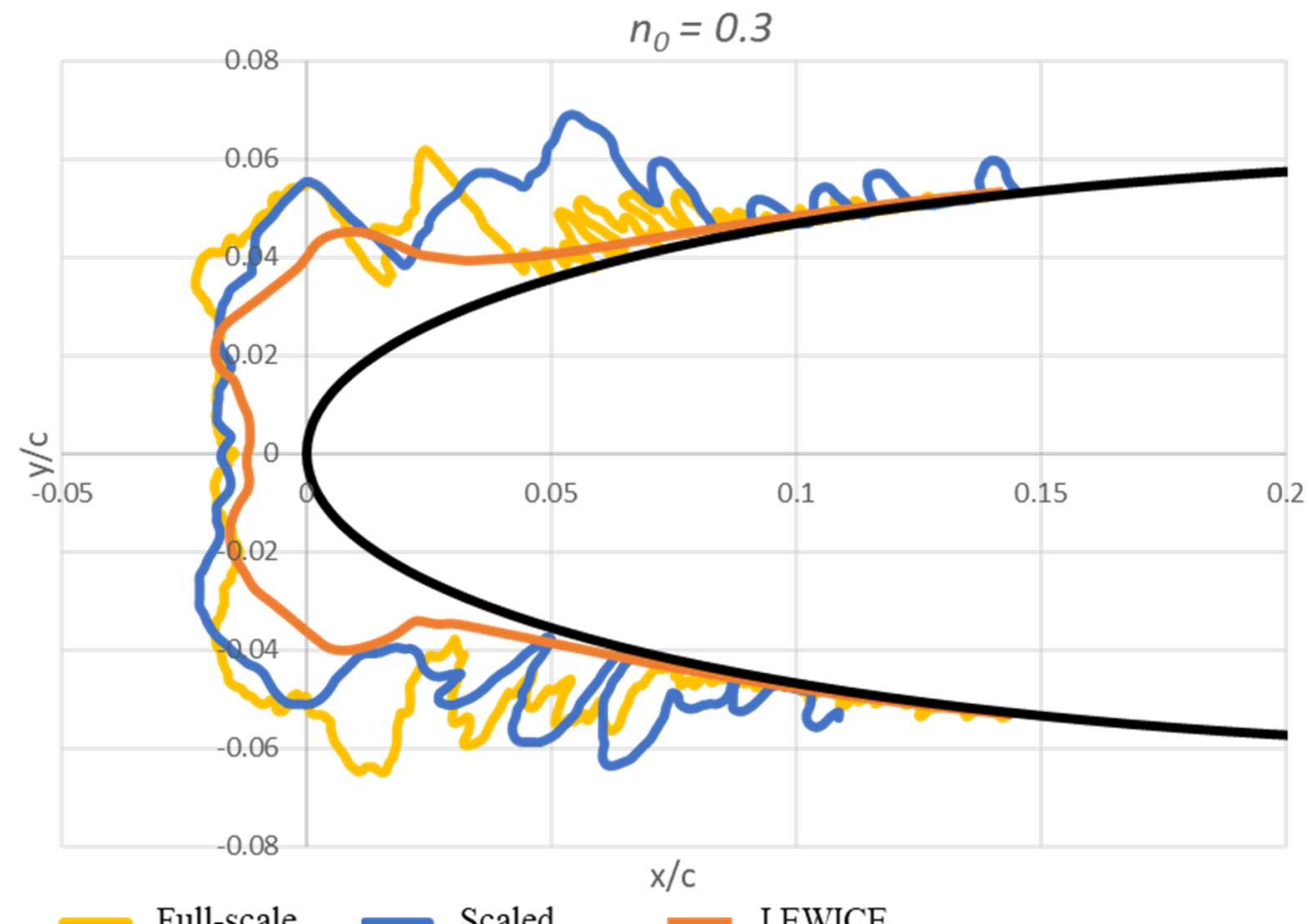

Figure 16: Ice shape overlay of ice accretions collected with $n_{0}=0.3$

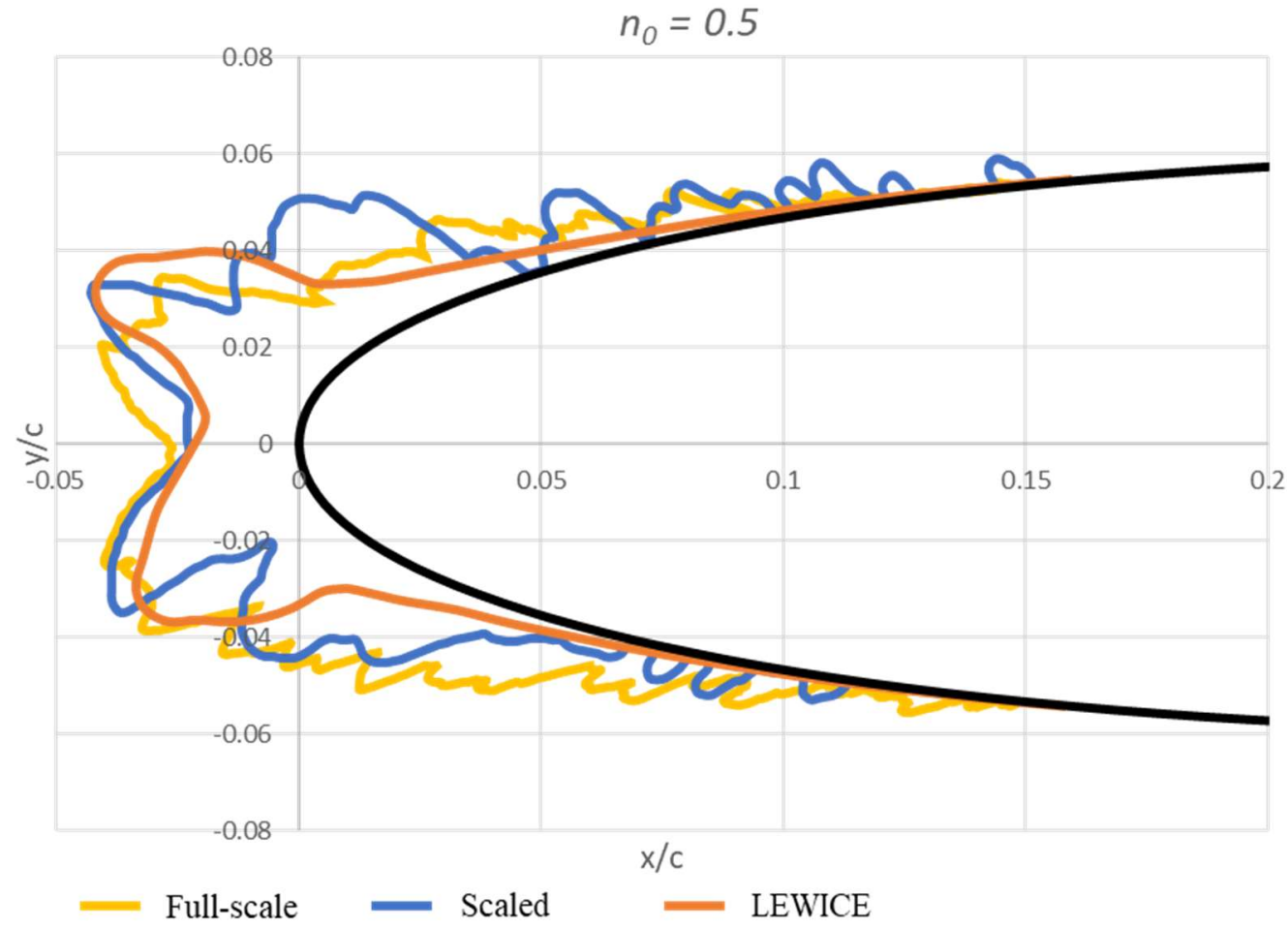

Figure 17: Ice shape overlay of ice accretions collected with $n_{0}=0.5$ 
Figures 18 through 20 depict the models used in testing. Figure 18 provides a top, side, and isometric view of one of the models and Figure 19 shows a picture of all seven models. Nylon SLS was chosen to ensure the fine features of the ice shapes were preserved. A closeup side view of the full-scale $n_{0}=0.3$ ice shape model in Figure 20 shows the intricacy and accuracy of the 3D printed ice shape.
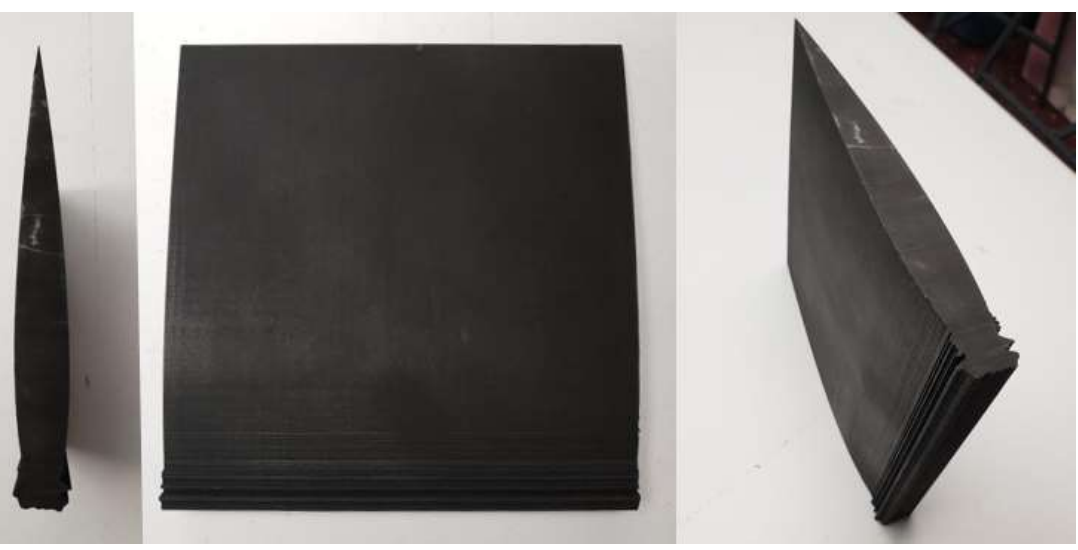

Figure 18: Side, top, and isometric view of $n_{0}=0.3$ full-scale ice shape model

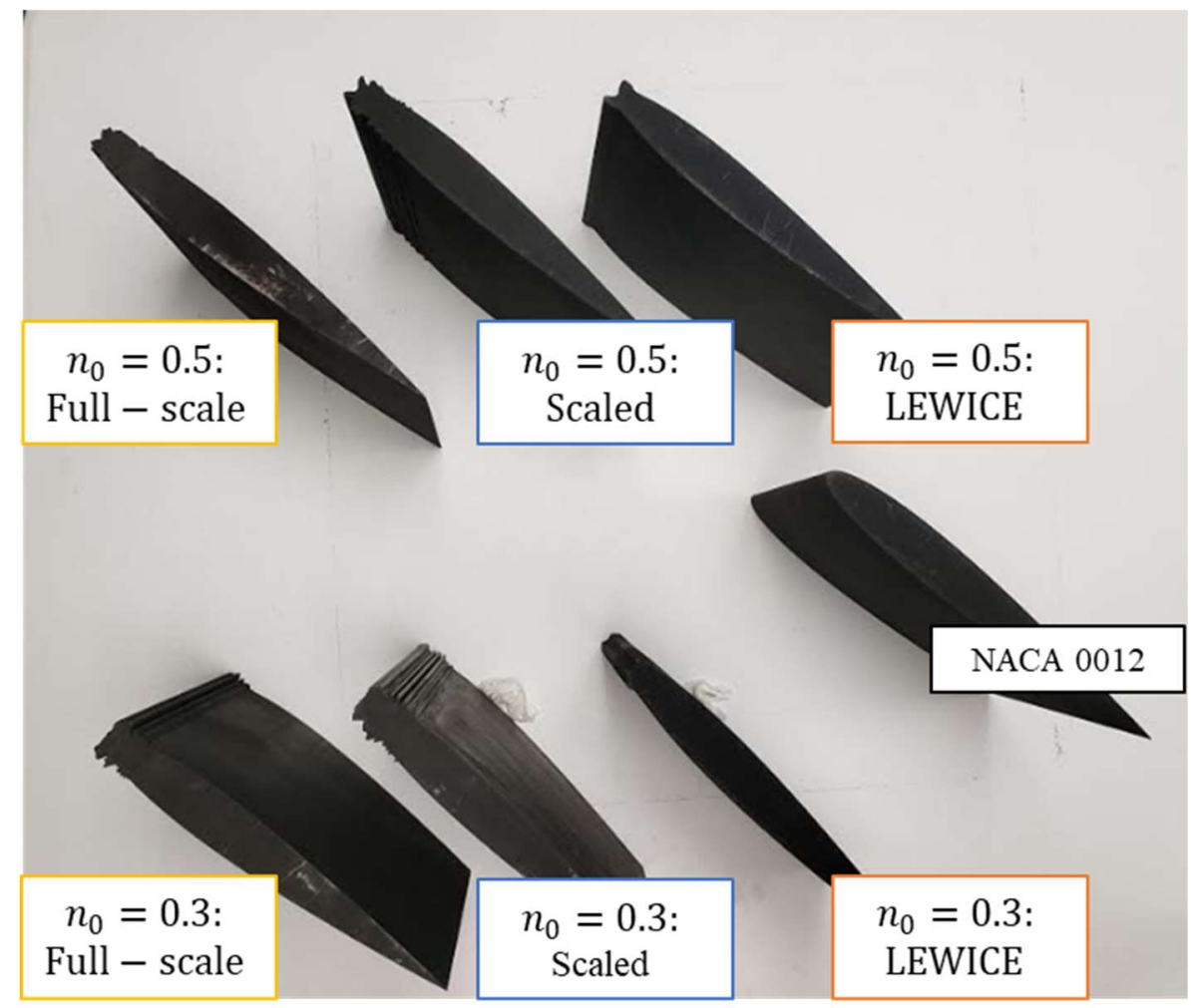

Figure 19: Picture of all seven models used in testing 


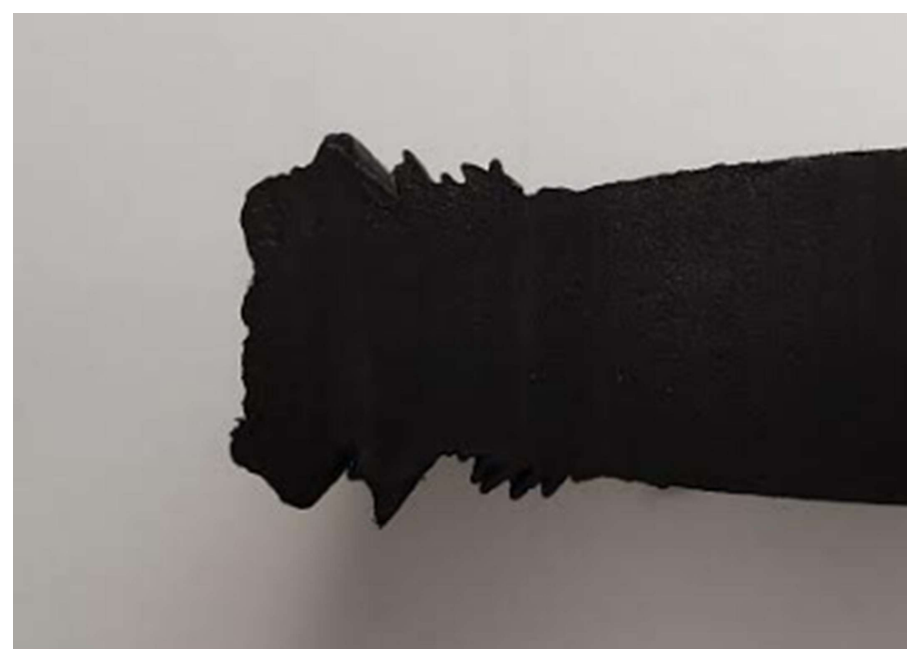

Figure 20: Close up of $n_{0}=0.3$ full-scale ice shape showing preservation of fine ice features during printing

\subsection{Force Based Experimentation}

A schematic of the force-based experimentation is shown in Figure 21 and a picture of actual setup in the UD-LSWT is shown in Figure 22. As shown in the figures, two splitter plates were mounted near the wingtips of the model to generate a two-dimensional flow field and hinder the rollup of the wingtip vortex. However, it is well known that the use of the splitter plates at the wingtips doesn't always guarantee an ideal two-dimensional flow in the enclosed model. Three dimensional effects will always be present due to the splitter plate being entrained in the flow, resulting in an increased aspect ratio (AR) effect rather than an ideal two-dimensional flow. In order to accommodate for the three-dimensional effects, the slope of the linear portion of the lift curves were compared to the Helmbold low aspect ratio straight-wing equation, Equation 37 [28], where $A R$ is the wing aspect ratio, $C_{L_{\alpha}}$ is the 3 -dimensional lift curve slope, and $C_{l_{\alpha}}$ is the theoretical twodimensional lift curve slope of $2 \pi$.

$$
C_{L_{\alpha}}=C_{l_{\alpha}} \frac{A R}{\left(\frac{C_{l_{\alpha}}}{\pi}\right)+\sqrt{\left(\frac{C_{l_{\alpha}}}{\pi}\right)^{2}+A R^{2}}}
$$




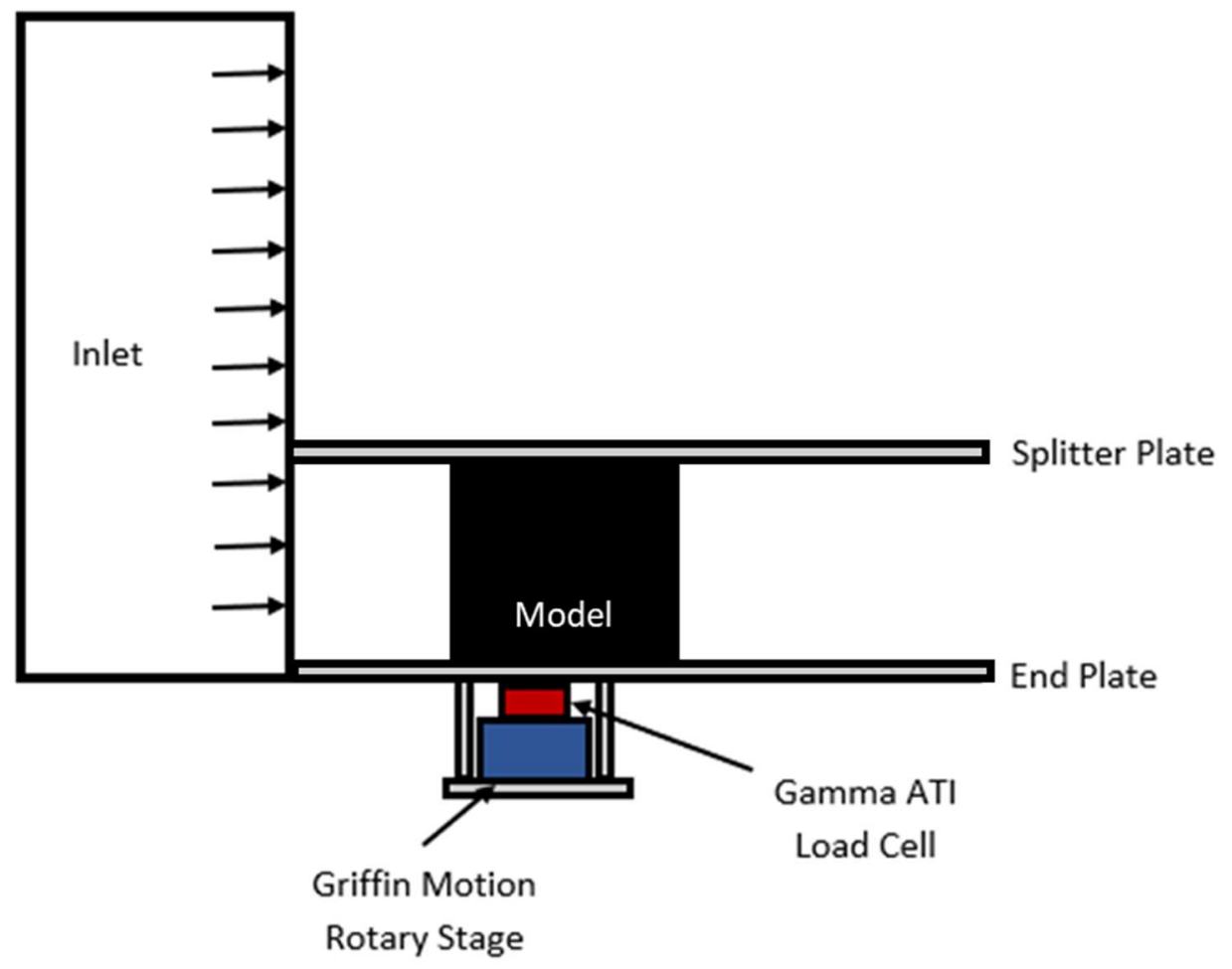

Figure 21: Force-based experimentation in the UD-LSWT schematic

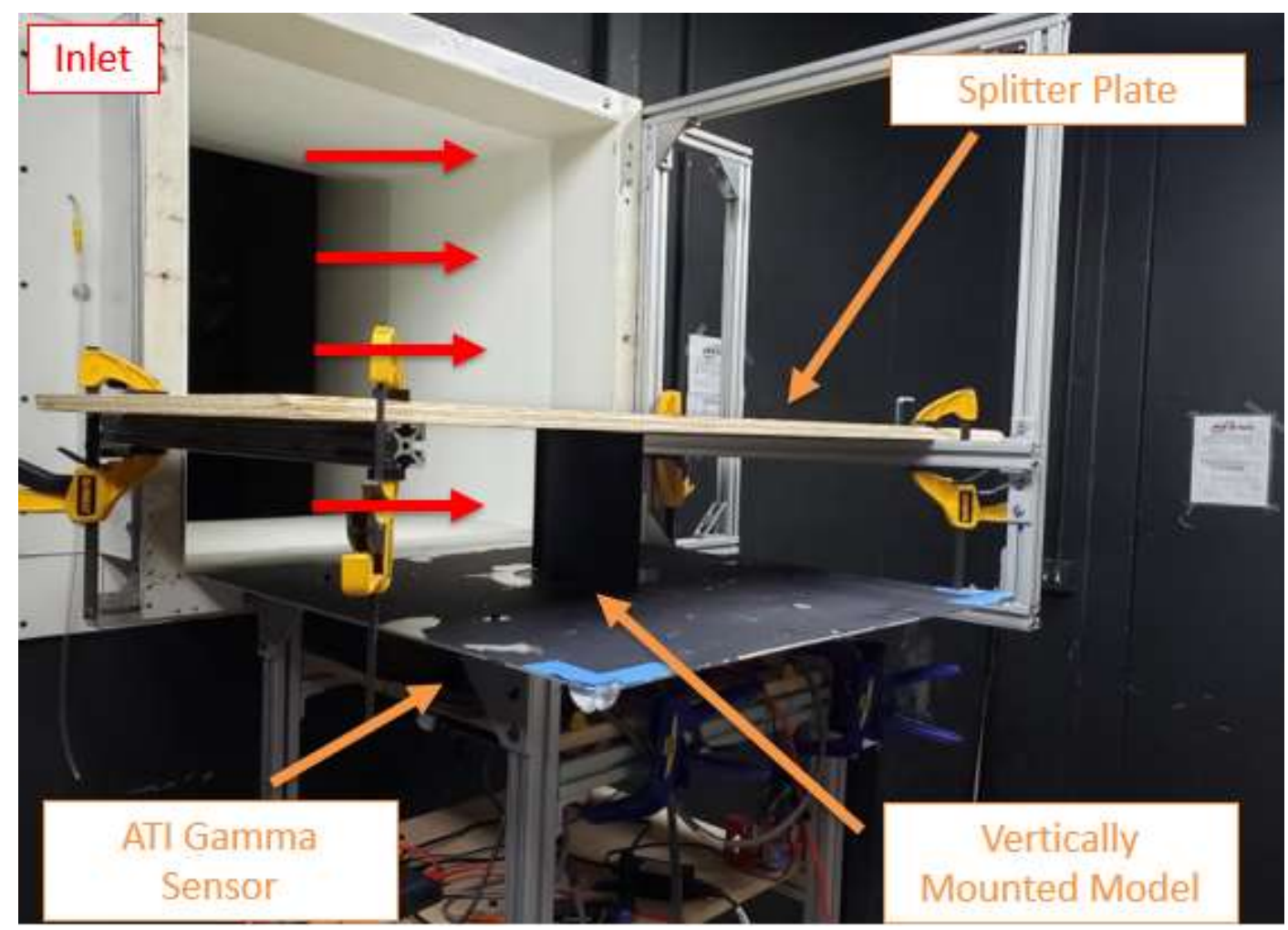

Figure 22: Force-Based Experimentation in the UD-LSWT 
Force based experimentation was performed for all nine cases across a Reynolds number range from 300,000 to 500,000 to determine the Reynolds number sensitivity of the aerodynamic coefficients of wings with ice accretions. The Reynolds number insensitivity of the aerodynamics coefficients of the wings with ice accretions were reported in [8][13][14], in the Reynolds number range of $2 \mathrm{e} 6$ to $10 \mathrm{e} 6$. However, the insensitivity needs to be verified in the comparatively low Reynolds number testing conditions in the current investigation.

Axial and normal force data for each case at the different Reynolds numbers were collected using a six component ATI Gamma SI-65-5 sensor across a $-15^{\circ}$ to $15^{\circ}$ AOA sweep. From the collected axial and normal force data, lift and drag coefficients were calculated and plotted as a function of angle of attack. This allowed for the quantification of the aerodynamic penalties associated with each ice shape. Each trial was repeated for both increasing and decreasing angle of attack, in order to check for hysteresis and to ensure repeatability. The test matrix of the forcebased experiments is shown in Table 3.

Table 3: Force Based Experimentation Test Matrix

\begin{tabular}{|c|c|c|c|}
\hline $\begin{array}{c}\text { Reynolds } \\
\text { Number }\end{array}$ & Cases & AOA Sweep & $\begin{array}{c}\text { Number of } \\
\text { Trials }\end{array}$ \\
\cline { 1 - 1 } 300,000 & \multirow{2}{*}{ All Cases } & $\begin{array}{c}-15^{\circ} \text { to } 15^{\circ} \\
\left(1^{\circ} \text { increments }\right)\end{array}$ & 3 \\
\cline { 1 - 2 } 400,000 & 300,000 & \\
\hline
\end{tabular}

The specifications for the ATI Gamma SI-65-5 Sensor are provided in Table 4 [29]. The normal and axial force was measured using the $\mathrm{X}$ and $\mathrm{Y}$ axis of the sensor which have similar magnitude ranges. The resolution of the forces for both $\mathrm{X}$ and $\mathrm{Y}$ axes are the same.

Table 4: Specifications of ATI Gamma SI-65-5 Sensor [29]

\begin{tabular}{|c|c|c|c|c|c|c|}
\hline & Fx (N) & Fy (N) & Fz (N) & Tx (Nm) & Ty (Nm) & Tz (Nm) \\
\hline Range & 65 & 65 & 200 & 5 & 5 & 5 \\
\hline Resolution & $1 / 80$ & $1 / 80$ & $1 / 40$ & $1 / 13333$ & $1 / 13333$ & $1 / 13333$ \\
\hline
\end{tabular}


Data acquisition was performed by obtaining data at multiple sampling rates to ensure insensitivity of the force data to sampling frequency. The results presented were captured at a sampling rate of $100 \mathrm{~Hz}$.

\subsection{Particle Image Velocimetry (PIV)}

\subsubsection{Overview}

Particle Image Velocimetry (PIV) is an experimental technique used to estimate velocity in a flow field by seeding the flow with tracer particles, illuminating them utilizing a concentrated light source, and capturing their motion over a defined time interval with a high-speed camera. A schematic of the PIV process is shown in Figure 23 [30].

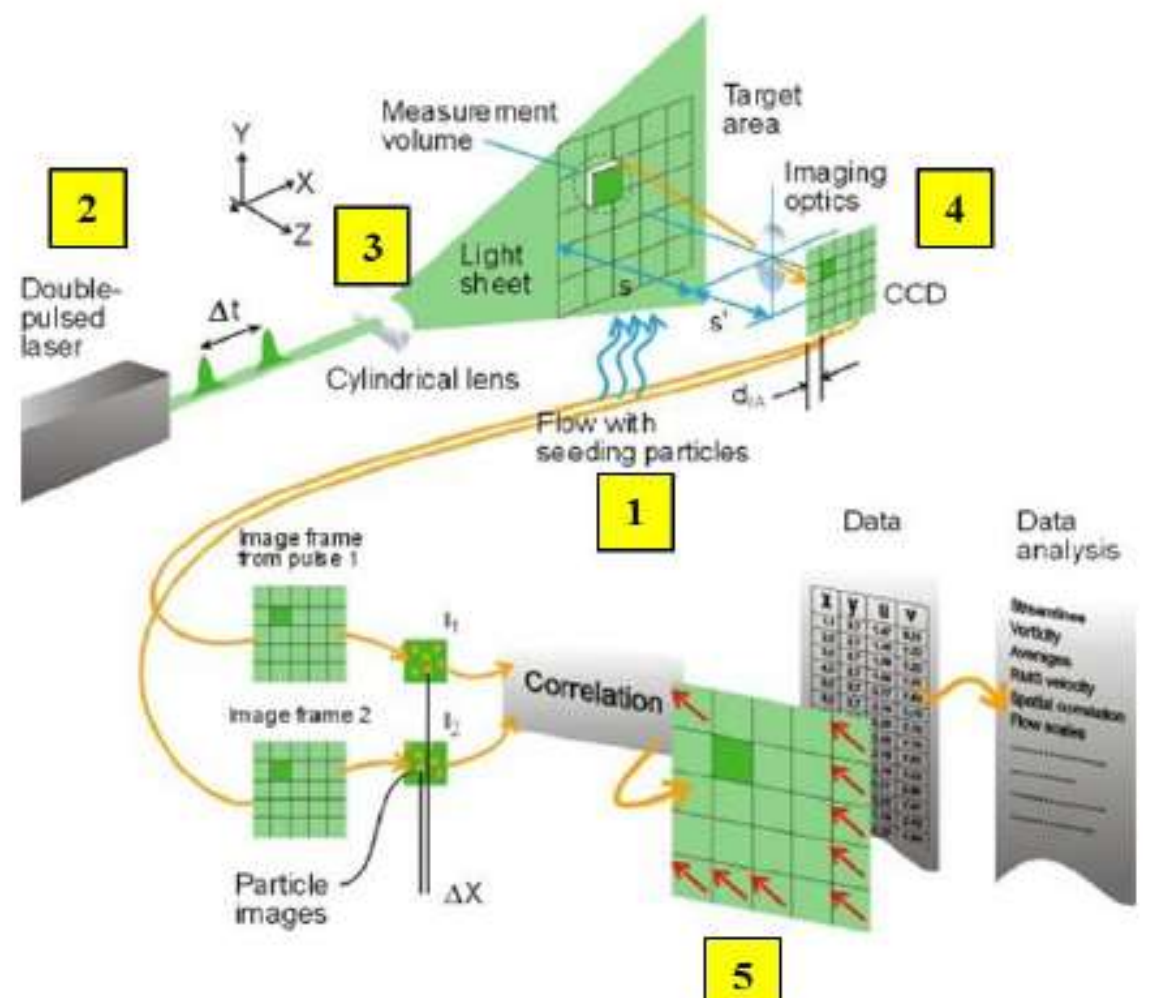

Figure 23: Basic schematic for PIV [30]

An inherent assumption in PIV is that the tracer particles follow the flow religiously. How well a particle follows the flow is quantified using the Stokes number, which is the ratio of the particle's momentum response time to the flow field time scale. The Stokes number is calculated 
using Equation 38, where $\tau$ is the relaxation time, $\rho$ is the density of the air, $d_{d}$ is the particle diameter, $d_{c}$ is the characteristic diameter of the model, $U_{\infty}$ is the freestream velocity, and $\mu$ is the dynamic viscosity. A Stokes number significantly less than 1 is desired to ensure the particle's motion closely follows the flow.

$$
S t k=\frac{\rho d_{d}^{2} U_{\infty}}{18 \mu d_{c}}
$$

The seeding in the UD-LSWT was performed using a Vicount smoke seeder with glycerinbased oil, resulting in tracer particles with a mean diameter of 0.2 microns. The Stokes number of the glycerin-based oil was calculated based on the experimental parameters. Sea level conditions were assumed for the density and dynamic viscosity, $1.225 \mathrm{~kg} / \mathrm{m}^{3}$ and $1.81 \mathrm{e}-5 \mathrm{~kg} /(\mathrm{m} \bullet \mathrm{s})$, respectfully. The freestream velocity of all PIV experiments was $22.06 \mathrm{~m} / \mathrm{s}$, based on a Reynolds number of 300,000 . The thickness of the models, based on the NACA 0012 profile used, was .0245 $\mathrm{m}$. This resulted in a Stokes number of $1.35 \mathrm{e}-7$, which is significantly less than 1 , as desired.

The laser used in PIV is typically of class IVb or greater to illuminate the seed particles. The seeding particles were illuminated by a planar laser sheet, as shown in Figure 23, produced by directing the UD-LSWT $200 \mathrm{~mJ} /$ pulse Nd: YAG frequency doubled laser (Quantel Twins CFR 300) through a plano-convex lens.

The illuminated particles produce Mie scattering, which is captured with a high-speed camera. This requires the laser and camera to be triggered simultaneously. This was done in the UD-LSWT using a Quantum composer pulse generator. In each test case, 1500 image pairs were obtained for the free-shear PIV and 1000 image pairs were obtained and processed for the on-body PIV to satisfy the statistical requirements for properly resolving turbulent flow characteristics.

The PIV images were processed using Innovative Scientific Solutions Incorporated's (ISSI) Digital Particle Image Velocimetry (DPIV) software. DPIV determines the local velocities by dividing the gathered image pairs into interrogation regions, as shown in the schematic in Figure 23 , and then applying a cross-correlation algorithm to determine the movement of the particles in 
the interrogation regions. A generic formula for cross-correlation provided by Raffel et al. [31] is given in Equation 39.

$$
R[r, s]=\frac{1}{N^{2}} \sum_{i=1}^{N} \sum_{j=1}^{N}\left(I^{\prime}[i, j]-\bar{I}\right)\left(I^{\prime \prime}[i+r, j+s]-\bar{I}\right)
$$

$N$ is the number of pixels in the interrogation region, $I^{\prime}$ is the intensity of the particles in the first image and $I$ ' 'is the intensity of the particles in the second image of the image pair, $\bar{I}$ is the mean intensity of the image, which is subtracted to reduce the background noise, $i$ and $j$ are the length and width of the interrogation region, respectively, and $r$ and $s$ are imitation variables [23]. Using this algorithm, the image pairs are cross-correlated. If the particles between the first image and the second image coincide, the correlation of the interrogation region is strong. This is represented by a strong signal peak, as illustrated in Figure 24.

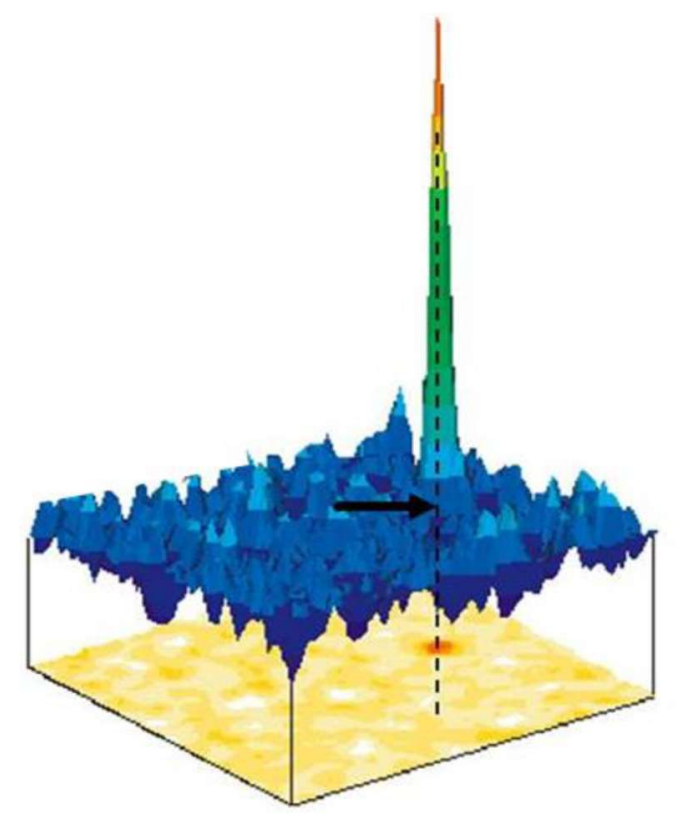

Figure 24: Illustration of a PIV image pair correlation peak [31]

A total of two iterations were performed during PIV processing with 64x64-pixel interrogation regions in the first iteration and 32x32-pixel interrogation regions in the second iteration with an interrogation window overlap of 50\%. A median filter of extremely low strength, allowing for twelve standard deviations to be included, was applied to filter out any incorrect 
vectors. This allowed for greater than $99.9 \%$ of the data to remain. To ensure the median filter did not alter the flow field, the RMS values with and without the filter were compared.

\subsubsection{On-body PIV Setup in the UD-LSWT}

On-body PIV was completed to study the flow field near and around the ice shape allowing for assessment of the ice shape effect on the local aerodynamics. This allowed for studying the separation bubble size, the buildup of vorticity due to the shear layer created by the separation bubble, flow separation, reattachment, and shedding. It also allowed for the assessment of secondary separation points, if present, due to the large feather region ice accretions commonly found in high MVD conditions. The test matrix for the on-body PIV is presented in Table 5. Figure 25 shows a schematic of the on-body setup in the UD-LSWT. In order to simulate two-dimensional flow, as best as possible, an optics glass plane was used as the splitter plate between the camera and model. The glass splitter was able to be mounted directly against the end surface of the model.

Table 5: On-body PIV Test Matrix

\begin{tabular}{|c|c|c|c|c|c|}
\hline \multicolumn{2}{|c|}{ On-Body } & \\
\hline $\begin{array}{c}\text { Reynolds } \\
\text { Number }\end{array}$ & $\begin{array}{c}\text { Freestream } \\
\text { Velocity, [m/s] }\end{array}$ & Cases & $\begin{array}{c}\text { AOA } \\
\text { Sweep }\end{array}$ & $\begin{array}{c}\text { Number } \\
\text { of } \\
\text { Iterations }\end{array}$ & $\begin{array}{c}\text { Image } \\
\text { Pairs } \\
\text { Captured }\end{array}$ \\
\hline 300,000 & 22.06 & All Cases & $\begin{array}{c}\left(3^{\circ}\right. \\
\text { increments })\end{array}$ & 2 & 1000 \\
\hline
\end{tabular}




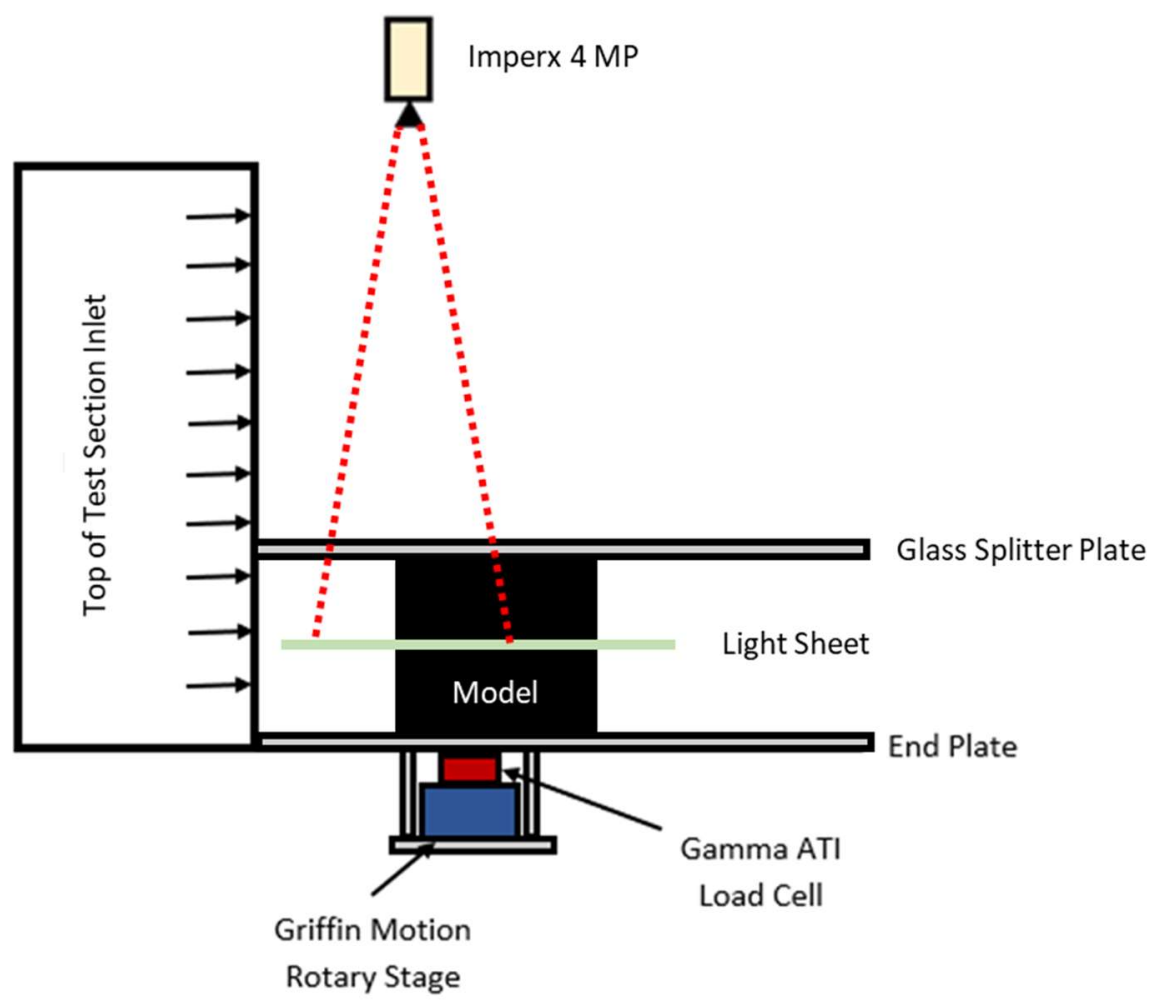

Figure 25: Schematic of on-body PIV setup in UD-LSWT

The on-body PIV parameters are presented in Table 6. The magnification factor was calculated using Equation 40, where $\mathrm{f}$ is the $105 \mathrm{~mm}$ lens focal length, and $\lambda$ is the distance of the lens from the camera sheet. The $\Delta t$ was optimized based on achieving a 10 to 11 particle pixel displacement in the freestream flow, while maintaining a minimum of 4 to 5 particle pixel displacement in the separation bubble. The position of the FOV allowed for the separation bubble to be studied, while still allowing for complete capture of flow separation at stall. Following Table 6 is a schematic showing the FOV with respect to the model in Figure 26.

The actual setup of the on-body PIV is pictured in Figure 27. The laser sheet opened from beneath the model. Due to the placement of the laser sheet, image collection could only be performed on one surface of the model at a time. To collect PIV images on the other surface, the whole setup was flipped around the centerline of the tunnel, while keeping the PIV parameters the same. The processed upper and lower image pairs were then stitched together to study the complete 
near ice accretion flow field. The images were stitched by finding a region of overlapping velocity vectors, see Figure 26, between the corresponding processed upper and lower surface PIV data, using MATLAB. The streamwise velocity, vorticity, and Reynolds stress contours were then overlapped and stitched based on the positioning determined through evaluating the velocity vectors.

$$
\psi=\frac{\lambda}{f}
$$

Table 6: On-body PIV Parameters

\begin{tabular}{|c|c|c|c|c|c|c|c|}
\hline \multicolumn{1}{|c|}{$\begin{array}{c}\text { Imperx } 4 \\
\text { MP } \\
\text { CCD } \\
\text { dim., } \\
\text { [pixel } \mathrm{x} \\
\text { pixel }]\end{array}$} & $\begin{array}{c}\text { FOV } \\
{[\mathrm{mm} x} \\
\mathrm{mm}]\end{array}$ & $\begin{array}{c}\text { FOV in } \\
\text { terms of } \\
\text { chord }\end{array}$ & $\begin{array}{c}\text { Spatial } \\
\text { Resolution } \\
\text { of FOV, } \\
\text { [pixel/m] }\end{array}$ & $\Delta \mathrm{t},[\mu \mathrm{s}]$ & $\lambda,[\mathrm{m}]$ & $f,[\mathrm{~m}]$ & $\psi$ \\
\hline $\begin{array}{c}2048 \mathrm{x} \\
2048\end{array}$ & $\begin{array}{c}169 \mathrm{x} \\
169\end{array}$ & $\begin{array}{c}.832 \mathrm{c} x \\
.832 \mathrm{c}\end{array}$ & 12118 & 36 & 1.32 & 0.1 & 13.2 \\
\hline
\end{tabular}

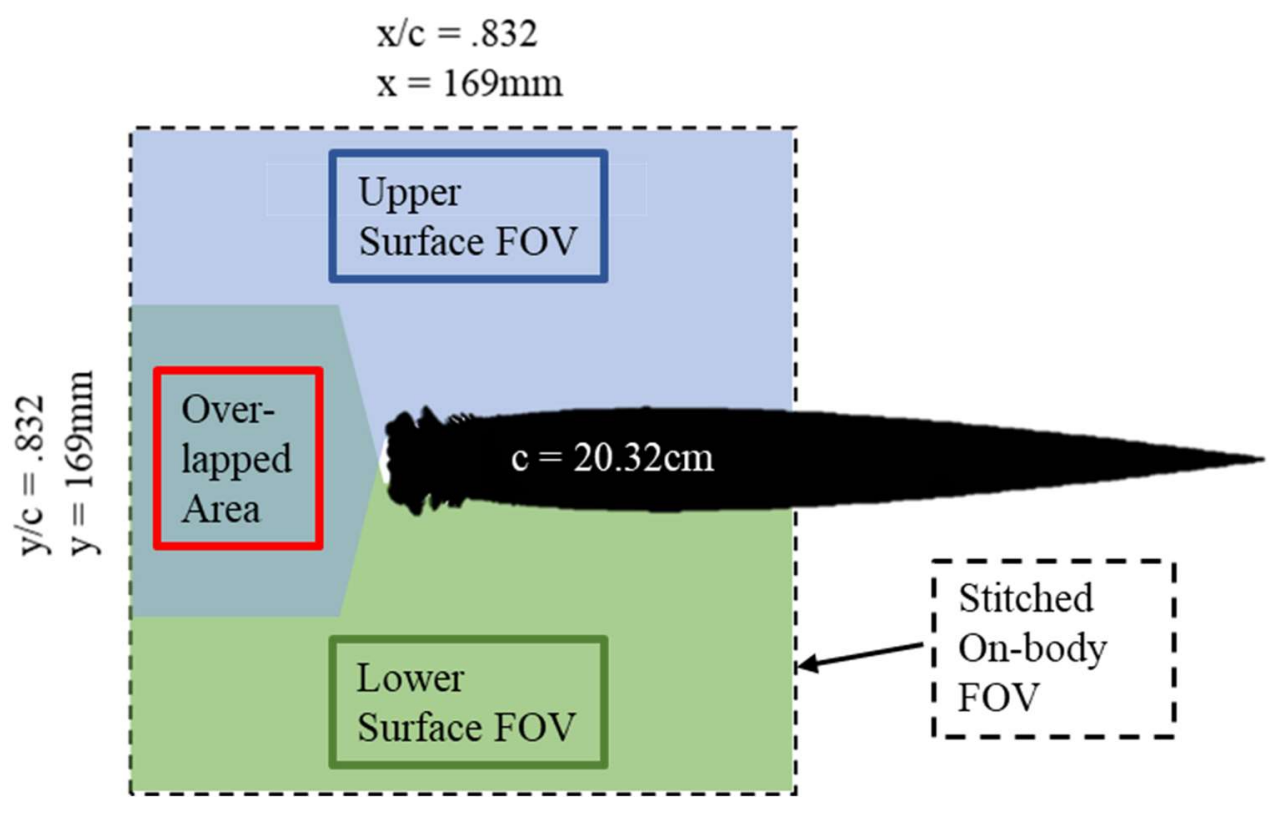

Figure 26: On-body FOV position with respect to the model schematic. The upper and lower surface PIV was performed separately. 


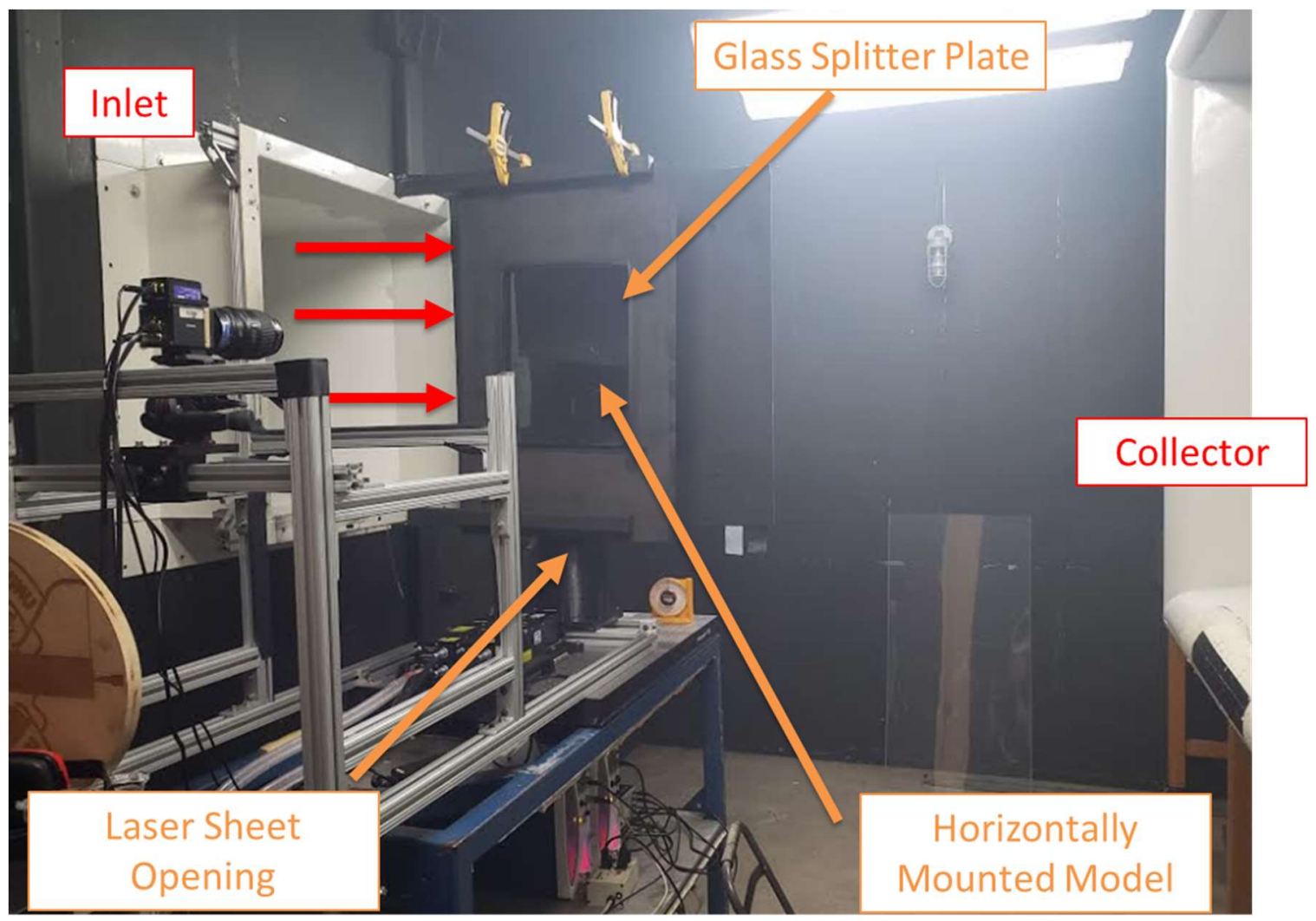

Figure 27: On-body PIV setup in UD-LSWT

\subsubsection{Free Shear Layer PIV Setup in UD-LSWT}

The near wake free shear layer was studied to assess the effect of ice accretions on the airfoil's flow field. The near wake flow characteristics are strongly coupled with the dynamics of the upper and lower surface boundary layers and can be used to determine if the scale and LEWICE accretions faithfully simulate the corresponding upper and lower surface boundary layer characteristics of the full-scale ice accretion. Studying the near wake location allowed for the size of the wake to be assessed, along with the vorticity, the Reynolds stress, and the coherent structures present in the wake. Determining the mean streamwise velocity in the wake allowed for the drag of the iced airfoil to be determined using the momentum deficit principle.

The test matrix for the free shear layer PIV is presented in Table 7. A schematic of the onbody setup in the UD-LSWT is shown in Figure 28. In order to simulate two-dimensional flow, as best as possible, a thin acrylic sheet with a refraction index similar to optical glass was used as the 
splitter plate between the camera and model. The acrylic splitter was able to be mounted directly against the end surface of the model.

Table 7: Free Shear Layer PIV Test Matrix

\begin{tabular}{|c|c|c|c|c|c|}
\hline \multicolumn{7}{|c|}{ Near Wake Free Shear Layer } \\
\hline $\begin{array}{c}\text { Reynolds } \\
\text { Number }\end{array}$ & $\begin{array}{c}\text { Freestream } \\
\text { Velocity, }[\mathrm{m} / \mathrm{s}]\end{array}$ & Cases & $\begin{array}{c}\text { AOA } \\
\text { Sweep }\end{array}$ & $\begin{array}{c}\text { Number } \\
\text { of } \\
\text { Iterations }\end{array}$ & $\begin{array}{c}\text { Image } \\
\text { Pairs } \\
\text { Captured }\end{array}$ \\
\hline 300,000 & 22.06 & All Cases & $\begin{array}{c}-14^{\circ} \text { to } 14^{\circ} \\
\left(2^{\circ}\right. \\
\text { increments })\end{array}$ & 2 & 1500 \\
\hline
\end{tabular}

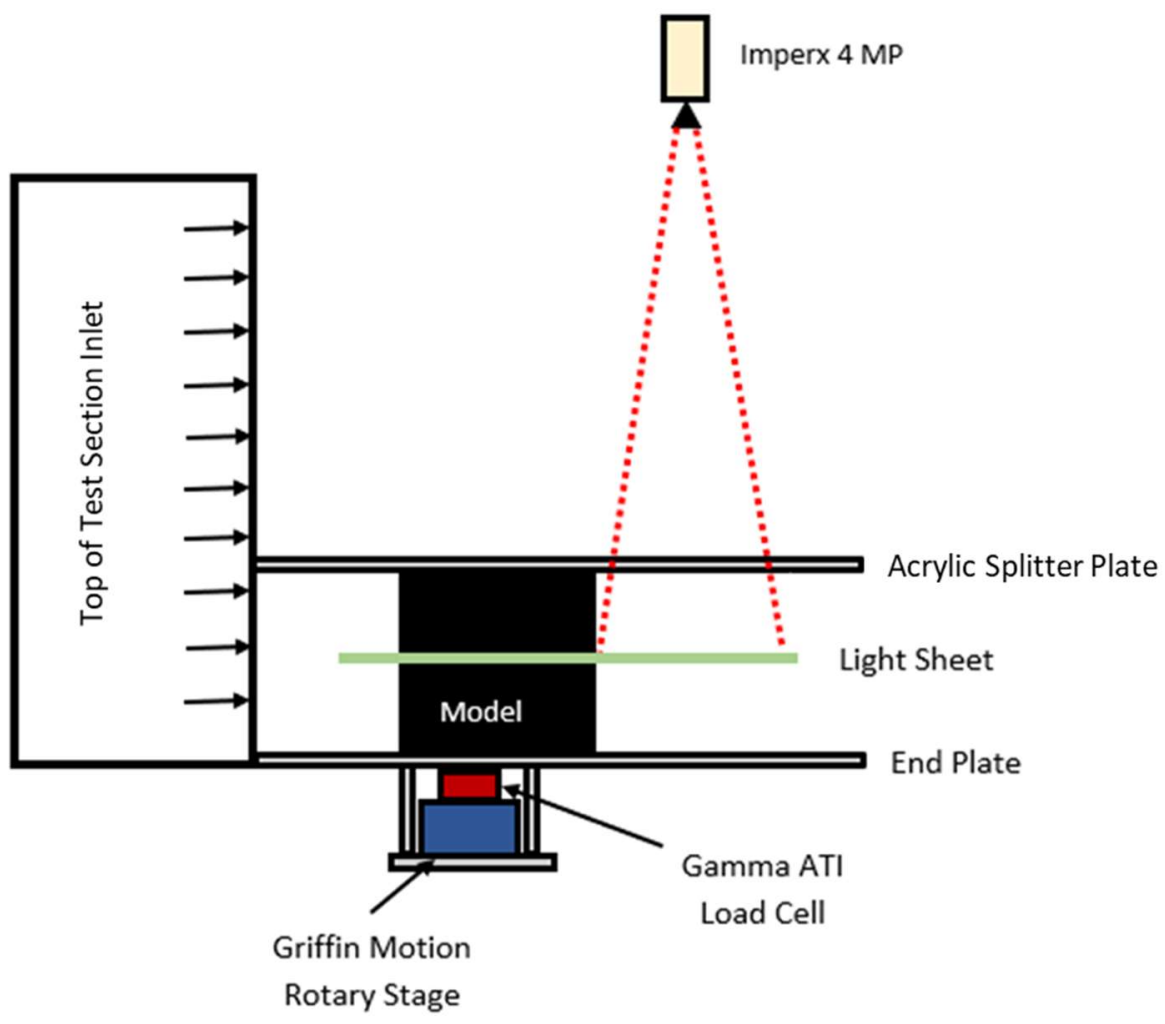

Figure 28: Schematic of free shear layer setup in UD-LSWT

The free shear PIV parameters are presented in Table 8. The magnification factor was calculated using Equation 36. The same $105 \mathrm{~mm}$ lens was used in collecting the free shear layer PIV images as for the on-body PIV image capture. The $\Delta t$ was optimized based on achieving a 10 to 11 particle pixel displacement in the freestream flow, while maintaining a minimum of 4 to 5 
particle pixel turbulent region of the wake. The position and size of the FOV allowed for the wake to be completely captured with the freestream velocity still in view. Following Table 8 is a schematic showing the FOV with respect to the model in Figure 29.

Table 8: Free Shear Layer PIV Parameters

\begin{tabular}{|c|c|c|c|c|c|c|c|}
\hline \multicolumn{8}{|c|}{ Near Wake Free Shear Layer } \\
\hline $\begin{array}{c}\text { Imperx } \\
4 \mathrm{MP} \\
\text { CCD } \\
\text { dim., } \\
\text { [pixel x } \\
\text { pixel] }\end{array}$ & $\begin{array}{c}\text { FOV } \\
{[\mathrm{mm} \mathrm{x}} \\
\mathrm{mm}]\end{array}$ & $\begin{array}{c}\text { FOV in } \\
\text { terms of } \\
\text { chord }\end{array}$ & $\begin{array}{c}\text { Spatial } \\
\text { Resolution } \\
\text { of FOV, } \\
\text { [pixels } / \mathrm{m} \text { ] }\end{array}$ & $\Delta \mathrm{t},[\mu \mathrm{s}]$ & $\lambda,[\mathrm{m}]$ & $f,[\mathrm{~m}]$ & $\psi$ \\
\hline $\begin{array}{c}2048 \mathrm{x} \\
2048\end{array}$ & $\begin{array}{c}155 \mathrm{x} \\
155\end{array}$ & $\begin{array}{c}.758 \mathrm{c} \\
\times .758 \mathrm{c}\end{array}$ & 13213 & 40 & 1.21 & 0.1 & 12.1 \\
\hline
\end{tabular}

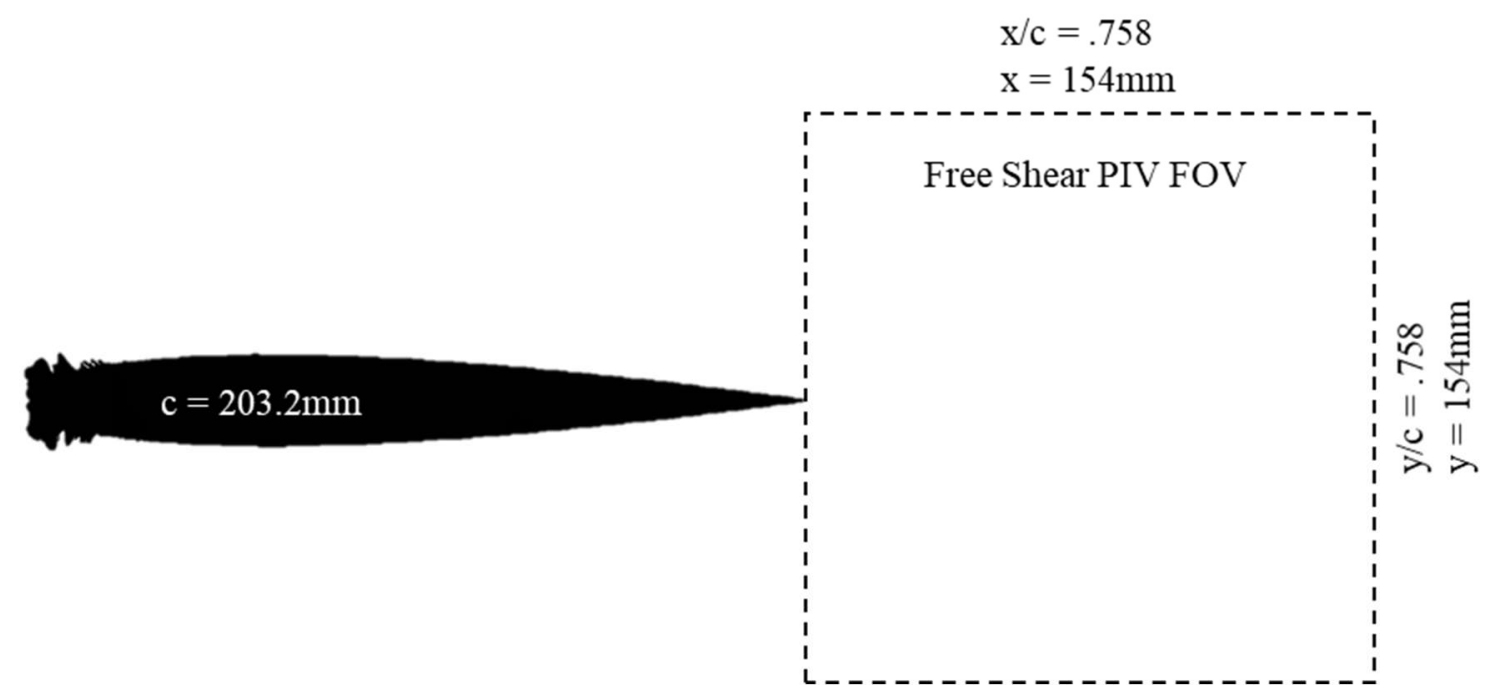

Figure 29: Free Shear Layer FOV position with respect to the model schematic

The actual free shear layer PIV setup in the UD-LSWT is pictured in Figure 30. The laser sheet opened from above the model. Due to this, reflections off the floor had to be mitigated. Anti- 
reflective gaffer tape was positioned in line with the laser sheet on the floor to mitigate the reflections.

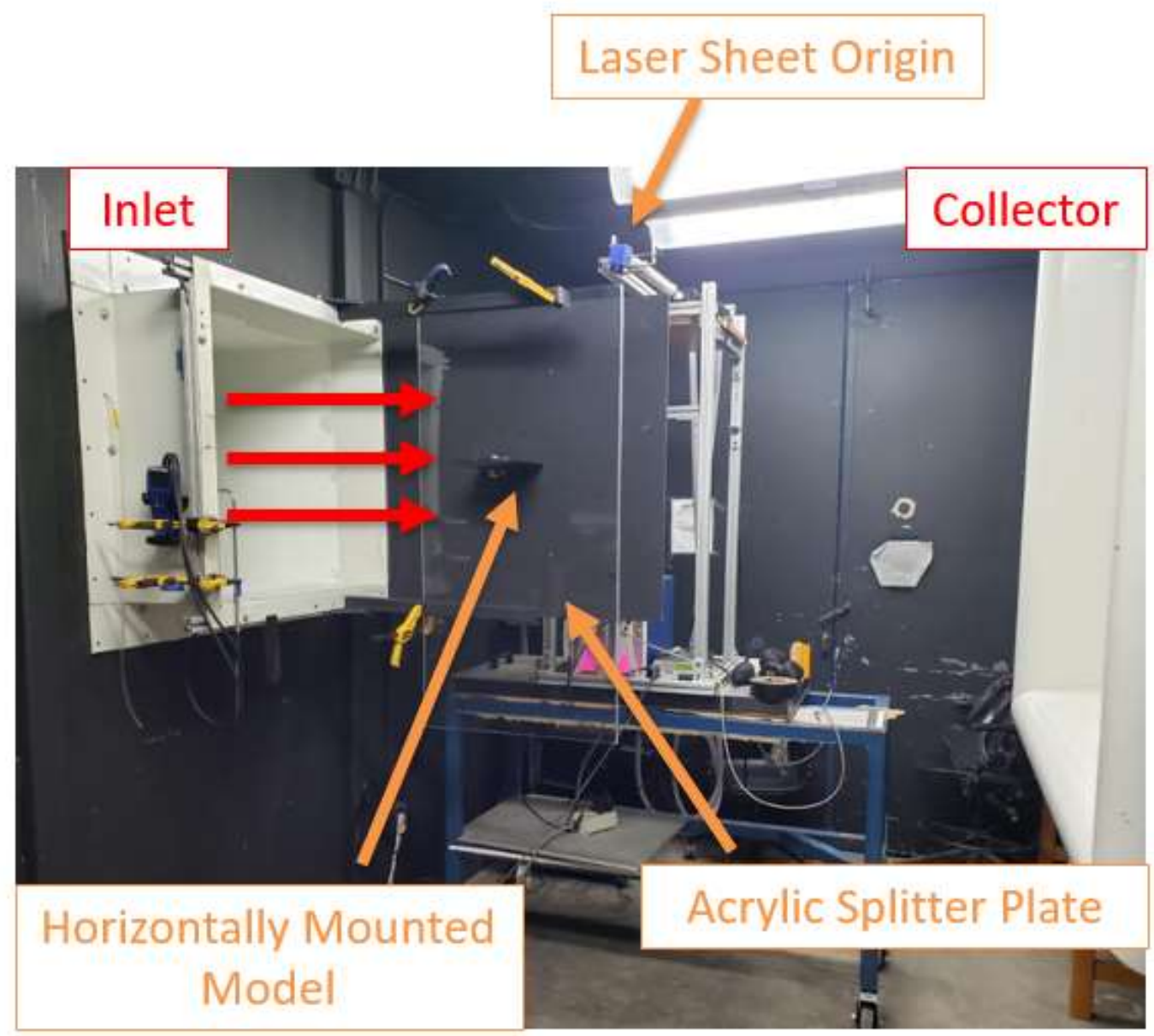

Figure 30: Free Shear Layer PIV setup in UD-LSWT

\subsection{Uncertainty Analysis}

\subsubsection{Force-Based Experimentation}

The uncertainty quantified in this section is due to the instrumentation used in the forcebased experimentation. Bias error was minimized through biasing the ATI Gamma SI-65-5 force transducer prior to data collection. Random error was minimized through temporal averaging of collected data at each angle of attack and testing for the repeatability of the data collected. The procedure was held constant for each model with minimal variation of the setup. Though this does 
not necessarily mitigate the error, it does ensure the procedural error is held constant throughout the trials. Calibration error was minimized, as all equipment was calibrated by the manufacturer.

Quantifying the uncertainty associated with the instrumentation includes evaluating the uncertainty of the ATI Gamma SI-65-5 force transducer, the Griffin Motion Rotary Stage, and the TSI/Alnor 5825 Micromanometer. The Kline-McClintock equation for determining total uncertainty, Equation 41, was used to evaluate the uncertainty associated with the aerodynamic coefficients determined from force-based experimentation. $W_{y}$ is the total instrumental uncertainty, $\frac{d f}{d x_{i}}$ is the derivative of a given equation with respect to a variable for which instrumentation provides a value for, $w_{i}$ is the provided uncertainty of that measured variable by instrumentation error, and the subscript $i$ refers to the measured variable being evaluated. The Kline-McClintock uncertainty analysis assumes the measurements are independent, the probability function of $x_{i}$ is Gaussian, and the confidence level of the measurements are the same.

$$
W_{y}=\sqrt{\left(\frac{d f}{d x_{1}} w_{1}\right)^{2}+\left(\frac{d f}{d x_{2}} w_{2}\right)^{2}+\left(\frac{d f}{d x_{3}} w_{3}\right)^{2}+\cdots}
$$

The normal and axial forces measured were used to evaluate the coefficients of lift and drag at different angles of attack. In order to calculate the coefficients, lift and drag forces were first determined using Equations 42 and 43, respectively. Therefore, the uncertainty in these forces was needed to determine the uncertainties of the respective coefficients. Uncertainty in the lift and drag forces is associated with the ATI Gamma SI-65-5 force transducer and the Griffin Motion Rotary Stage. The values for the uncertainty of the axial force, $A$, and the normal force, $N$, are based on the ATI Gamma SI-65-5 force transducer specifications provided in Table 4 [29]. The uncertainty of the angle of attack, controlled by the Griffin Motion rotary stage, is $1 / 66^{\circ}$ [32].

$$
\begin{aligned}
& L=N \cos (\alpha)-A \sin (\alpha) \\
& D=N \sin (\alpha)+A \cos (\alpha)
\end{aligned}
$$


The total uncertainties of the coefficients were calculated based on Equations 44 and 45 . This allows for the uncertainty with respect to the dynamic pressure measured by the TSI/Alnor 5825 Micromanometer and the 3D printing of the models to be determined. The uncertainty in the measured dynamic pressure is specified as $\pm 1 \%$ of the measured value [33] and the uncertainty in the printing was conservatively assumed to be $0.5 \mathrm{~mm}$. The total uncertainty was evaluated with the Kline-McClintock Analysis and is provided for the coefficients of lift and drag in the form of error bars under the force-based results.

$$
\begin{aligned}
& C_{L}=\frac{L}{q S} \\
& C_{D}=\frac{D}{q S}
\end{aligned}
$$

\subsubsection{Particle Image Velocimetry}

The estimated mean uncertainty in the flow field associated with the PIV experimentation was determined using the method specified by Lazar et al. [34]. The method specified evaluates the total PIV uncertainty, Equation 46, by individually evaluating the four primary sources of error; equipment (instrumentation), particle dynamics, sampling, and image analysis (processing).

$$
\varepsilon_{\text {tot }}=\sqrt{\varepsilon_{\text {Equip }}^{2}+\varepsilon_{P D}^{2}+\varepsilon_{\text {Sampling }}^{2}+\varepsilon_{\text {Proc }}^{2}}
$$

The uncertainty of the equipment was evaluated using Equation 47 [34], where $w_{u}$ is the total uncertainty due to the equipment used. The variable descriptions for Equation 47, along with the measured and uncertainty values from experimentation are provided in Table 9 . The uncertainty of the calibration scale image plane length, $L 1$, and the image distortion due to aberrations, $L 2$, were specified as 1 pixel and $0.5 \%$ of the total length of the calibration scale, respectively, by Lazar et al. [34]. The image distortion due to aberrations, L2, was conservatively estimated by Lazar et al. based on comparing scaling magnification factors formed with different calibration scale lengths. Lazar et al. went on to state that the estimation based on $0.5 \%$ of the total length of the calibration scale is a conservative assumption applicable to standard PIV setups [34]. As such, the 
value of $0.5 \%$ is used in the uncertainty analysis for the PIV test setup. $\tilde{u}$ was specified as the average experimental freestream velocity, $22.06 \mathrm{~m} / \mathrm{s}$. The uncertainty due to the instrumentation was calculated to be $0.64 \%$

Table 9: PIV Equipment Uncertainty Parameters

\begin{tabular}{|c|c|c|c|c|c|c|}
\hline Category & Parameter & Description & $\begin{array}{c}\text { Value, } \\
y_{i}\end{array}$ & Units & $\begin{array}{c}\text { Uncertainty, } \\
\mathrm{w}_{\mathrm{i}}\end{array}$ & Units \\
\hline \multirow{4}{*}{ Calibration } & $l$ & $\begin{array}{l}\text { Calibration scale } \\
\text { physical length }\end{array}$ & 169 & $\mathrm{~mm}$ & 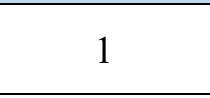 & $\mathrm{mm}$ \\
\hline & $\mathrm{L} 1$ & $\begin{array}{l}\text { Calibration scale image } \\
\text { plane length }\end{array}$ & 2048 & pixel & 1 & pixel \\
\hline & $\mathrm{L} 2$ & $\begin{array}{l}\text { Image distortion due to } \\
\text { aberrations }\end{array}$ & 2048 & pixel & 10.24 & pixel \\
\hline & $\lambda$ & $\begin{array}{c}\text { Distance from } \\
\text { calibration scale to lens }\end{array}$ & 1.17 & $\mathrm{~m}$ & 5 & $\mathrm{~mm}$ \\
\hline \multirow{2}{*}{ Timing } & $\mathrm{t} 1$ & Laser pulse timing & 36 & um & 1.5 & ns \\
\hline & $\mathrm{t} 2$ & $\begin{array}{l}\text { Accuracy of delay } \\
\text { generator }\end{array}$ & 36 & um & 1.5 & ns \\
\hline
\end{tabular}

$$
=\sqrt{w_{u}\left[\left(\frac{1}{L 1} w_{l}\right)^{2}+\left(-\frac{l}{L 1} w_{L 1}\right)^{2}+\left(-\frac{l}{L 2} w_{L 2}\right)^{2}+\left(\frac{1}{\lambda L 1} w_{\lambda}\right)^{2}\right]+\left(-\frac{\tilde{u}}{\Delta t} \frac{l}{L 1}\right)^{2}\left[w_{t 1}{ }^{2}+w_{t 1}{ }^{2}\right]}
$$

Due to the significantly less than one Stokes number calculated in the PIV overview section, the uncertainty associated with particle dynamics is considered negligible compared to the other sources. Therefore, the next uncertainty to be evaluated was associated with sampling. Using the root mean squared (RMS) value of deviation is a common way to estimate the uncertainty in a measurement. Lazar et al. [34] points out that PIV processing software, such as DPIV, computes the velocity RMS of deviation from the mean velocity for every interrogation grid location. Essentially, this is done by calculating a mean velocity at every interrogation window by averaging the results of the complete set of image pairs and then computing the RMS of the individual image pair velocity deviations from the mean for a specified interrogation grid location. By dividing the RMS of deviation value by the mean and multiplying by one-hundred, an estimated percent uncertainty based on the RMS of deviation is calculated at every interrogation grid location. 
The maximum percent uncertainty in the flow field of the full-scale ice accretion collected at $n_{0}=0.3$ at an AOA of $-12^{\circ}$ was then determined. This PIV case was used to the complexity of the flow field, comparatively to the other cases, and the on-body PIV having a lower signal to noise ratio than the free shear layer PIV. This resulted in an estimated maximum sampling uncertainty of $0.97 \%$.

The last uncertainty to be evaluated was associated with the image processing performed through DPIV. Since the PIV technical suggestions and experimental guidelines specified in Lazar et al. [34], where all followed during experimentation and process, the DPIV processing of the images showed strong cross-correlation of the image pairs, with average signal to noise ratio (SNR) for the on-body being greater than three for all cased and angles of attack. Lazar et al. [34] found the uncertainty due to processing using DPIV to be less than $1 \%$ for the majority of vectors for a significantly more complex flow than that being analyzed in this investigation. As was performed during the second iteration of the DPIV processing performed in this investigation, Lazar et al. [34] determined the uncertainty due to processing using DPIV using a 32x32-pixel interrogation region with an interrogation window overlap of $50 \%$. Since the same software with similar specified processing parameters was used for processing the PIV images in the current investigation, the mean uncertainty due to processing was conservatively assumed to be $1 \%$. This results in an overall mean uncertainty of $1.53 \%$ in the flow field velocities determined using PIV, approximately $+/-$ $0.338 \mathrm{~m} / \mathrm{s}$ in the freestream flow.

Based on the evaluation performed, uncertainty due to processing and sampling were determined to be the largest sources, both equal to $\sim+/-1 \%$ of flow field velocities. The uncertainty due to the equipment used in the collection of the PIV images was only $+/-0.64 \%$. Since the uncertainty due to particle dynamics was negligible based on the significantly less than one Stokes number, the dominant sources of uncertainty were the sampling and processing. 


\section{CHAPTER IV}

\section{FORCE BASED EXPERIMENTATION AND MOMENTUM DEFICIT RESULTS}

The effect of the different ice accretions on the aerodynamic coefficients (lift and drag) are discussed in this chapter. The chapter includes coefficient of lift and drag results from the forcebased experimentation of the seven different airfoil cases. The full-scale, scaled, and LEWICE ice accretions collected at $n_{0}=0.3$ and $n_{0}=0.5$ conditions are compared separately. The momentum deficit results obtained from PIV measurements of the free shear layer was also used to determine the two-dimensional drag coefficient to corroborate the drag coefficient results obtained from force-based testing. Throughout the section, whenever the ice shapes are directly compared, the full-scale, scaled, and LEWICE ice shapes are represented by yellow, blue, and orange, respectively. With respect to annotations in figures, the annotation arrow color is blue if the comparison is being made between the scaled and full-scale ice shapes and the arrow color is orange if the comparison is being made between the LEWICE and full-scale ice shapes. If the comparison is being made between the full-scale and both the scaled and LEWICE ice shapes, the arrow is yellow.

\subsection{Effect of Reynolds Number}

Force based experimentation was performed at three separate Reynolds numbers ranging from 300,000 to 500,000 , as specified in Table 3, to quantify the effect of Reynolds number on the aerodynamic coefficients associated with each ice shape. The lift coefficient curves for each of the ice shapes at the varying Reynolds numbers are provided in Figure 31. The coefficient curves show the coefficients are of the same magnitude and trends across the Reynolds number range, except at high angles of attack post $12^{\circ}$. To evaluate the effect of the Reynolds number on the change in lift compared to the baseline NACA 0012, Equation 50 was used. Figure 32 includes the normalized lift coefficient devotion for each of the ice accretion cases. 


$$
\text { Norm. } C_{l}=\frac{C_{l, i c e d}-C_{l, N A C A}}{C_{l, N A C A}}
$$
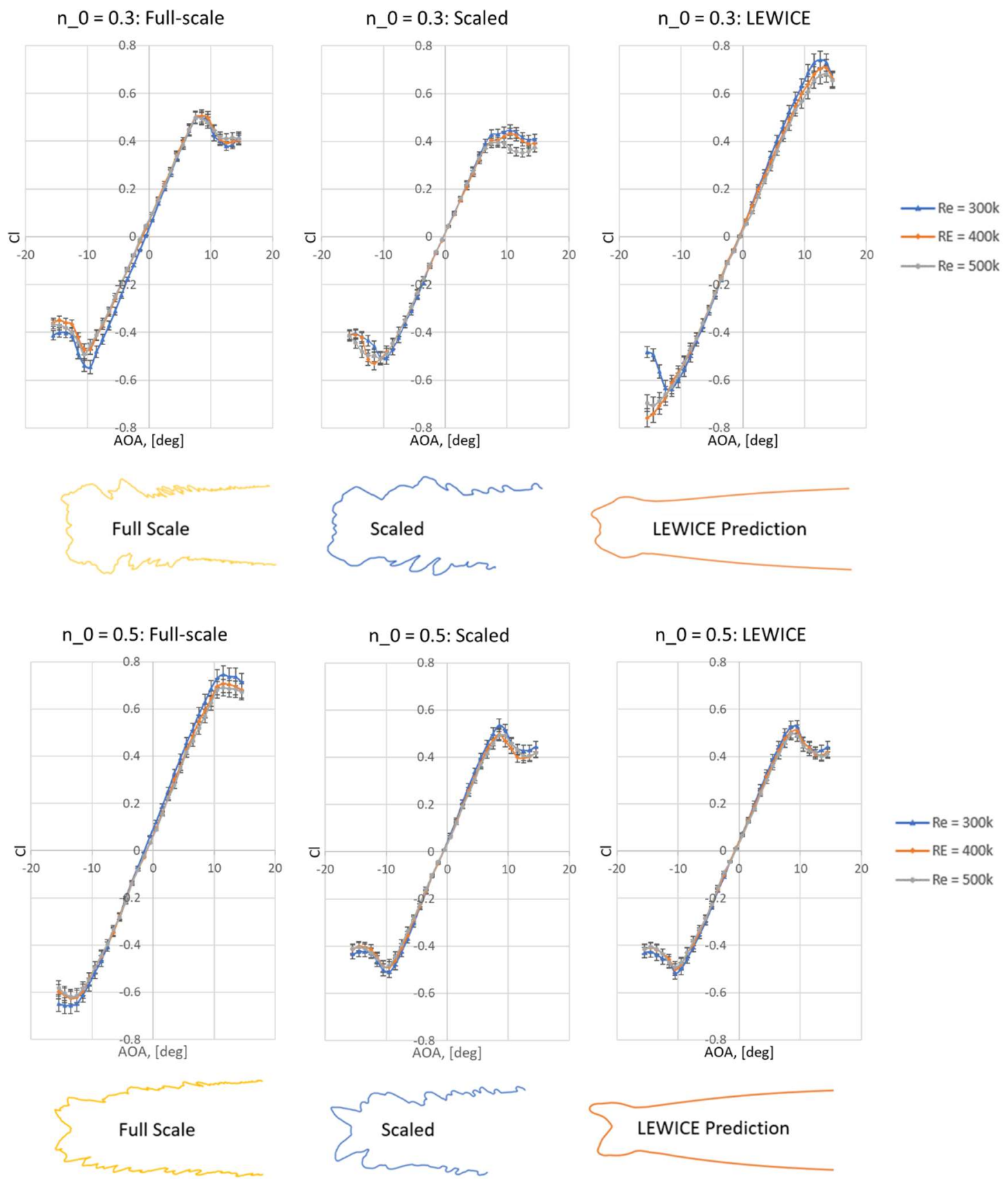

Figure 31: Lift coefficient variation for each ice accretion cases as a function of Reynolds number 

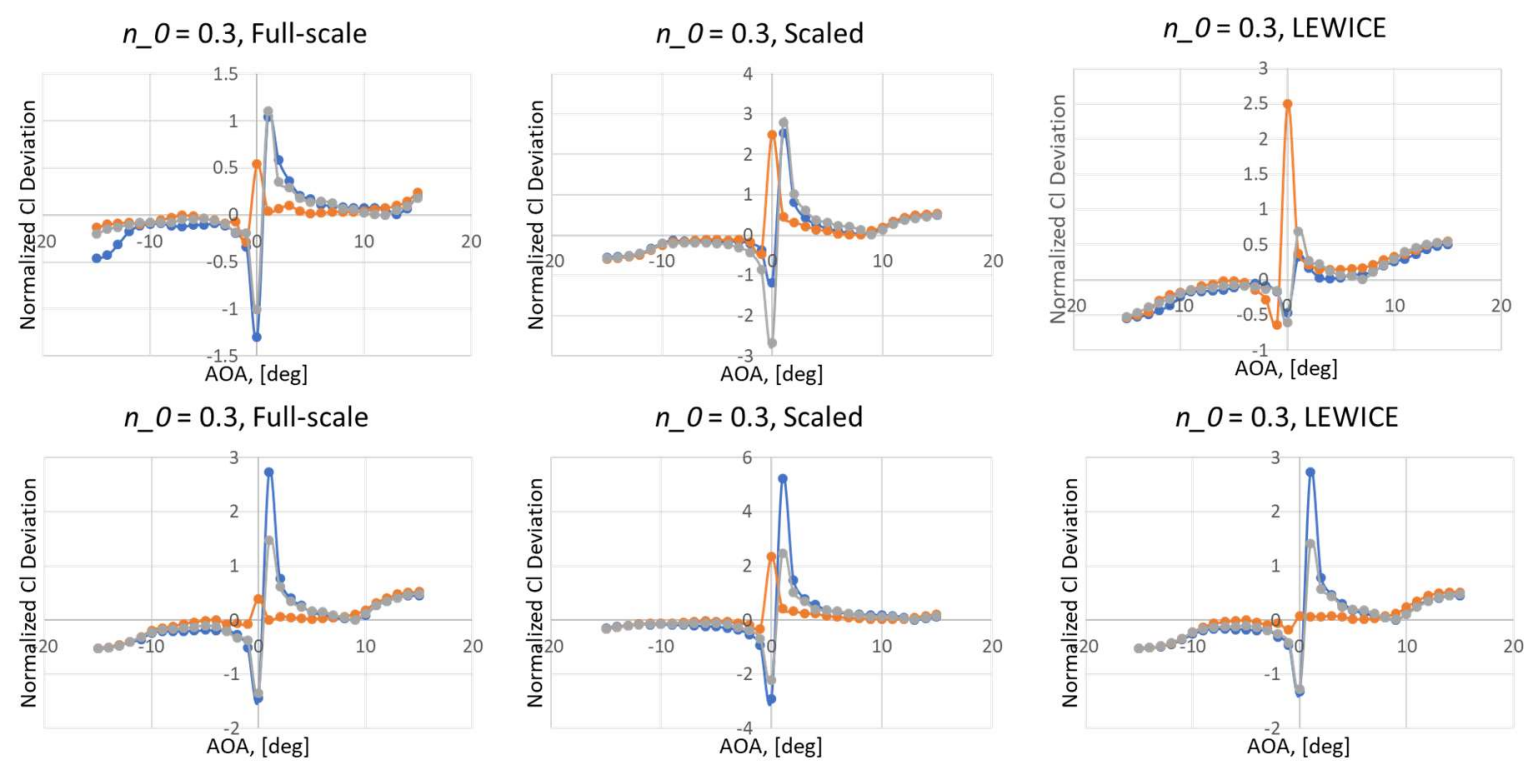

$\rightarrow 300 k$

$\rightarrow 400 k$

$\rightarrow-500 k$

Figure 32: Normalized Lift coefficient variation for each ice accretion cases

The highest variation between the different Reynolds numbers occurs at the extremely low angles of attack between $-2^{\circ}$ and $2^{\circ}$. This occurs due to the values of $C_{l}$ being small in magnitude, therefore any minute changes results in a more significant deviation from the baseline. However, the deviations for the higher angles of attack outside this range are highly consistent for each of the ice accretions across the Reynolds numbers examined, with exception to the $n_{0}=0.3$ full-scale ice shape. The deviation seen from the $n_{0}=0.3$ full-scale ice occurs at high negative angles of attack past $-12^{\circ}$. Therefore, based on the Reynolds number insensitivity seen between $-12^{\circ}$ and $12^{\circ}$ angle of attack, PIV was performed at a single Reynolds number of 300,000 across this angle of attack sweep. The discussed results presented throughout the rest of the paper, including the force-based experimental results, correspond to a Reynolds number of 300,000, corresponding to a free stream test section velocity of $22.06 \mathrm{~m} / \mathrm{s}$.

\subsection{Aerodynamic Coefficient of Lift}

This section focuses on the associated aerodynamic penalties with respect to lift. As discussed in Chapter 3, the experimental setup for the force-based testing was unable to produce 
an ideal two-dimensional flow. Therefore, the AR effect was determined by comparing the linear portion of the NACA 0012 lift curve slope, see Figure 33, of 3.64, to the Helmbold low aspect ratio straight-wing equation, Equation 34.

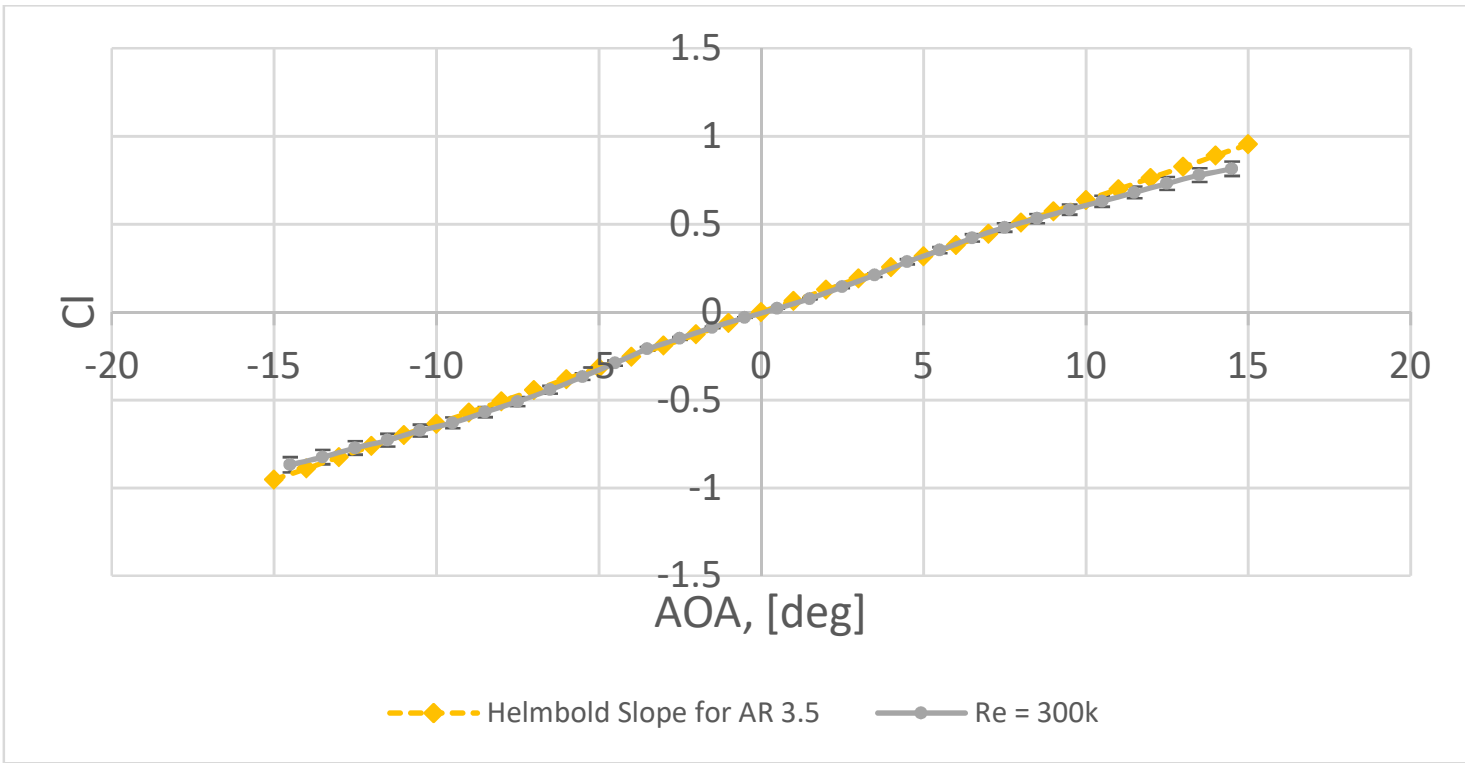

Figure 33: Baseline NACA lift coefficient curve, $R e=\mathbf{3 0 0 , 0 0 0}$

The Helmbold equation determined the experimental setup produced conditions representative of a wing with an AR of $\sim 3.5$. Though two-dimensional aerodynamics were not created for the force-based experiments, the setup was consistent for all models tested; therefore, the results are able to be directly compared.

\subsection{1 $n_{0}=0.3$ Ice accretions}

Figure 34 includes the comparisons of the lift coefficients for the $n_{0}=0.3$ ice shapes. The ice shape geometries are included in the figures for reference. As shown, the scaled ice shape is able to reproduce a similar trend at lower angles of attack in the lift coefficient when compared to the full-scale ice shape. However, differences occur at higher angles of attack with the scaled ice shape producing a lower maximum lift coefficient, $C_{l, \max }$. The difference between the scaled and full-scale ice shapes is easier seen at the high positive angles of attack, where the scaled ice shape 
has a more gradual stall. The two ice shapes share a similar stall angle, but the full-scale ice shape produces a higher $C_{l, \max }$. The difference in the high positive angles of attack stall trends could be attributed to the large, more aft positioned feather region ice accretion on the upper surface of the scaled ice shape.

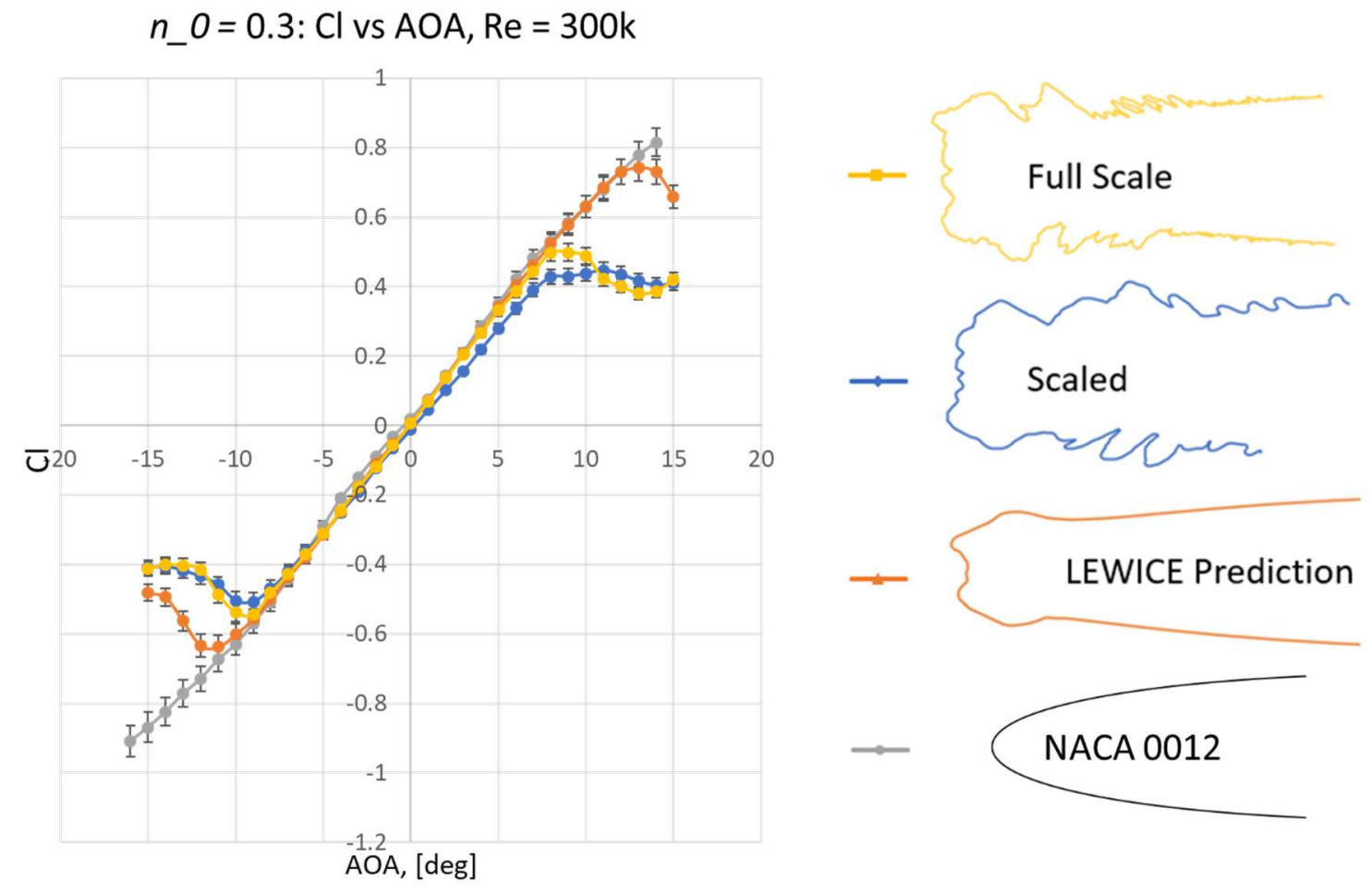

Figure 34: Lift coefficient comparison for $\boldsymbol{n}_{0}=0.3$ ice accretion cases

The LEWICE predicted ice accretion is also able to reproduce a similar lift coefficient trend at lower angles of attack. However, it greatly differs from the full-scale at high angles of attack, stalling significantly later than either the full-scale of scaled ice shapes. The LEWICE accretion stalls around $-13^{\circ}$ and $13^{\circ}$ compared to roughly $-9^{\circ}$ and $9^{\circ}$ of the full-scale and scaled ice accretions. The LEWICE ice shape's lower and upper horns are both significantly shorter than the full-scale ice shape. The cause of stall delay will be discussed with the context of the PIV results in the next chapter. However, it can be hypothesized that the delayed stall is a result of the flow not fully-separating until higher angles of attack when compared to the other two cases. 


\subsection{2 $n_{0}=0.5$ Ice accretions}

Figure 35 includes the comparisons of the lift coefficients for the $n_{0}=0.5$ ice shapes. The ice shape geometries are included in the figures for reference. As shown, the scaled ice shape is able to reproduce a similar trend in the lift coefficient to the full-scale at low angles of attack. The scaled ice shape greatly differs from the full-scale ice shape at high angles of attack, showing a significantly earlier stall and lower $C_{l, \max }$ than the full-scale ice shape. The scaled ice shape stalls around $-9^{\circ}$ and $9^{\circ}$, whereas the full-scale ice shape stalls, more gradually, around $-12^{\circ}$ and $13^{\circ}$. The full-scale also has a $C_{l, \max } \sim 0.1$ greater in magnitude than the scaled ice shape. The earlier stall of the scaled ice shape could be attributed to its large feather regions ice accretions. The horn shapes are very similar between the scaled and full-scale, with the upper horn being slightly taller in height, but the full-scale has significantly more minute feather region ice accretions.

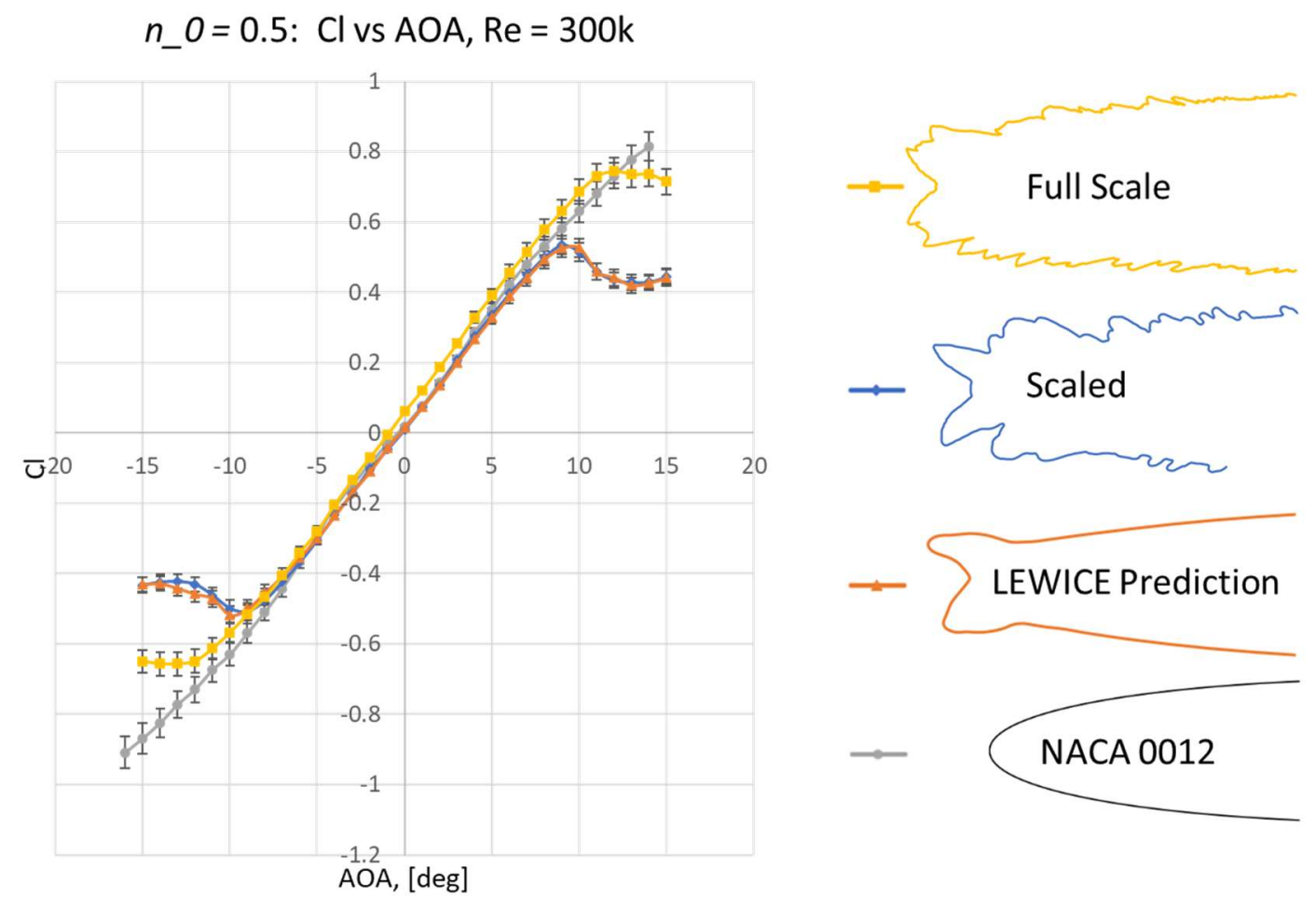

Figure 35: Lift coefficient comparison for $n_{0}=0.5$ ice accretion cases

The LEWICE ice shape is also able to reproduce a similar trend to the full-scale at low angles of attack, but differs significantly at high angles of attack. The LEWICE lift coefficient trend 
is almost identical to that of the scaled ice shape, showing a very similar stall around $-9^{\circ}$ and $9^{\circ}$. The $C_{l, \max }$ of the LEWICE ice shape is also $\sim 0.1$ less in magnitude than that of the full-scale ice shape. This can be attributed to the slightly greater horn angle of the lower and upper horns of the LEWICE ice shape compared to both the full-scale and scaled ice shapes (Figure 14). The horn height of the lower LEWICE horn is very similar to the full-scale, but the larger horn angle, which the associated aerodynamics are also highly sensitive to, causes an early stall in comparison to the full-scale ice shape.

\subsection{Aerodynamic Coefficient of Drag}

The average streamwise U-velocity profiles in the near wake determined through the momentum deficit principle, are discussed in the section. Following the discussion of the near wake profiles, the drag coefficient estimated from the momentum deficit principle is discussed. The differences in the drag coefficient at different angles of attack are directly shown through the velocity profiles for each of the ice shapes. Along with the momentum deficit estimated drag coefficient, the drag coefficient determined from the force-based experimentation is also discussed. The drag coefficient magnitude varies between the force-based experimentation and momentum deficit estimation due to the difference in the three-dimensional effects present based on each setup, but the trends shown are highly comparable. The trends in the momentum deficit estimated drag coefficients were also the same as those seen in the force-based drag coefficients.

The wake velocity profiles for an angle of attack range from $-12^{\circ}$ to $12^{\circ}$ in increments of $4^{0}$, along with the drag coefficient versus angle of attack curves for each of the ice shapes is presented in this section. The ice accretions collected at $n_{0}=0.3$ and $n_{0}=0.5$ are discussed individually.

\subsection{1 $n_{0}=0.3$ Ice accretions}

The velocity profiles for angle of attack ranging from $-12^{\circ}$ to $12^{\circ}$ for the $n_{0}=0.3$ ice accretions are presented in Figure 36, followed by the momentum deficit estimated drag coefficients and the force-based determined drag coefficients in Figure 37. 

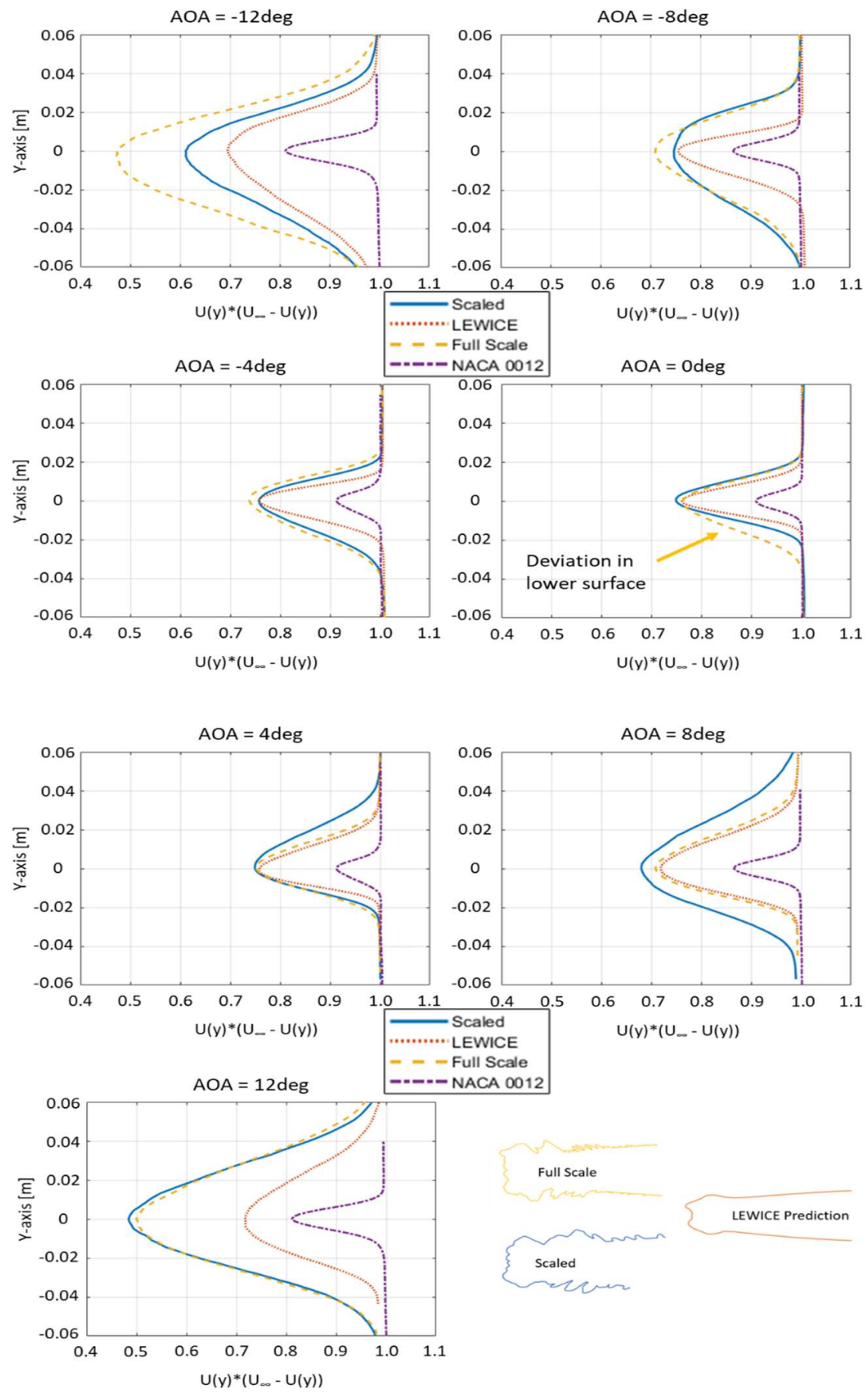

Figure 36: Near wake mean $U$-velocity profiles for $\boldsymbol{n}_{0}=0.3$ ice accretion cases

At $0^{\circ}$ angles of attack, wake profile behind the scaled ice accretion matches relatively well with the full-scale. However, the wake profile is clearly asymmetric except for the baseline NACA 0012. As annotated in Figure 36, the wake profile associated with the lower surface of the wing shows higher deviations between the full-scale and the scaled model when compared to the wake 
profile associated with the upper surface. This is due to the taller lower horn of the full-scale ice accretion. Throughout the negative angles of attack, the momentum deficit of the full-scale ice accretion is greater than that of the other two ice shapes. The taller lower horn of the full-scale ice shape could be responsible for this deviation through causing a larger region of separated flow.

As annotated in Figure 36 for the $-4^{0}$ angle of attack, the wake profile associated with the upper surface of the scaled ice shape deviates from the full-scale ice shape. The momentum deficit of the scaled ice shape continues to be greater than the full-scale until stall is reached at $12^{\circ}$. This could be due to the large, aft positioned feather region ice accretion on the upper surface of the scaled ice shape causing significant flow separation.

The LEWICE ice shape greatly differs from the full-scale ice shape from $-12^{\circ}$ through $0^{\circ}$, showing significantly less momentum deficit than the full-scale ice shape. The same deviation at $0^{\circ}$ between the scaled and full-scale ice shapes can be seen between the LEWICE and full-scale ice shape. The deviation at $0^{0}$ is due to the taller lower horn of the full-scale ice shape and the significantly smaller lower horn of the LEWICE ice shape is responsible for the less momentum deficit throughout the negative angles of attack.

The upper horn of the LEWICE ice shape also differs greatly in geometry, especially in height, from the upper horn of the full-scale ice shape. At $4^{0}$ angle of attack, the full-scale ice shape deviates from the LEWICE with respect to the lower surface. At $8^{\circ}$ angle of attack, the wake profile produced by the LEWICE ice shape coincides well with the full-scale ice shape, with small deviation. This similarity does not continue at $12^{\circ}$, where stall is reached by the full-scale ice shape. At $12^{\circ}$, the flow on the LEWICE model is still attached, as shown by the lift coefficients, creating significantly less momentum deficit than the upper surface separated flow of the full-scale ice shape.

The momentum deficit principle estimated drag coefficient and the force-based drag coefficient curves for the $n_{0}=0.3$ ice accretions are presented in Figure 37. Ice shape geometry profiles are provided for reference. The aerodynamic penalty of increased drag, typical of ice 
accretion, is clearly shown by all three ice shapes, when compared to the baseline NACA 0012 airfoil.

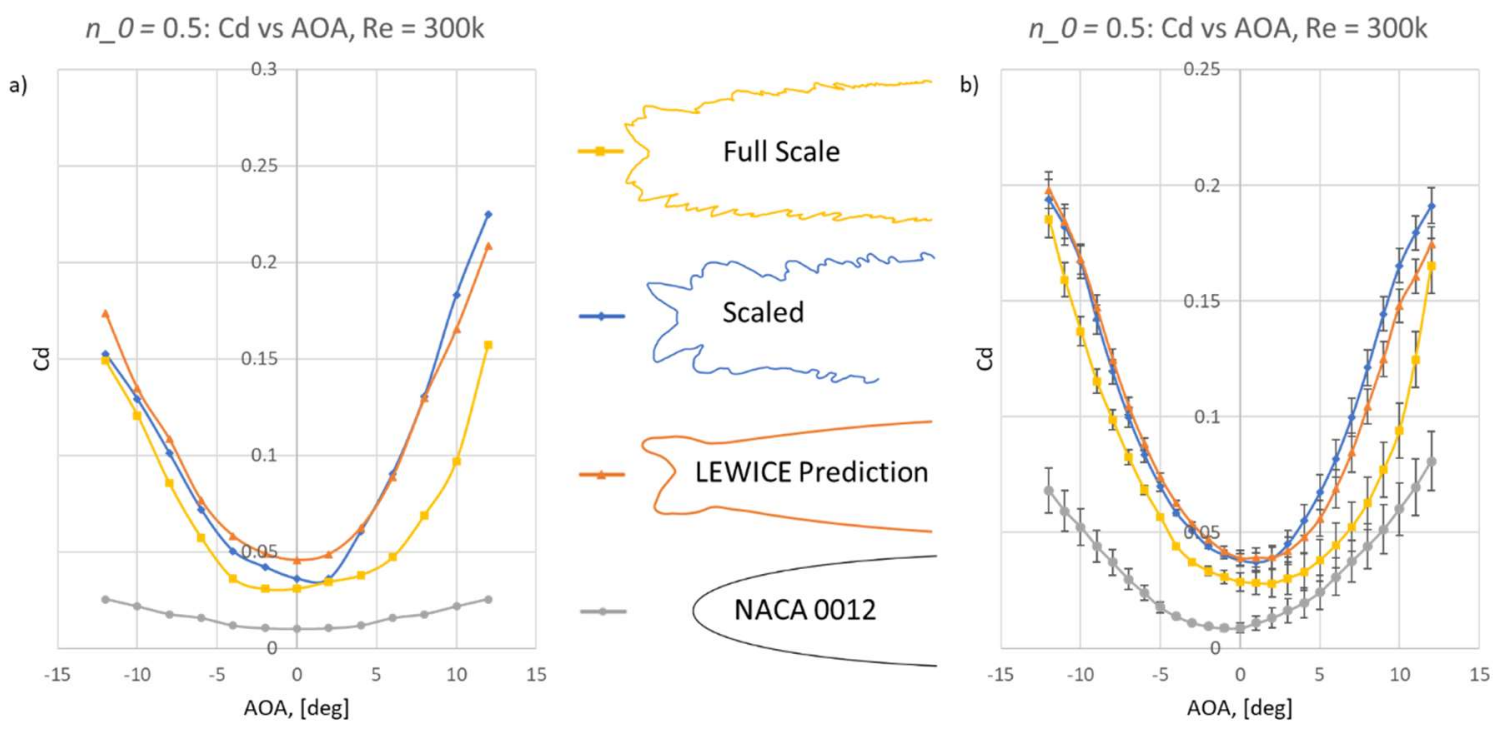

Figure 37: Drag coefficient comparison obtained from a) momentum deficit principle b) force-based testing for $\boldsymbol{n}_{0}=\mathbf{0 . 3}$ ice accretion cases

As shown, the trends between drag coefficient determined from force-based testing and momentum deficit correlate well. The drag coefficient variation from the force-based testing shows slightly higher magnitude than the momentum deficit results. This difference can be attributed due to the nature of the two testing methods. The drag coefficient present in Figure 37 presents the sectional drag which is better estimated by the momentum deficit principle than the force-based testing. This is because the drag coefficient from the force-based testing includes aerodynamic interference between the wing model and the splitter plate and the associated three-dimensional effects which increases the magnitude of the drag coefficient seen. Albeit the higher magnitude both the drag coefficient results show very similar trends for the different ice shapes.

The full-scale and scaled ice shapes differ in the same angle of attack regions as shown by the near wake streamwise velocity profiles in Figure 36. The primary areas where the drag coefficient deviate between the full-scale and scale ice shapes are $0^{\circ}$ through $-4^{\circ}$ and $4^{\circ}$ through $10^{\circ}$. The full-scale ice shape drag coefficient is greater in magnitude than the scaled ice shape from $0^{0}$ 
through $-4^{0}$. This is most likely due to the taller lower horn of the full-scale ice shape and is corroborated by the lower surface deviation shown by the $0^{0}$ velocity profiles in Figure 36 . As for $4^{0}$ through $10^{\circ}$, the scaled ice shape produces greater drag coefficients than the full-scale ice shape. This is due to the upper surface deviation shown by the $4^{0}$ velocity profiles in Figure 36 . The fullscale and scaled ice shapes have similar upper horn geometries; therefore, it is suspected that the large, aft positioned upper surface feather region ice accretion of the scaled ice shape is responsible for this deviation.

The LEWICE ice shape is unable to reproduce drag coefficients similar to the full-scale ice shape throughout the range of angle of attack. This is corroborated by the significantly less momentum deficit expressed by the near wake velocity profiles in Figure 36. The drag coefficient of the LEWICE is the closest to the full-scale drag coefficient at positive midrange angles of attack, which is corroborated by the similar velocity wake profiles in Figure 36. The upper and lower horns of the LEWICE ice shape are both significantly smaller in size comparatively to the full-scale ice shape. This in turn leads to lower drag coefficients across the whole range and the delayed stall seen in the previous section. From the lift and drag coefficient variations, the LEWICE ice shape's inability to reproduce the aerodynamic characteristics with that of the full-scale can be confirmed.

\subsection{2 $n_{0}=0.5$ Ice accretions}

The velocity profiles for angles of attack ranging from $-12^{\circ}$ to $12^{\circ}$ for the $n_{0}=0.5$ ice accretions are presented in Figure 38, followed by the momentum deficit estimated drag coefficients and the force-based determined drag coefficients in Figure 39. 

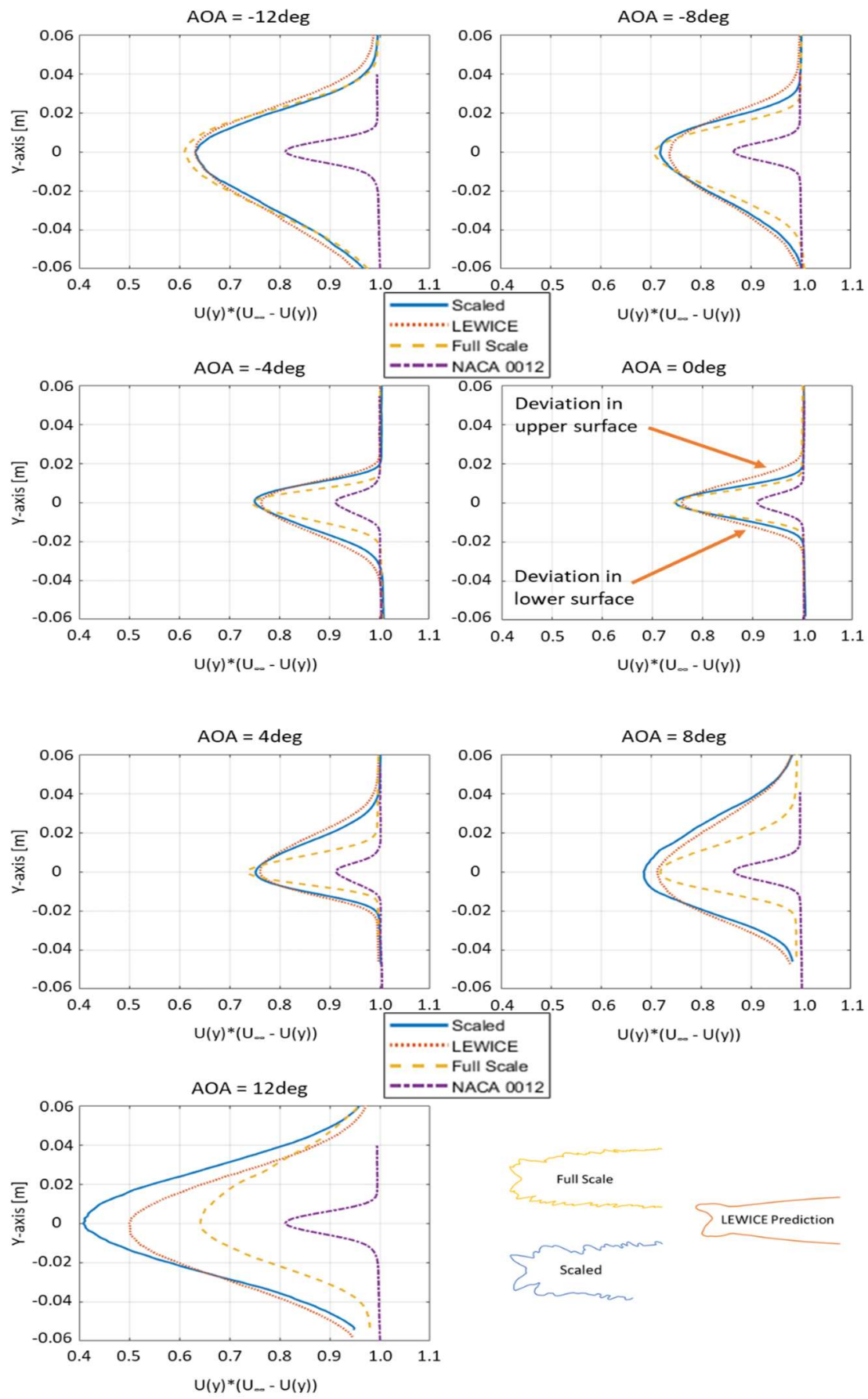

Figure 38: Near wake mean U-velocity profiles $\boldsymbol{n}_{0}=0.5$ ice accretion cases

At $0^{\circ}$, the full-scale and scaled ice shapes wake velocity profiles compare well, showing minimal deviation in the wake associated with both the upper and lower surface. However, a clear difference in the wake width is seen at $-4^{0}$, where the wake width of the scaled ice shape is wider than the full-scale ice shape. The lower horn is slightly taller on the scaled ice shape compared to 
the full-scale and could be causing this deviation at low negative angles of attack. There is also some deviation at $-8^{0}$, where the scaled ice shape has a wider wake width, but the deviation is less than it was at $-4^{0}$. At $-12^{0}$, the wake profiles are highly similar, with minimal deviation.

At positive angles of attack, the scaled ice shape consistently deviates from the full-scale ice, showing greater momentum loss. The deviation between the ice shapes increases as the angle of attack increases. This is due to the upper horn of the scaled ice shape being taller than the fullscale upper horn and having a pronounced second horn structure formed in the feather region that is significantly taller than the main upper horn. The greatest difference between the momentum deficit occurs at $12^{\circ}$, where the flow is fully separated from the upper surface of the scaled ice shape and the full-scale ice shape has yet to stall.

As annotated in Figure 38, the wake profile associated with both the upper and lower surfaces of the LEWICE ice shape deviates from the full-scale. The LEWICE ice shape wake profiles vary from the full-scale in the same manner as the scale ice shape though the negative angles of attack, but with a wider wake profile than the scaled ice shape. At $-12^{\circ}$ the momentum deficit is highly comparable between the LEWICE and full-scale ice shapes. Though the lower horns of the LEWICE and full-scale ice shapes are very similar in geometry, the feather region ice accretions on the full-scale produce a more gradual change in the geometry. Whereas, the absence of the feather region with respect to the LEWICE ice shape could lead to a region of adverse pressure, causing greater separation than the full-scale ice shape.

At positive angles of attack, the LEWICE shows significantly greater momentum deficit than the full-scale ice shape. In the same manner, the difference in the momentum deficit increases with increasing positive angle of attack, though deviating less than the scaled ice shape. This can be attributed to the taller upper horn and greater horn angle of the LEWICE ice shape. The greatest deviation is again shown at $12^{\circ}$, where the LEWICE ice shape is post stall and the flow is fully separated from the upper surface. The full-scale ice shape is still not stalled at this angle of attack which causes the large discrepancy in the wake profiles at $12^{\circ}$. 
The momentum deficit principle estimated drag coefficient and the force-based drag coefficient curves for the $n_{0}=0.5$ ice accretions are presented in Figure 39. Ice shape geometry profiles are provided for reference. The aerodynamic penalty of increased drag, typical of ice accretion, is clearly shown by all three ice shapes, when compared to the baseline NACA 0012 airfoil.
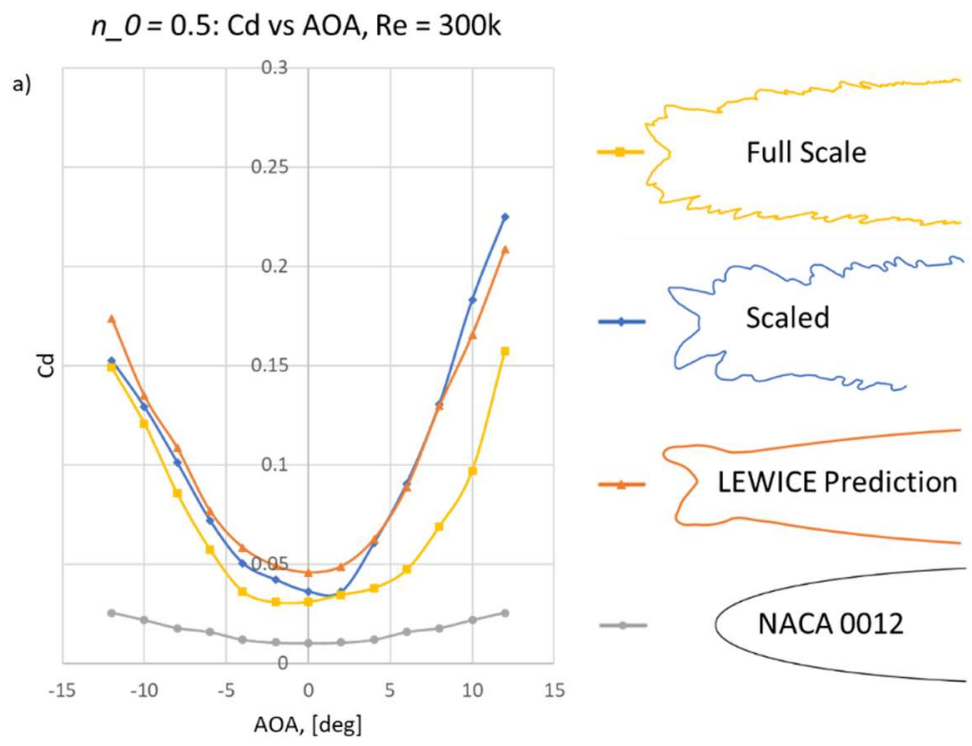

b)

$n \_0=0.5:$ Cd vs AOA, $\operatorname{Re}=300 \mathrm{k}$

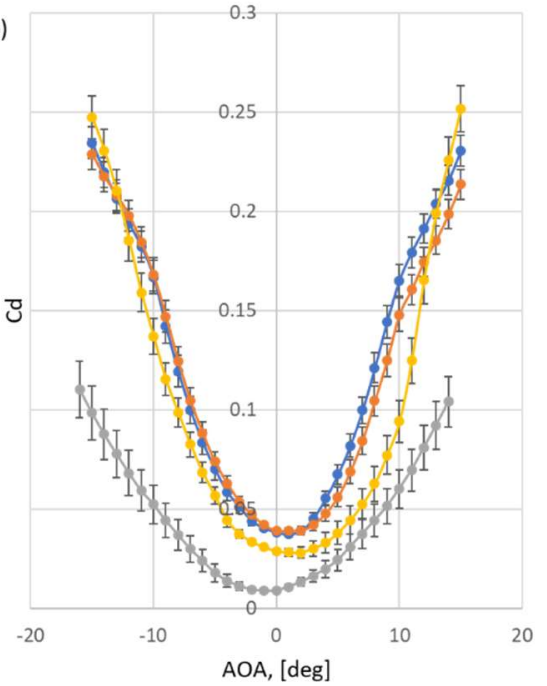

Figure 39: Drag coefficient comparison obtained from a) momentum deficit principle b) force-based testing for $\boldsymbol{n}_{0}=0.51$ ice accretion cases

As shown, the trends between drag coefficient determine from force-based testing and momentum deficit correlate well. The drag coefficients associated with the scaled ice shape are consistently greater than the full-scale across the whole range of angles of attack, being most similar at high negative angles of attack and between $0^{\circ}$ and $2^{\circ}$. The drag differentiation shows that though the lift coefficient curves are similar at low and midrange angles of attack, the scaled ice shape still differs from the full-scale across the whole range. The slightly taller lower horn is responsible for the slightly higher drag coefficients at negative angles of attack and the large pronounced upper second horn feature and the taller upper horn of the scaled ice shape are responsible for the significantly higher drag at positive angles of attack. 
The LEWICE ice shape deviates from the full-scale even more so than scaled ice shape, having consistently higher drag coefficients throughout the whole angle of attack range. The LEWICE is most similar to the full-scale ice accretion at high negative angles of attack, where the similar lower horns create similar momentum deficit in the wake. At positive angles of attack, the LEWICE ice shape deviates from the full-scale at a similar magnitude as the scaled ice shape. This can be attributed to the taller upper horn with a greater horn angle when compared to the full-scale ice accretion.

\subsection{Summary of Chapter 4}

The force-based experimentation and momentum deficit analysis showed inconsistency in the scaling methods ability to generate an ice shape that preserves the aerodynamics associated with the full-scale ice shape. The analysis also showed that the LEWICE software was unable to predict an ice shape which is aerodynamically characteristic of the full-scale ice shape under SLD conditions. In the $n_{0}=0.3$ ice accretions, the scaled ice shape showed similar aerodynamic coefficients and trends when compared to the full-scale ice shape as observed in coefficient of lift and in coefficient of drag. The drag coefficient deviated at midrange positive angles of attack ( $4^{0}$ through $10^{\circ}$ ), where the drag coefficient of the scaled ice shape was greater than that of the fullscale. This deviation could be attributed to upper surface large feather structure of the scaled ice shape that is positioned significantly aft of the horn structure. The LEWICE ice shape collected at a $n_{0}=0.3$ was not characteristic of the full-scale ice shape in either lift or drag coefficient. This could easily be attributed to the overall geometric differences between the LEWICE and full-scale ice shape.

As for the ice shapes collected at a $n_{0}=0.5$, the LEWICE ice shape and the scaled ice shape showed similar aerodynamic coefficients and trends when compared to the full-scale ice accretion. In the $n_{0}=0.5$ ice accretions, the scaled and LEWICE ice shapes compared better to the full-scale at negative angles of attack than at positive angles of attack, as observed in the coefficient of drag. This was due to the difference in horn height of the upper horn structure, 
whereas the lower horn structures were more geometrically similar across the three ice accretions. The scaled ice shape not being characteristic of the full-scale could be due to the icing conditions and the resulting horn ice shapes those conditions generate. The major difference between the two icing conditions was the $n_{o}$, which affects the temperature, as specified in Figure 10 . The temperatures at which the ice shapes were collected could suggest a temperature and/or stagnation freezing fraction dependency of the importance of the feather region and of the scaling method's usability for SLD conditions. 


\section{CHAPTER V}

\section{PIV FLOW FIELD ANALYSIS}

The flow field analyses of the different ice shapes, performed through particle image velocimetry (PIV), are discussed in this chapter. The flow analysis is separated into three sections based on the flow field parameter being studied. The three parameters studied are the streamwise (U-component) of velocity, the vorticity, and the Reynolds stress. The parameters are compared for

both the on-body and free shear PIV simultaneously. The discussion of the different parameters is followed by a summarization of the overall flow field aerodynamic preservation.

\subsection{PIV Flow Field Analysis Results Overview}

The U-component of velocity allows for the study of the flow separation, presence or lack thereof a separation bubble, and flow reattachment. The vorticity allows for the study of the angular rotation of the flow and the areas of total pressure loss. Lastly, the Reynolds stress allows for the study of momentum transport due to turbulence and indicates the regions with highly correlated fluctuating velocity components. For each of these parameters, the on-body and free shear layer flow fields for angle of attack $-12^{\circ},-6^{0}, 0^{\circ}, 6^{\circ}$, and $12^{\circ}$ is presented. Each of these angles of attack highlights a different region of the lift curve slope. The positive and negative $12^{\circ}$ angles of attack allow to the study of flow physics in the near stall region for each of the ice shapes. The positive and negative $6^{\circ}$ angles of attack allow for the study of the ice shapes in the linear region of the lift curve slope and the $0^{\circ}$ angle of attack allows for deviations at a neutral position to be evaluated.

\subsection{Mean U-velocity Component}

\subsection{1 $n_{0}=0.3$ Ice Accretions Results}

The normalized U-component velocity contours for the ice accretions collected at a $n_{0}=$ 0.3 is shown in Figure 40. It is important to note that the contour map range is different between the on-body and free shear layer contours. 

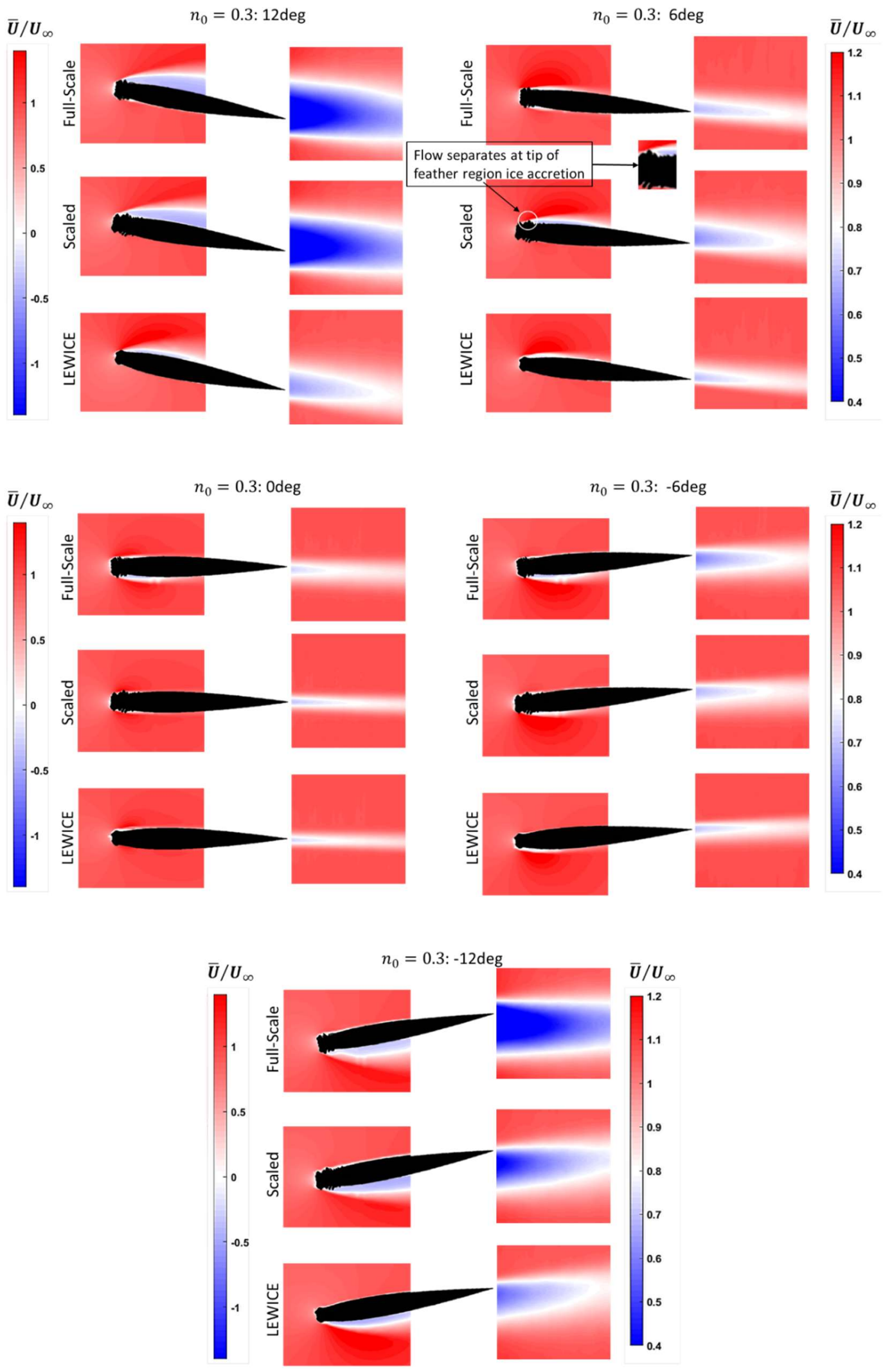

Figure 40: Normalized U-velocity contours for $n_{0}=0.3$ ice shapes 
The contour range for the on-body contours is provided on the left-hand side of the figure and the contour range for the free shear layer is provided on the right-hand side of the figure. The velocity is normalized by the freestream velocity of $22.06 \mathrm{~m} / \mathrm{s}$. The on-body streamlines for each of the ice shapes are shown in Figure 41, where the presence of a separation bubble and complete flow separation can be observed.
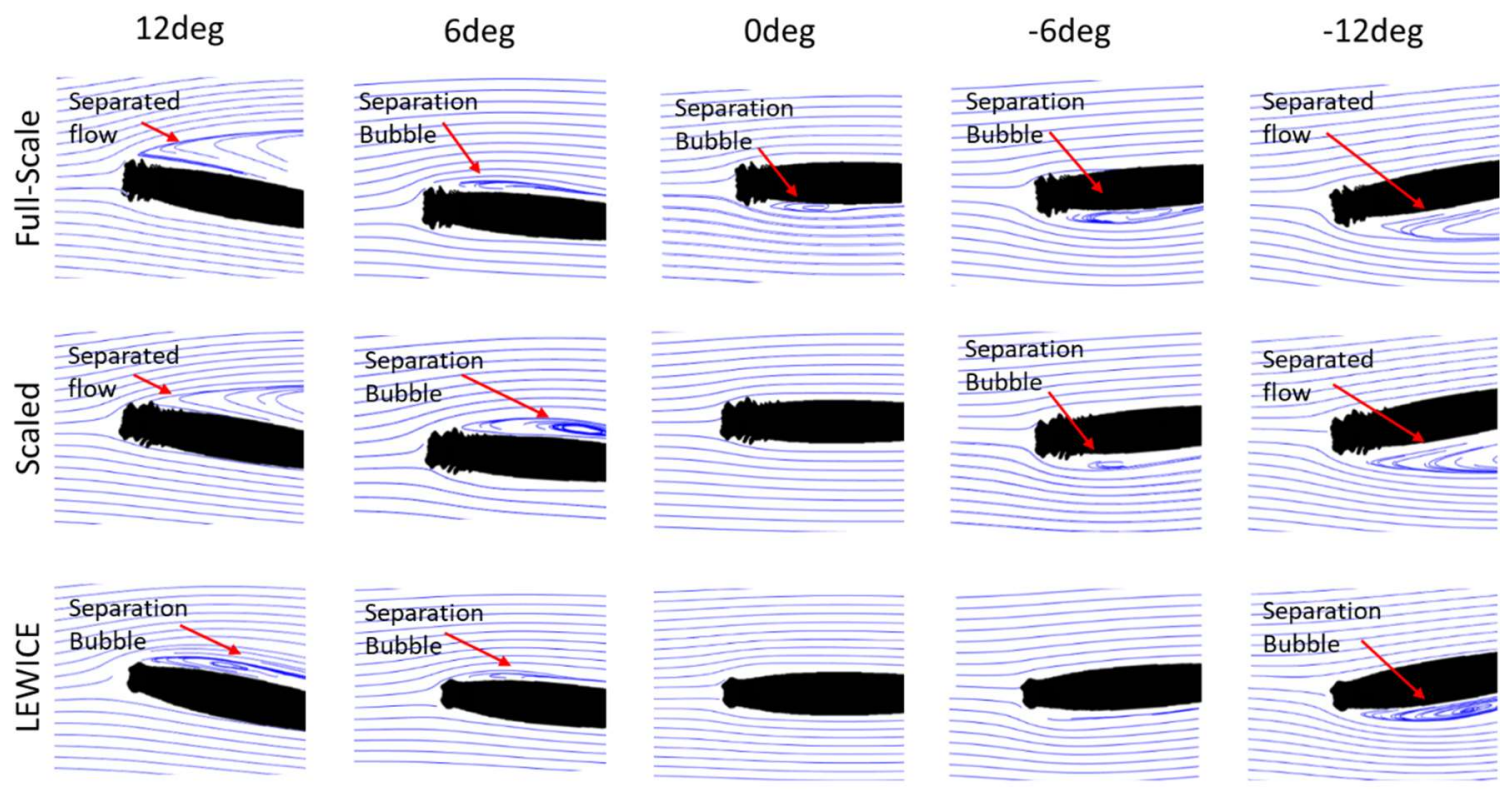

Figure 41: On-body streamlines for $n_{0}=0.3$ ice shapes

At the positive $12^{\circ}$ case, the flow is separated for the full-scale and scaled ice accretions, but not the LEWICE ice shape, as annotated in Figure 41. This trend correlates with the force-based results where the full-scale and scaled ice shapes stalled prior to $12^{\circ}$, indicated by a sudden drop in the lift coefficient. This same sudden drop off was not observed for the LEWICE ice shape. A clear separation bubble is seen on the upper surface of the LEWICE ice shape, annotated in Figure 41, and is responsible for the flow remaining attached at $12^{\circ}$. The near wake flow physics show a thicker wake width in the case of the full-scale and scale ice accretion compared to the LEWICE ice accretion. This is once again corroborated by the significant increase in the drag coefficient in the full-scale and scaled model when compared to the LEWICE model, as shown in Figure 39. 
At a positive $6^{0}$ angle of attack, the scaled and full-scale ice shapes deviate from one another. Though they both show a separation bubble on the upper surface, the separation bubble is significantly larger for the scaled ice shape, as seen in Figure 41. This causes flow reattachment to occur significantly further aft on the scaled ice shape airfoil causing a thicker boundary layer. The wake physics show a thicker wake width for the scaled ice shape, when compared to the full-scale and LEWICE structures. This difference between the full-scale and scaled ice shapes for this region of angle of attack was also shown by the momentum deficit and drag coefficient results in Figures 36 and 37 . At $6^{\circ}$, the drag coefficient of the scaled ice shape is greater than that of the full-scale or LEWICE ice shapes. The scaled and full-scale upper horns are of similar geometry, suggesting the large feather region structure present on the upper surface of the scaled ice accretion may be affecting the flow field. As annotated in Figure 40, this was shown to be the case, as separation occurs at the tip of the feather region ice accretion. The LEWICE ice shape creates an upper surface separation bubble similar in size to the full-scale ice shape, but neither the scaled or LEWICE ice shapes show separation on the lower surface. The full-scale ice shape, on the other-hand, does have a small region of separation on the lower surface, resulting in a slightly higher drag coefficient than the LEWICE ice shape, as shown in Figure 37.

Just like at $6^{0}$, the full-scale ice shape has a larger separation bubble on the lower surface than either the scaled or LEWICE structures at $0^{\circ}$, as seen in Figure 41. The larger separation bubble is caused by the taller lower horn of the full-scale ice shape. The resultant increase in the drag coefficient associated with the larger wake for the full-scale ice shape when compared to the other two cases is, once again, strongly corroborated by the drag coefficient variation in Figure 37.

Similar to the $0^{\circ}$ case, the larger lower surface separation bubble continues to persist at $-6^{0}$ angle of attack with resect to the full-scale ice shape, as shown in Figure 41. This results in a slightly thicker wake width, as shown in the wake contours, when compared to the scaled and LEWICE ice shapes. The resultant increase in wake thickness once again manifests as increase in the drag 
coefficient of the full-scale ice shape, as corroborated by drag coefficient deviation from the momentum deficit results in Figure 37.

Though the flow on both the full-scale and scaled ice shapes is fully separated at $-12^{\circ}$, the momentum lost in the wake is stronger in the full-scale ice shape. Flow on the lower surface of the LEWICE ice shape has not yet separated, as shown by the streamlines in Figure 41. The large separation bubble results in significantly less momentum loss in the wake, supported by the significantly lower drag coefficient than the other two ice shapes at $-12^{\circ}$ angle of attack in Figure 37.

\subsection{2 $n_{0}=0.3$ Ice Accretions Summary}

In terms of reproducing the streamwise mean velocity component of the full-scale ice shape, the scaled ice shape was better than the LEWICE ice shape at a stagnation freezing fraction of 0.3 . At $6^{0}$, the upper surface separation bubble formed by the scaled ice shape was larger than the full-scale ice shape and was found to be due the large feather region structure of the scaled ice shape that is considerably aft of the frontal upper horn. Deviation also occurred at the negative angles of attack, where the taller lower horn of the full-scale ice shape created a larger separation bubble and a corresponding wake with a greater momentum deficit and larger wake width. than the scaled and LEWICE ice shapes. As for the LEWICE ice shape, it deviated from the full-scale across the entire range of angles of attack and was especially noticeable at $-12^{\circ}$ and $12^{\circ}$, where the flow was not full-separated. This was expected based on the force-based and momentum deficit results.

\subsection{3 $n_{0}=0.5$ Ice Accretions Results}

Figure 42 includes the normalized U-component velocity contours for the ice accretions collected at a $n_{0}=0.5$. It is once again important to note that the contour map range differed between the on-body and free shear layer contours. 

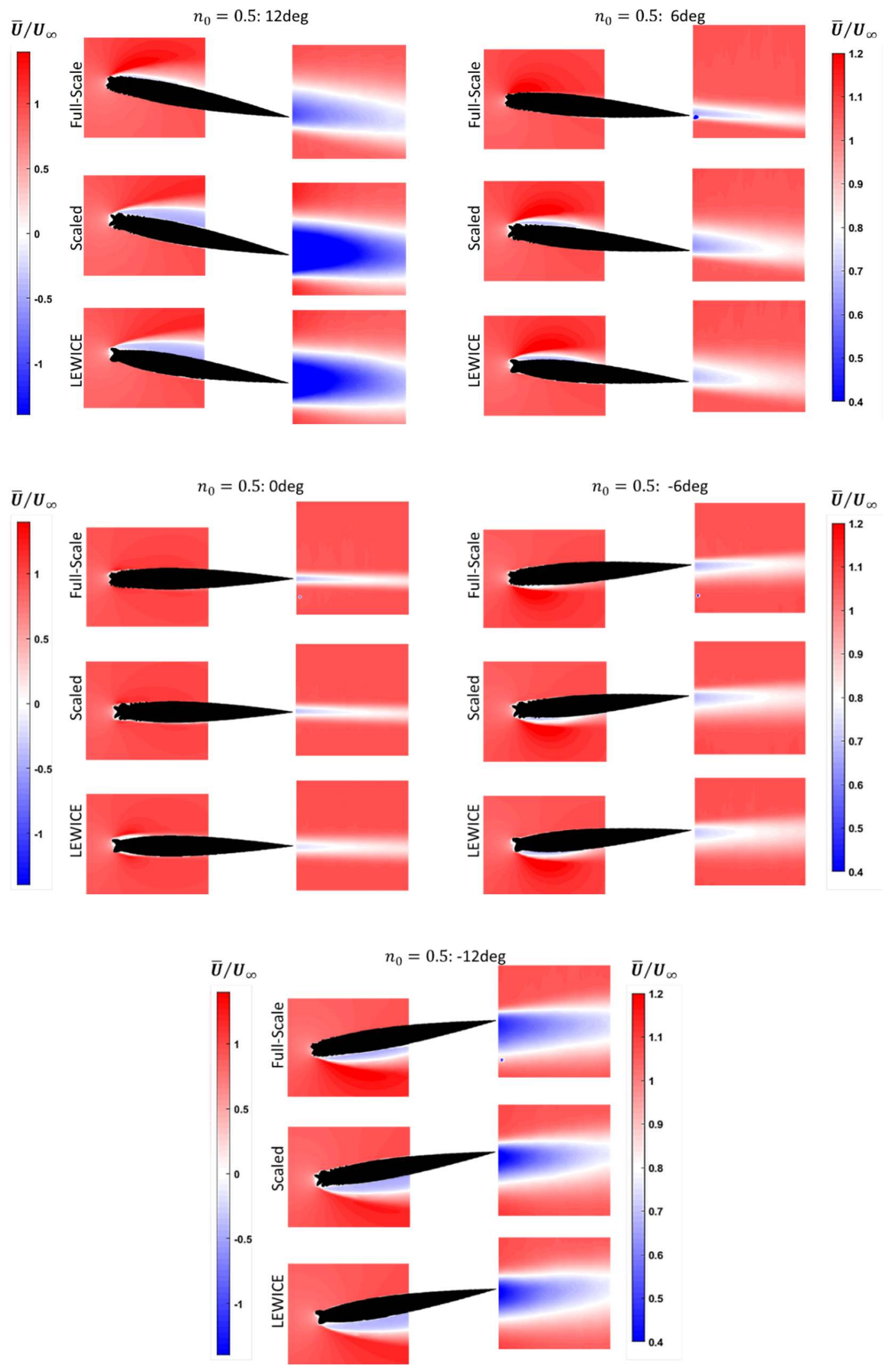

Figure 42: Normalized U-velocity contours for $n_{0}=0.5$ ice shapes 
The contour range for the on-body contours is provided on the left-hand side of the figure and the contour range for the free shear layer is provided on the right-hand side of the figure. The velocity is normalized by the freestream velocity of $22.06 \mathrm{~m} / \mathrm{s}$. The on-body streamlines for each of the ice shapes are shown in Figure 43. The streamlines allowed the presence of a separation bubble versus complete flow separation to be determined.
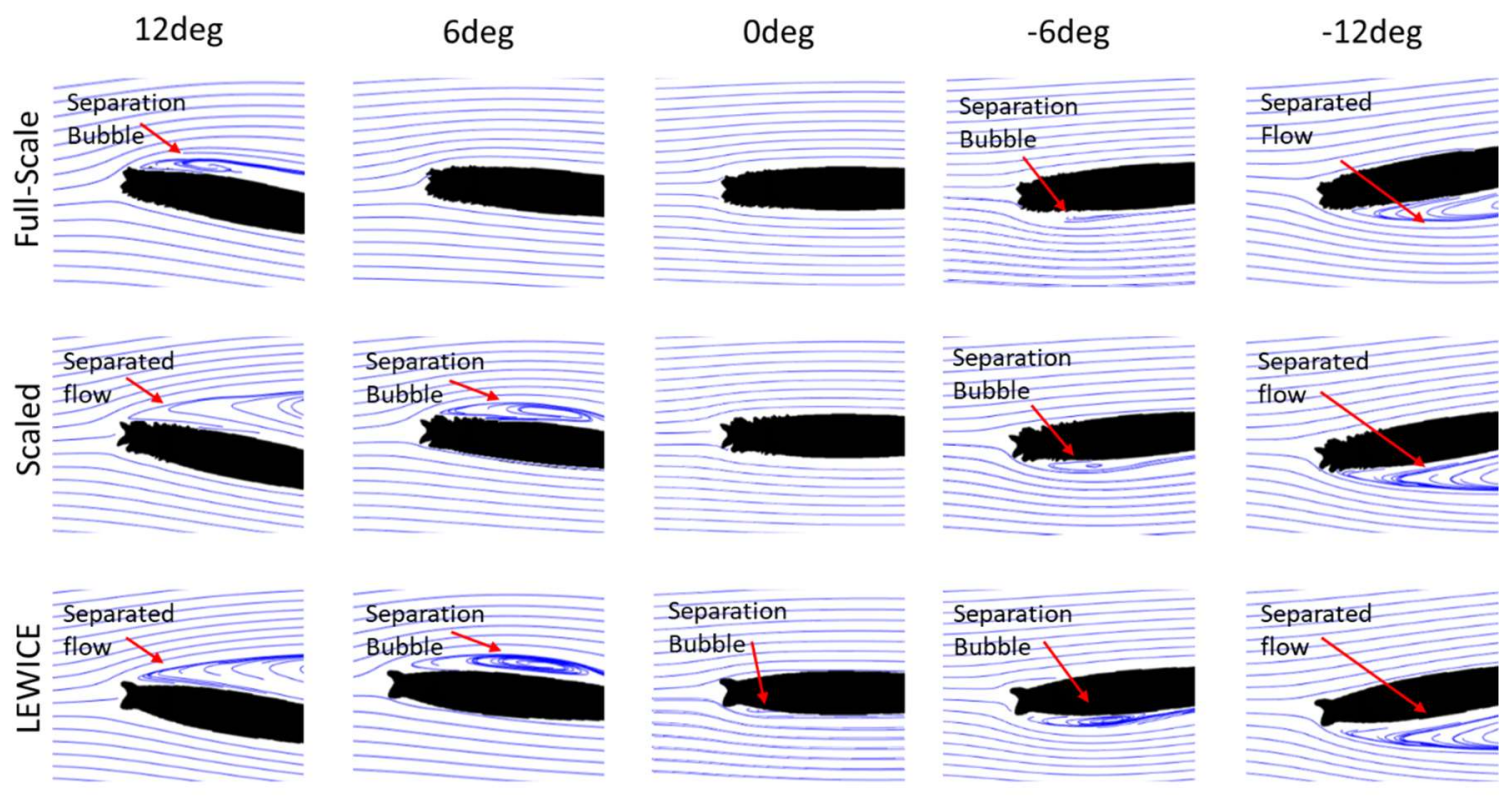

Figure 43: On-body streamlines for $n_{0}=0.5$ ice shapes

At an angle of attack of $12^{\circ}$, the flow is fully separated for the LEWICE and scaled ice shapes, but not the full-scale, as annotated in Figure 43. This trend correlates well with the forcebased results where the scaled and LEWICE ice shapes stalled prior to $12^{\circ}$, indicated by the sudden drop off in the lift coefficient. This same drop off in the lift coefficient was not seen for the fullscale case. A separation bubble on the upper surface of the full-scale can be seen in Figure 43, which could have reattached near the trailing edge preventing flow separation. The near wake flow physics show thicker wake widths with respect to the scaled and LEWICE ice shapes than the fullscale. This is corroborated by the significant increase in the drag coefficient in the scaled and LEWICE ice accretions when compared to the full-scale, as shown in Figure 39. 
At $6^{\circ}$, the scaled and LEWICE ice shapes both have a similar large upper surface separation bubble, as shown in Figure 43, whereas no visible separation is present on the upper surface of the full-scale ice shape. The lack of separation causes the wake width of the full-scale ice shape to be smaller than the other two ice shapes. The resultant decrease in wake momentum deficit in the fullscale ice shape when compared to the other two ice shapes is corroborated by the drag coefficient variation shown in Figure 39.

The LEWICE ice shape is the only ice shape that shows a noticeable separation bubble at $0^{\circ}$ angle of attack, as seen in Figure 43. This results in a wake width thicker than the full-scale and scaled ice shapes. This directly corresponds with the LEWICE ice shape having the greatest coefficient of drag at $0^{\circ}$, as shown in Figure 39.

All three ice shapes show a separation bubble on the lower surface at $-6^{0}$, as annotated in Figure 43. The near wake flow physics show wakes of similar widths and strengths, with the fullscale wake width being slightly thinner than the scaled and LEWICE ice shapes. This is corroborated by the slightly lower drag coefficient of the full-scale ice accretion in Figure 39.

The wake physics of the three ice shapes are again similar at $-12^{\circ}$, with the LEWICE wake being slightly stronger than the other two ice shapes, coinciding with the drag coefficient deviation in Figure 39. On-body, all three ice shapes show flow separation, but the full-scale separation region is significantly shorter than separation caused by the LEWICE and scaled ice shapes.

\subsection{4 $n_{0}=0.5$ Ice Accretions Summary}

In terms of reproducing the streamwise mean velocity component of the full-scale ice shape, the LEWICE and scaled are comparable. Both of the ice shapes show greater regions of separation and thicker and stronger wakes than the full-scale at positive angles of attack. This is due to the upper horn of the LEWICE and scaled ice shapes having a greater horn height than that of the full-scale ice shape. At negative angles of attack, both airfoils produce streamwise mean velocity flow physics similar to the full-scale, with the scaled ice shape being slightly more similar than the LEWICE ice shape. This is corroborated by the drag coefficient curves in Figure 39 and 
is due to the lower horn of all three ice shapes being highly in similar geometry, as shown in Figure 17.

\subsection{Vorticity}

The vorticity of the flow was analyzed to study the angular rotation associated with the flow field. According to Crocco's theorem, movement of vorticity with a given velocity leads to total pressure loss. As such, along with identifying the magnitude of the rotation in the flow field caused by the ice shapes, the vorticity analysis will also indicate the regions of total pressure loss, which ultimately leads to an increase in drag. As discussed, the aerodynamics of horn iced airfoils are dominated by the presence of a separation bubble. This separation bubble causes a shear layer to form between it and the higher velocity free stream flow. Due to the pressure of dominant unidirectional velocity gradients, the shear layer shows higher magnitude of vorticity and leads to vortex shedding. A comparison of the vorticity present in the flow fields of each of the ice shapes is presented in the same manner as the normalized mean U-velocity. Each set of iced shapes will be discussed separately. The vorticity is normalized by multiplying by chord and dividing by the freestream.

\subsection{1 $n_{0}=0.3$ Ice Accretions Results}

Figure 44 includes the vorticity contours for the ice shapes collected at $n_{0}=0.3$. It is important to note that the contour range differs between the on-body and free shear layer contours. The contour range for the on-body and near wake contours are provided on the left and right-hand sides of the figure, respectively. Ideally, the vorticity in the upper and lower surface boundary layer should have vorticity of opposite signs as seen in the near wake. However, in the contour images below, both upper and lower surface vorticity have a negative sign. This is a result of performing PIV on the upper and lower surface separately and post processing the upper and lower surfaces separately. Since the orientation of the camera with respect to the wing remained constant in both the upper and lower surface PIV measurements, the vorticity on both surfaces show the same sign. In the present context, the vorticity magnitude is more significant than its sign. 

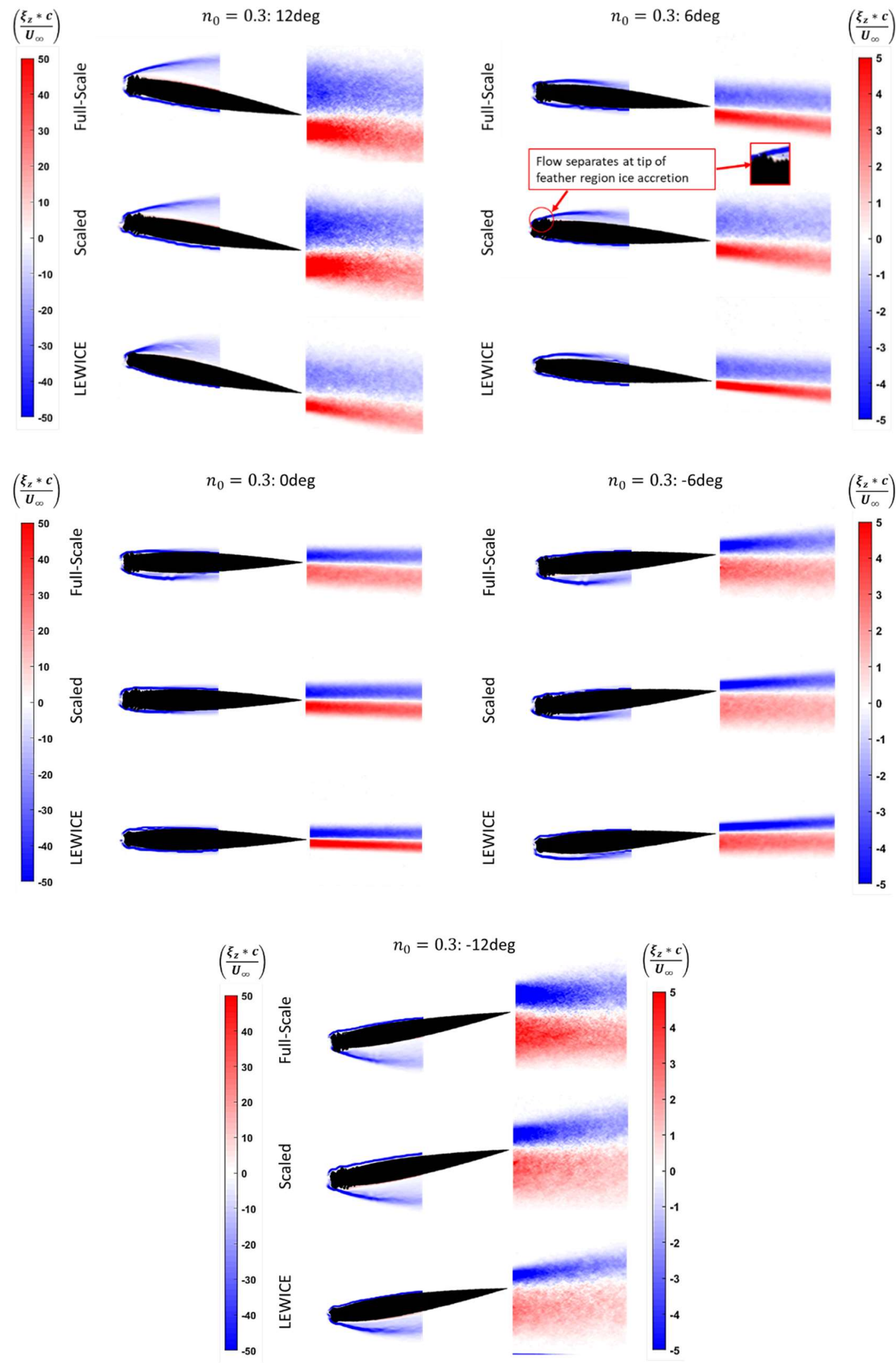

Figure 44: Normalized vorticity contours for $n_{0}=0.3$ ice shapes 
As shown by the vorticity contours, the full-scale and scaled iced shapes produce similar vorticity magnitude and distribution at an angle of attack of $12^{\circ}$. The fully separated flow on the upper surface of the full-scale and scaled ice shapes generates large-scale vorticity with similar magnitude and distribution indicating similar angular rotation and total pressure loss. A different magnitude and distribution of vorticity is seen in LEWICE ice shape on both the upper surface and in the near wake when compared to the other two cases. The upper surface vorticity is more dispersed when compared to the full scale and the scaled ice shapes resulting in lower magnitude vorticity in the near wake.

The similarities in the flow field between the full-scale and the scaled ice shape breaks apart around $6^{\circ}$ angle of attack, as observed in the normalized mean $U$ velocity. A higher vorticity magnitude and width is seen on the upper surface of the scaled ice shape when compared to the other cases. While the strength of the vorticity in the wake is weaker than the wake of the full-scale ice shape and the LEWICE ice shape, the wake width is certainly greater in the scaled ice shape. The difference between the scaled and full-scale ice shapes was mentioned to possibly be due to the large significantly aft feather region ice formation of the scale ice shape. The vorticity contours clearly support this hypothesis, as a second separation point is clearly located at the tip of this feather region formation, as annotated in Figure 44, and previously shown by the mean U-velocity component contours. Overall, the LEWICE ice shape produces similar vorticity in the flow as the full-scale at $6^{\circ}$ angle of attack.

At $0^{\circ}$ angle of attack, the scaled ice shape represents the full-scale better than the LEWICE ice shape as indicated by similar wake thickness and on-body and near wake vorticity distribution. The vorticity magnitude in the wake, however, is lower in the full-scale ice shape when compared to the scaled ice shape. The lower surface separation bubble of the full-scale ice shape might be the cause for the larger wake and less concentrated vorticity when compared to the other two cases. As mentioned, the separation bubble at the lower surface is caused by the taller lower horn on the fullscale ice shape. The presence of the separation bubble continues throughout the negative angles of 
attack until stall is reached by both the full-scale and scaled ice accretions. The LEWICE ice shape has a thinner wake with higher magnitude concentrated vorticity compared to the other ice shapes.

The $-6^{\circ}$ angle of attack case offers similar trends as seen in the $0^{\circ}$ angle of attack between the three cases. The LEWICE ice shape, once again, creates a smaller wake with stronger vorticity throughout the wake, while the full-scale and scaled ice shape show very similar trends.

At $-12^{\circ}$ angle of attack, the region of rotational flow is similar in size across all three ice shapes, but the vorticity generated by the full-scale is the strongest, showing a greater pressure loss. The vorticity in the wake of the scaled ice shape is more similar in strength to the LEWICE ice shape, even though the flow is fully separated.

\subsection{2 $n_{0}=0.3$ Ice Accretions Summary}

Based on the vorticity plots, it can be concluded that the scaled ice shape was decently representative of the full-scale ice shape, whereas the LEWICE ice shape was not. At the midrange positive angles of attack, the LEWICE ice shape was more characteristic of the full-scale than the scaled. This was found to be due to the large aft feather region accretion of the scaled ice shape when compared to the full-scale. The dispersed vorticity magnitude on the upper surface of the scaled ice shape wing confirmed this suspicion. At an angle of attack of $6^{\circ}$, a second flow separation point can be seen at the tip of the upper feather region ice accretion of the scaled ice shape as annotated in Figure 44. The vorticity of the scaled and full-scale ice shapes is comparable from $0^{\circ}$ through $-12^{\circ}$, whereas the LEWICE was only similar at $-12^{\circ}$.

\subsection{3 $n_{0}=0.5$ Ice Accretions Results}

Figure 45 includes the vorticity contours for the ice shapes collected at $n_{0}=0.5$. It is important to note that the contour range differs between the on-body and free shear layer contours. The contour range for the on-body contours is provided on the left-hand side of the figure and the contour range for the free shear layer is provided on the right-hand side of the figure. 

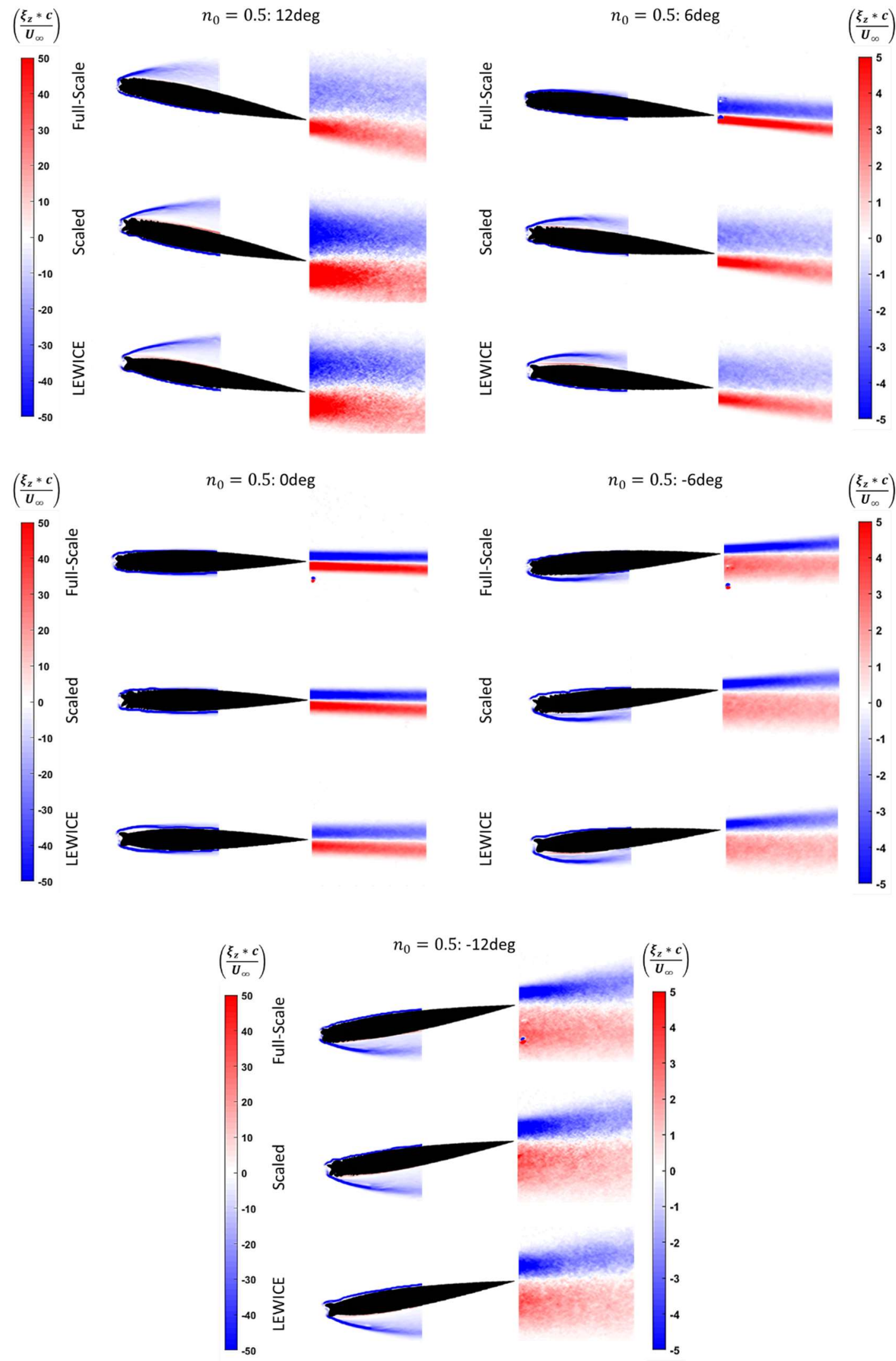

Figure 45: Normalized vorticity contours for $n_{0}=0.5$ ice shapes 
At $12^{\circ}$ angle of attack, the fully-separated flow on the upper surface of the LEWICE and scaled ice shape generates large-scale vorticity with similar magnitude and separation. The vorticity in the wake of the scaled ice shape is slightly higher in magnitude than the LEWICE indicating slightly greater total pressure loss. The separation bubble on the upper surface of the full-scale model produces a different magnitude and distribution of vorticity on both the upper surface and in the near wake when compared to the other two cases. The full-scale upper surface vorticity is more dispersed and results in a lower magnitude vorticity in the near wake.

At $6^{\circ}$ angle of attack, the LEWICE and scaled ice shapes, again, have upper surface and near wake vorticity of similar magnitude and distribution. A higher vorticity magnitude and width is seen on the upper surfaces of the LEWICE and scaled ice shape in comparison to the full-scale ice shape. The greater dispersion leads to lower magnitude vorticity in the wake compared to the full-scale ice shape, where higher vorticity magnitude is observed over a significantly smaller area. The difference between the LEWICE and scaled ice shapes from the full-scale can be attributed to the taller upper horn structures generating larger separation bubbles, as shown in Figure 43. The larger separation bubbles lead to a larger upper surface shear layer.

The scaled and full-scale ice shapes show similar behavior at $0^{\circ}$, both showing less flow separation than the LEWICE ice shape and as a result exhibit a thinner wake with high vorticity magnitude. The LEWICE ice shape produces a larger wake in which the vorticity is more dispersed. At $-6^{0}$, all three ice shapes produce similar amounts of rotational flow. The full-scale has a smaller region of vorticity over its lower surface than the scaled and LEWICE ice shapes, which leads to a slightly thinner wake. The magnitude of the wake vorticity is highly similar across all three ice shapes. However, the wake vorticity magnitude differs in the region corresponding to the upper surface. The feather regions of the full-scale and scaled ice shapes cause higher magnitude vorticity on the lower surface, whereas the smoothness of the LEWICE ice shape in the same region results in lower magnitude vorticity. 
At $-12^{\circ}$ angle of attack, the region of rotational flow is similar in size across all three ice shapes, but the vorticity generated by the full-scale is slightly stronger in the wake with respect to the vorticity generated by the upper surface. The similarity in the vorticity between the three ice shapes shows similar total pressure loss and is expected since the lower horn shape of the three ice shapes are all similar.

\subsection{4 $n_{0}=0.5$ Ice Accretions Summary}

The vorticity analysis of the $n_{0}=0.5$ ice accretions expresses the necessity of preserving the horn geometry to maintain dynamic similitude with full-scale ice accretion. Both the LEWICE and scaled ice shapes varied from the full-scale at the positive angles of attack. The upper horn geometry of the LEWICE and scaled ice shape vary significantly from the full-scale upper horn. This variation in the upper horn is the reason for the deviation from the full-scale at positive angles of attack. At $0^{0}$ angle of attack, the scaled ice shape is decently characteristic of the full-scale, whereas the LEWICE ice shape is not. This can be attributed to the lack of feather region icing on the LEWICE ice shape resulting in greater separation. The lack of a feather region results in a more abrupt geometry change generating greater separation. The LEWICE and scaled ice shapes both produce similar vorticity magnitude and dispersion at the negative angles of attack. This similarity reiterates the importance of preserving the horn ice structure. The lower horns of all three ice shapes are highly similar in geometry and results in vorticity generation as the full-scale at positive angles of attack.

\subsection{Reynolds Stress}

The flow in the upper and lower surface of wings with ice accretions as well as the near wake is inherently turbulent in nature. As such, it is prudent to analyze both the mean and fluctuating component of the flow field. Both the mean velocity component and vorticity represents the behavior of the mean flow properties and doesn't provide insight into the associated turbulence present in the flow. In order to compare the turbulent flow properties, a comparison of the Reynolds

shear stress of the ice shape flow fields is presented in the same style as the mean U-velocity and 
vorticity. The Reynolds shear stress represents the momentum transport due to turbulence generated by the ice accretions. The magnitude of the Reynolds shear stress represents the correlation between the streamwise fluctuating velocity component $\left(u^{\prime}\right)$ and the wall normal fluctuating velocity component $\left(v^{\prime}\right)$. A higher magnitude Reynolds stress represents a higher correlation between these two quantities. The sign of the Reynolds stress represents the direction of the correlation. Positive Reynolds stress indicates both fluctuating velocities have positive values, whereas the negative Reynolds stress indicates either one of the fluctuating velocities have a negative sign. Similar to vorticity, in the present context, the magnitude of the Reynolds stress is more significant than its direction. A stronger Reynolds shear stress magnitude represents higher momentum transport due to turbulence and indicates a correlation between the streamwise $\left(u^{\prime}\right)$ and wall-normal $\left(v^{\prime}\right)$ fluctuating velocity component. Since the Reynolds stress represents the momentum transport due to turbulence, any minor deviations in the flow field due to different ice accretions are amplified. The Reynolds stress discussed in this section is normalized by the square of freestream velocity.

\subsection{1 $n_{0}=0.3$ Ice Accretions Results}

Figure 46 includes the Reynolds stress contours for the ice accretions collected at $n_{0}=$ 0.3. It is important to note that the contour range differs between the on-body and free shear layer contours. The contour range for the on-body contours is provided on the left-hand side of Figure 46 and the contour range for the free shear layer contour is provided on the right-hand side of Figure 46. The differences seen between the three cases in the normalized U velocity and the normalized vorticity is amplified in the Reynolds stress contours. 

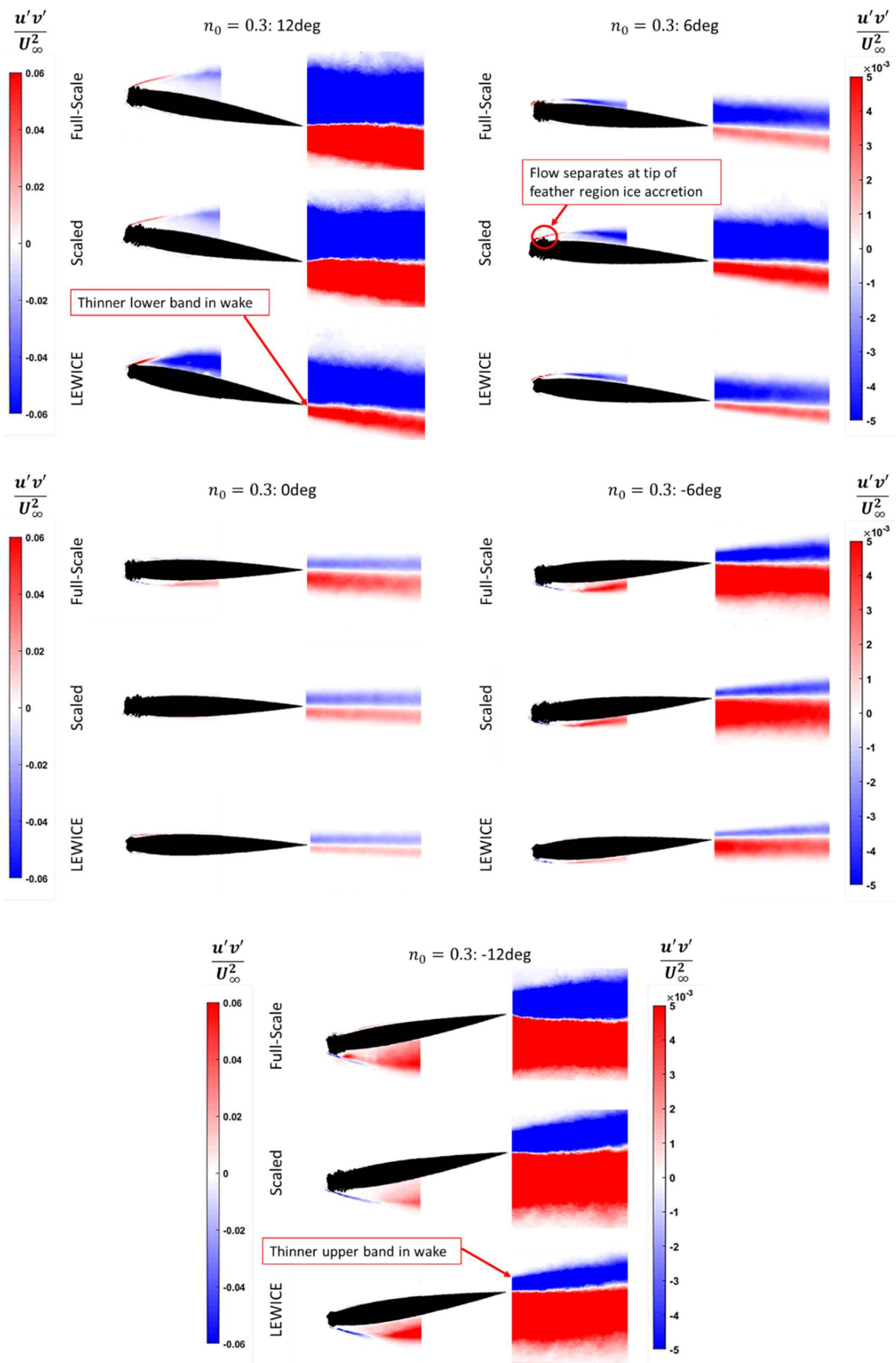

Figure 46: Reynolds stress contours for $n_{0}=0.3$ ice shapes 
Strong negative Reynolds stress is observed in the upper surface of the LEWICE shape when compared to the full-scale and the scaled ice shapes at $12^{\circ}$. It is interesting to note that the higher magnitude of the Reynolds stress is seen closer to the leading edge in the LEWICE shape when compared to the other two cases. A strong Reynolds stress could also indicate the presence of smaller coherent length scales when compared to the other two ice shapes, which will be confirmed by performing modal analysis and is discussed in the next chapter. The Reynolds stress bands in the near wake also show similar trends between the full-scale and the scaled wing model when compared to the LEWICE. It should be noted that the wake Reynolds stress corresponding to the lower surface of the wing (red region) has similar thickness between the full-scale and the scaled ice shape, whereas the thickness is significantly thinner in the LEWICE shape. This shows less momentum transport due to turbulence from the flow exposed surface of the LEWICE ice shape compared to the other two ice shapes.

As seen in the U-velocity and vorticity contours, similar deviations are observed in the Reynolds stress between the full-scale and scale ice shapes at $6^{\circ}$ angle of attack which also coincides with the drag coefficient variation. As observed in the vorticity contours, a clear separation point at tip of large feather accretion is seen on the scaled ice shape. At $6^{0}$ angle of attack, the LEWICE ice shape is characteristic of the full-scale, having similar turbulence transition points in the shear layer generated by the upper surface ice shape. The Reynolds stress in the wake is highly similar between the LEWICE and full-scale, whereas the Reynolds stress in the wake of the scaled ice shape is significantly greater.

The $0^{\circ}$ angle of attack vorticity contours showed that the LEWICE shape is more streamlined than the full-scale and the scaled models. The presence of a larger lower separation bubble in the lower surface of the full-scale model is not successfully replicated either by the scaled or the LEWICE ice shapes. This led to the differences seen in the near wake where the full-scale ice shape Reynolds stress corresponding to the lower surface (red band) has a thicker width when 
compared to the lower surface Reynolds stress from the other two cases. The magnitude of the Reynolds stress from the LEWICE shape is much lower than that of the full scale.

The near wake Reynolds stress thickness and magnitude associated with the lower surface between the full-scale and scaled ice shape compare extremely well at $6^{\circ}$ angle of attack with the LEWICE ice shape failing to match the trends. The LEWICE ice shape produces significantly less turbulence in the wake and on-body than the other two ice shapes.

The near wake Reynolds stress thickness and magnitude associated with the lower surface compares well for all three ice shapes at $-12^{\circ}$. The Reynolds stress band in the near wake due to the upper surface is noticeably thinner for the LEWICE ice shape compared to the other two ice shapes, showing less momentum transport due to turbulence from the flow exposed surface of the wing. It is interesting to note the higher magnitude Reynolds stress closer to the lower surface leading edge for both the LEWICE and full-scale compared to the scaled ice shape at $-12^{\circ}$ angle of attack. The stronger Reynolds stress could indicate the presence of smaller coherent length scales for the fullscale and LEWICE ice shapes compared to the scaled ice shape. This will be confirmed by the POD modal analysis results.

\subsection{2 $n_{0}=0.5$ Ice Accretions Results}

Figure 47 includes the Reynolds stress contours for the $n_{0}=0.5$ ice shapes. It is important to note that the contour range differs between the on-body and free shear layer contours. The contour range for the on-body contours is provided on the left-hand side of Figure 47 and the contour range for the free shear layer contour is provided on the right-hand side of Figure 47. As was the case for the first set of ice shapes, the Reynolds stress contours showed similar trends as found in the vorticity contours. 

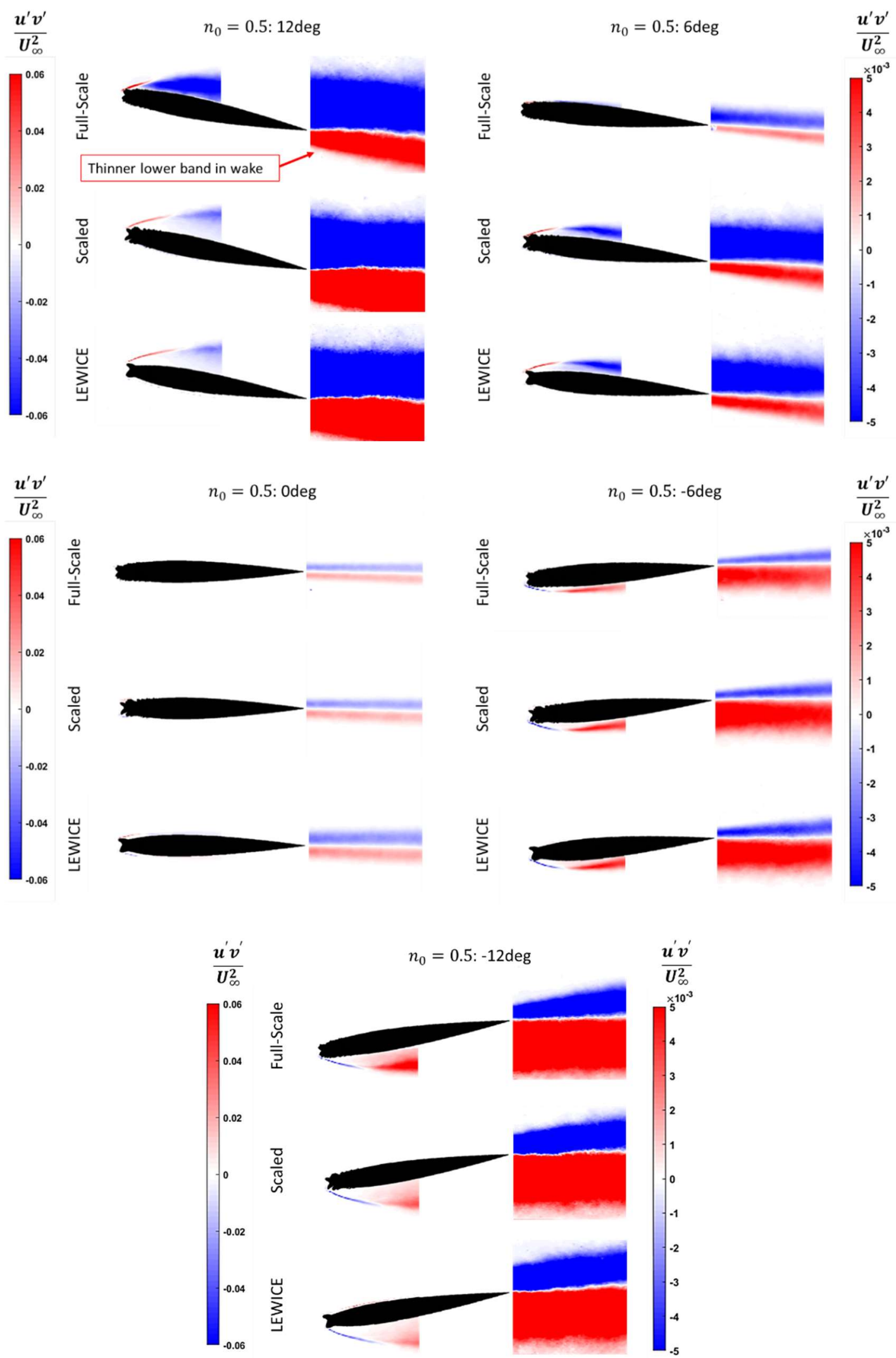

Figure 47: Reynolds stress contours for $\boldsymbol{n}_{0}=0.5$ ice shapes 
A strong negative Reynolds stress is observed on the upper surface of the full-scale ice shape when compared to the other two ice shapes at $12^{\circ}$. This indicates that the flow field on the upper surface of the full-scale ice shape is more turbulent when compared to the other two cases. All three ice shapes have Reynolds stress bands corresponding to the upper surface (blue band) of similar thickness, but the Reynolds stress band corresponding to the lower surface (red band) of the full-scale is significantly thinner than the other two ice shapes as annotated in Figure 47.

The vorticity plot showed similarity between the LEWICE and full-scale ice shapes at $6^{0}$, however this is not the case with respect to the Reynolds stress. The scaled and LEWICE ice shapes have a strong negative Reynolds stress on the upper surface, whereas almost no Reynolds stress is present on the upper surface of the full-scale ice shape. This corresponds with the normalized streamwise velocity contours. The near wake Reynolds stress bands of the full-scale ice shape are significantly thinner than those of the other two ice shapes. The near wake Reynolds stress magnitude is also slightly lower than the scaled and LEWICE ice shapes, showing less momentum transport due to turbulence with respect to the full-scale ice shape.

This trend continues at $0^{\circ}$, where the near wake Reynolds stress bands of the full-scale are again the thinnest. The scaled is more similar to the full-scale than the LEWICE, which has significantly thicker bands in the wake. This corresponds with the thicker wake of the LEWICE ice shape shown by both the normalized velocity and vorticity contours. All three ice shapes have minimal Reynolds stress on either the upper or lower surface.

The Reynolds stress contours at $-6^{0}$ continue to show higher Reynolds stress in the LEWICE and scale ice when compared to the full-scale ice shape. Strong positive Reynolds stress is seen on the lower surfaces and near wake of the LEWICE and scaled ice shapes. The Reynolds stress on the lower surface and in the near wake of the full-scale ice shape is lower in magnitude and thinner than those of the LEWICE and scaled ice shapes. 
A strong Reynolds stress band corresponding to the lower surface can be seen throughout the near wake contours of all three ice shapes at $-12^{\circ}$ angle of attack. The Reynolds stress corresponding to the upper surface of the full-scale ice shape is thinner than the other two cases.

\subsection{Summary of Chapter V}

The flow field analysis mentioned in this chapter had strong correlations with the lift and drag coefficient results discussed in Chapter 4 . With respect to the $n_{0}=0.3$ ice shapes, throughout the flow field analysis, the scaled ice shape was characteristic of the full-scale ice shape only deviating at the midrange positive angles of attack. As shown previously and again by the Reynolds stress contours, the deviation at the midrange positive between the scaled and full-scale ice shapes is due to the large, aft positioned feather region ice accretion on the upper surface of the scaled ice shape. This was expressed by a second separation point at the tip of the feather region ice accretion. The scaled ice shape was also able to account for the momentum transport due to turbulence well in comparison to the full-scale. The $n_{0}=0.3$ LEWICE ice shape, on the other hand, consistently differed from the full-scale ice shape. This was highlighted by the Reynolds stress analysis, where the LEWICE ice shape continually underpredicts the momentum transfer due to turbulence.

As for the $n_{0}=0.5$ ice shapes, the scaled ice shape inconsistently reproduced the flow field dynamics of the full-scale ice shape. At positive angles of attack, the scaled ice shape differed from the full-scale consistently having a larger wake than the full-scale ice shape at this region of the angle of attack range. The on-body separation also varied at positive angles of attack. The deviation is mostly attributed to the taller horn of the scaled ice shape. The large upper surface secondary horn-like structure of the scaled ice shape could also be partially responsible for the flow field differences, but no clear second separation point was seen. At $0^{\circ}$ and the negative angles of attack, the scaled produced a flow field characteristic of the full-scale. The LEWICE ice shape showed the same inconsistency as the scaled ice shape for the $n_{0}=0.5$ ice shapes. This can be attributed to the upper horn of the LEWICE ice shape being taller than the upper horn of the fullscale and having a greater icing limit, whereas the lower horns were very similar in geometry. 
The ice shape scaling method was able to produce an ice shape characteristic of the fullscale ice shape for the $n_{0}=0.3$ ice shapes, but not for the $n_{0}=0.5$ ice shapes. This again suggests a possible stagnation freezing fraction/temperature constraint of ice shape scaling method recommended by Anderson and Tsao [2] under SLD conditions. The LEWICE software was unable to predict an ice shape that was consistently characteristic of the full-scale for both the $n_{0}=0.3$ and $n_{0}=0.5$ cases. This coincides well with the results of the lift and drag coefficient results that also showed the LEWICE predicted ice shape to be uncharacteristic of the full-scale ice shape. 


\section{CHAPTER VI}

\section{POD FLOW FIELD ANALYSIS}

Modal decomposition of the flow field in the boundary layer and the near wake was performed using Proper Orthogonal Decomposition (POD). The flow fields generated by the different ice shapes were compared based on the energy present in each mode and the corresponding coherent structures. The first mode in the POD analysis is characteristic of the mean flow. Since the mean flow was discussed previously and the coherent structures are of interest, the second and third modes for both the $\mathrm{U}$ and $\mathrm{V}$ velocity fluctuations are presented for each ice shape at $-12^{\circ},-6^{0}, 0^{\circ}, 6^{0}$, and $12^{\circ}$. Prior to discussion of the ice shapes, it is important to show the ability of POD to reproduce the flow field using the different modes, shown in Figure 48.
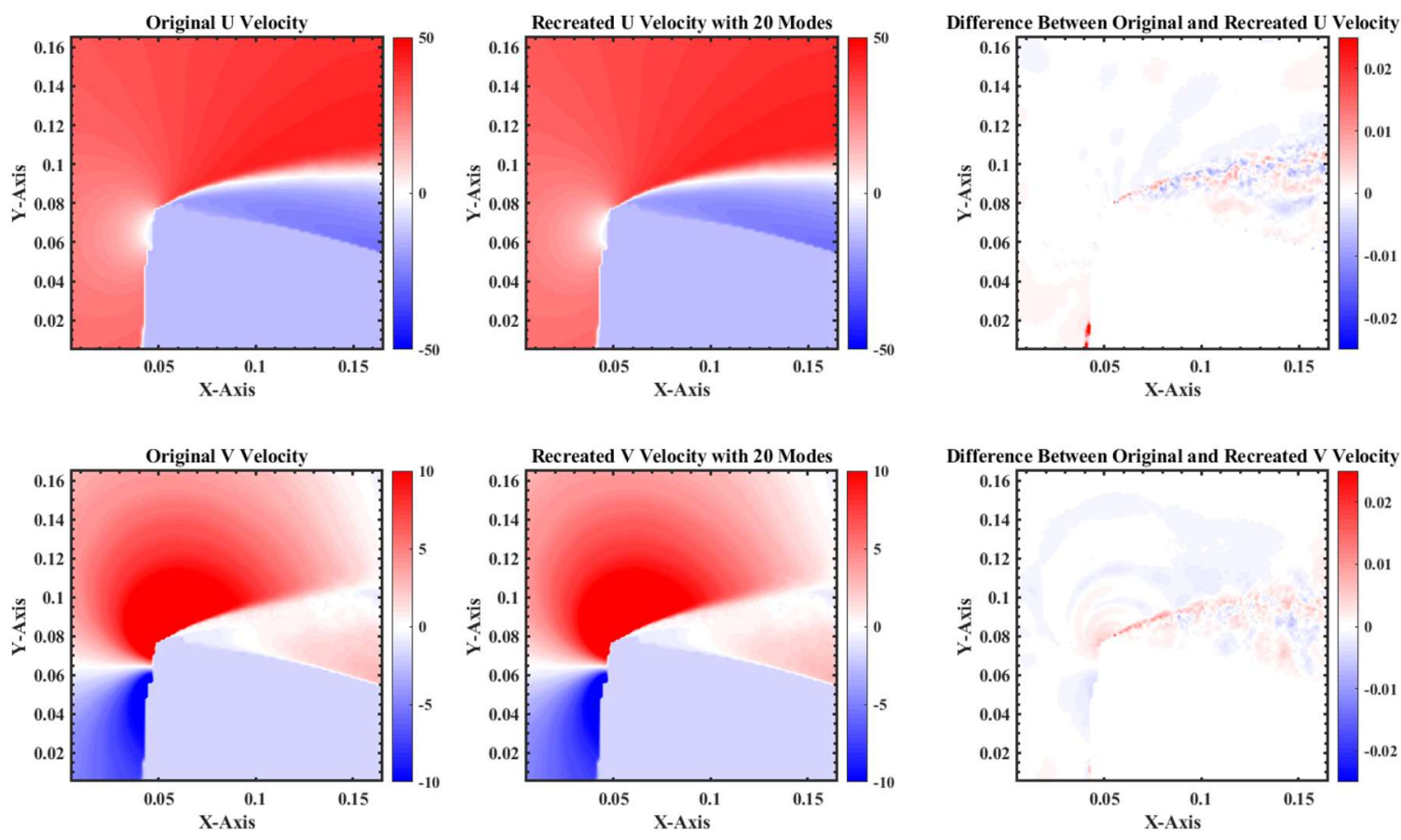

Figure 48: Flow field reconstruction using POD modes

The on-body flow field at a positive angle of attack of $12^{\circ}$ is used to express the success of the POD analysis to recreate the flow field, shown in Figure 48, due to being one of the more complicated flow fields. The flow is reconstructed using the first twenty modes and roughly $40 \%$ 
of the flow field energy for each component of velocity. Even with only using the first twenty modes of the POD modal analysis, the flow field is well reconstructed.

\subsection{POD Modal Analysis for $n_{0}=0.3$ Ice Accretions}

\subsubsection{U-velocity Correlations}

The second and third U-velocity and V-velocity modes of the $n_{0}=0.3$ ice shapes are provided in Figures 49 and 50, respectively. The POD comparison of the ice shapes is performed by studying the shedding and the length scale of the coherent structures generated by each ice shape with respect to each fluctuating component of velocity. The contour range for the on-body and free shear layer flow fields is the same and is provided on the left-hand side of Figures 49 and 50.
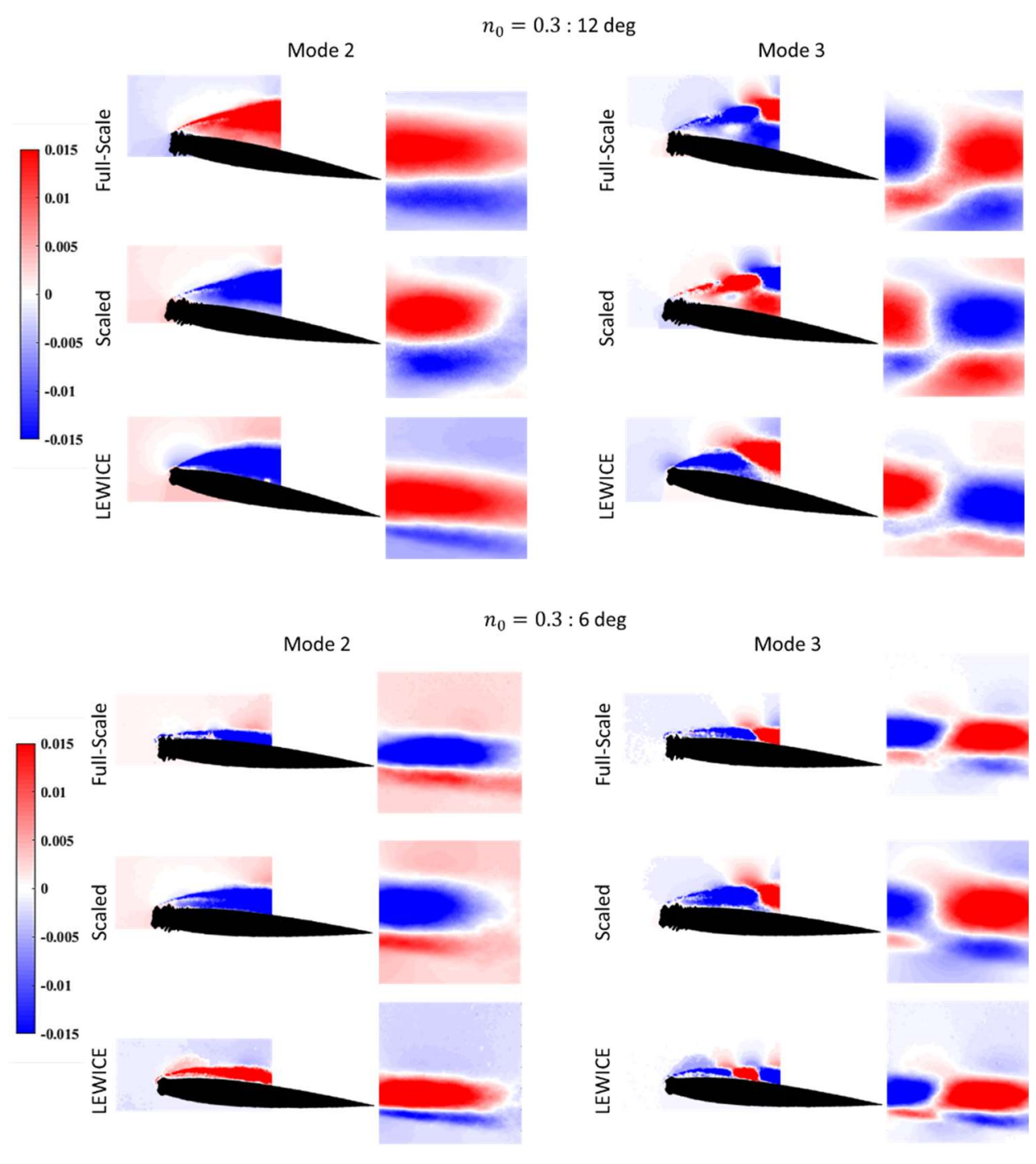

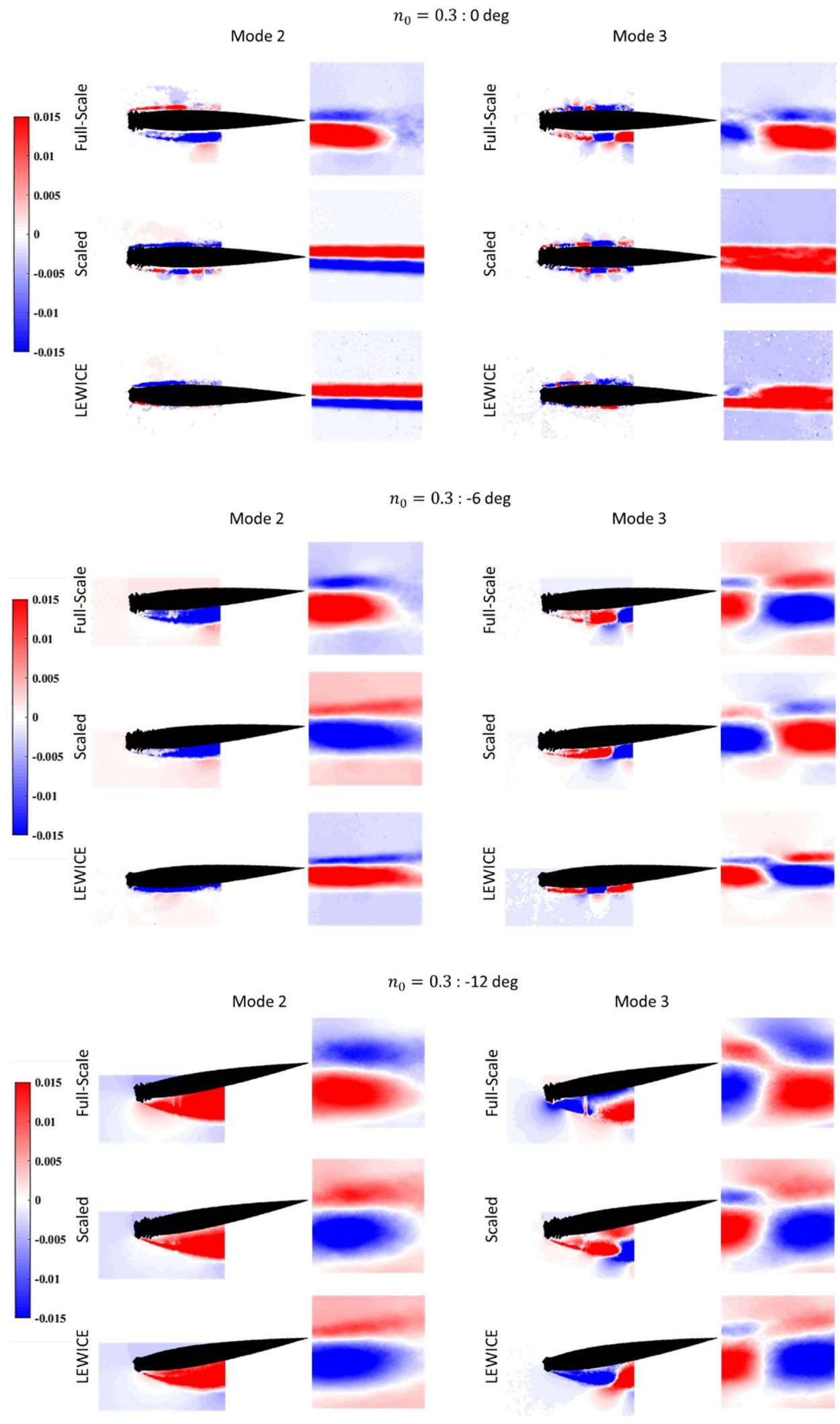

Figure 49: Second and third POD U-velocity correlation modes for $n_{0}=0.3$ ice accretion cases 
Throughout the second mode, no coherent structures are shown in the on-body or wake contours of the $n_{0}=0.3$ ice accretions besides small on-body structures at $0^{\circ}$, with respect to the U-velocity. Large structures may be present in the wake at higher angles of attack, but are too large to be captured within the field of view as possibly suggested by the $12^{\circ}$ scaled wake contour in Figure 49. The U-velocity second mode, highlights the flow separation that occurs due to the ice shapes. The trends shown are the same as were found for the mean U-velocity contours in Chapter V.

The third U-velocity mode shows vortex shedding in the wake throughout the angle of attack sweep for the full-scale ice shape and for both the LEWICE and scaled ice shapes with the exception of the $0^{\circ}$ case. All of the ice shapes show on-body shedding throughout the angle of attack range in the third mode.

The scaled ice shape produces coherent structures of similar length as the full-scale at negative and positive $12^{\circ}$, at which both iced airfoils have stalled and flow has fully separated. At $6^{0}$, the scaled ice shape produces larger structures than the full-scale due to the large feather region ice accretion. However, at $-6^{0}$ the scaled produces slightly smaller U-velocity coherent structures than the full-scale due to the full-scale having a taller lower horn. This is the same reason the fullscale produces larger on-body shedding at $0^{\circ}$ and is the only ice shape for which coherent structures are present in the wake.

The LEWICE predicted ice shape produces U-velocity correlated coherent structures of shorter length at positive and negative $12^{\circ}$ than the full-scale, since it has not yet reached stall. At $6^{0}$, the third mode structures of the LEWICE ice shape are highly similar in size to those of the fullscale, coinciding with the results of the flow field analysis in Chapter V. This is not the case at $-6^{0}$ or at $0^{\circ}$, where the LEWICE ice shape generated coherent structure length scale is shorter than the full-scale. As stated, the full-scale is the only ice shape to have coherent structures present in the wake with respect to $\mathrm{U}$-velocity at $0^{\circ}$, given the field of view size. 


\subsubsection{V-velocity Correlations}

Figure 50 shows the second and third modes with respect to the V-velocity for the $n_{0}=$ 0.3 of ice shapes. As shown, shedding is present and coherent structures can be seen throughout the wake profiles for all ice shapes. The structures in each case can be directly compared with each other based on their size.
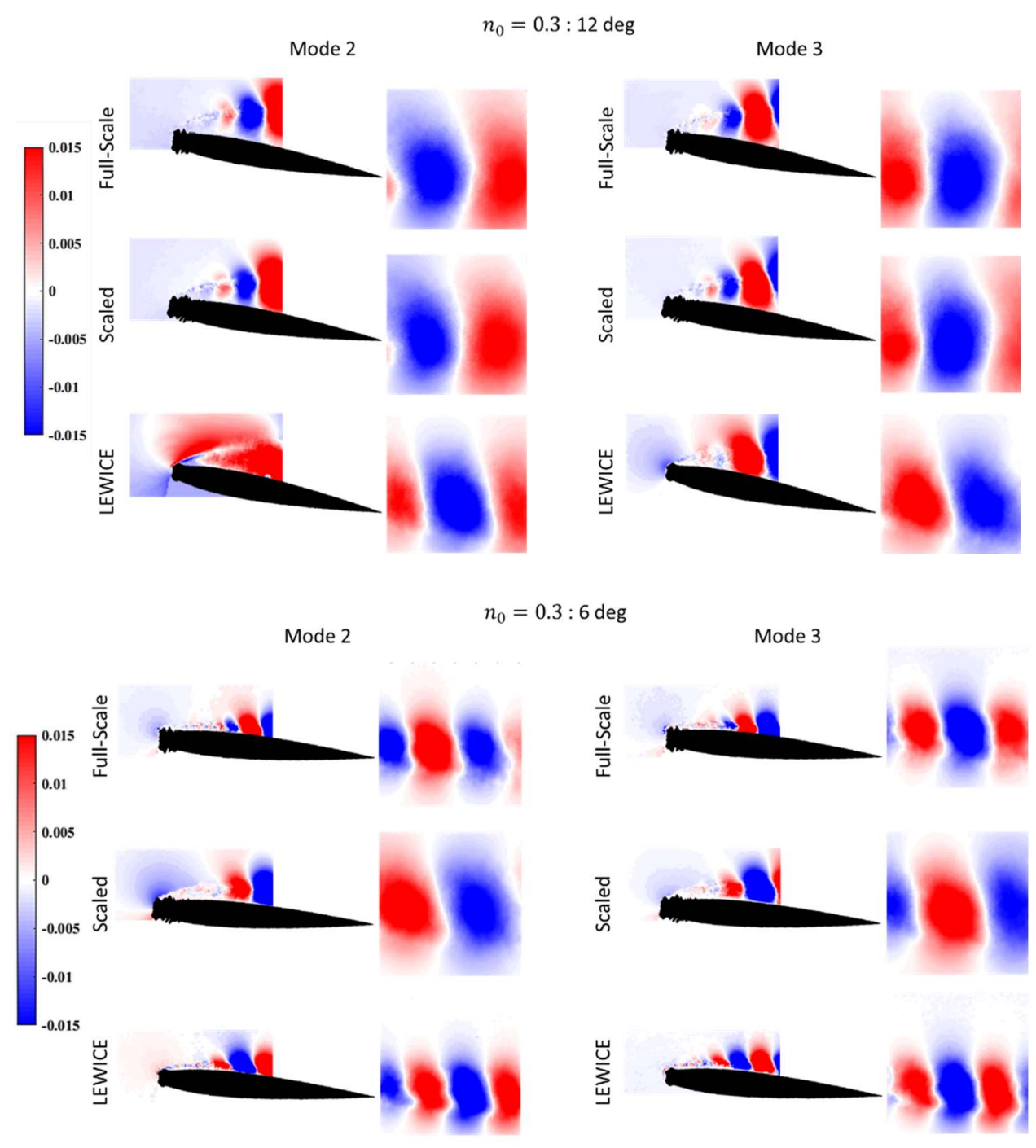

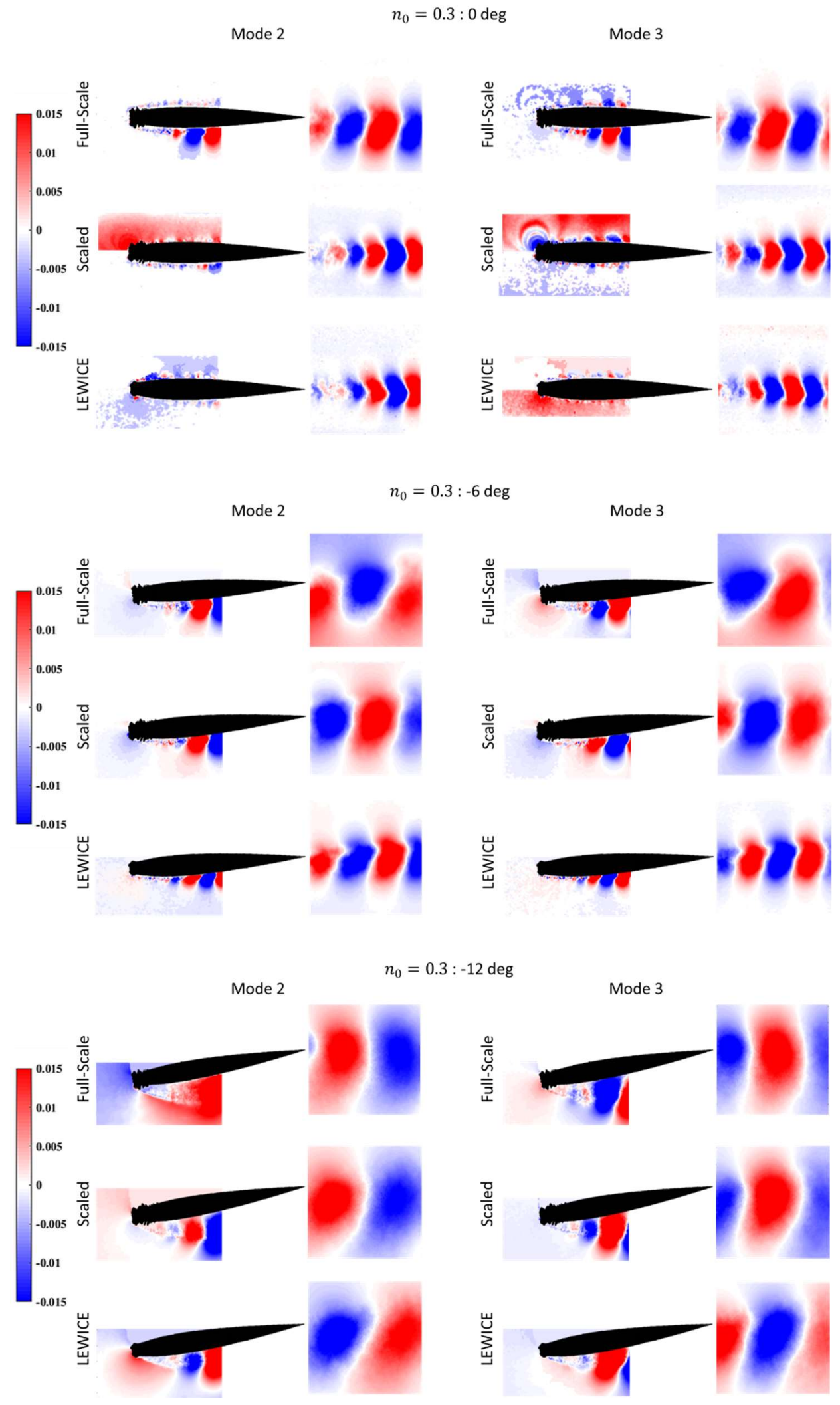

Figure 50: Second and third POD V-velocity correlation modes for $n_{0}=0.3$ ice accretion cases 
The coherent structures in the wake are almost identical between the scaled and full-scale ice shapes at positive and negative $12^{\circ}$ in both the second and third modes. The on-body structures are also very similar at $12^{\circ}$, but vary in the second mode at $-12^{\circ}$. The full-scale on-body contour shows no coherent structures, whereas clearly visible structures are present on the lower surface of the full-scale in the second mode at $-12^{\circ}$. The on-body and wake V-velocity coherent structures are larger for the scaled ice shape in both modes than the full-scale at $6^{0}$. This is due to the comparatively large further aft positioned feather region ice accretion generating greater separation than the full-scale upper surface ice accretion. The effect of the taller lower horn structure of the full-scale can be seen in both modes in the on-body and wake coherent structures at $0^{\circ}$ and $-6^{0}$ angle of attack. At these angles of attack, the coherent structures of the full-scale ice shape, in both modes, are larger than those produced by the scaled ice shape.

When comparing the LEWICE and full-scale ice shapes, the trends found in the U-velocity structures are found in the V-velocity structures. The on-body structures vary in the second mode at $12^{\circ}$ angle of attack, where the LEWICE shows no on-body structures. Though the wake structures are similar in size to the full-scale wake structures at $12^{\circ}$ angle of attack, the LEWICE flow field is dominated by smaller structures, as indicated by the increase in the length scale of the coherent structures between the second and third modes. The full-scale flow field, on the other hand, is dominated by larger structures at $12^{\circ}$ angle of attack. The LEWICE compares well to the full-scale at $6^{\circ}$, though having slightly smaller structures in the second mode than the full-scale. At $0^{\circ}$ and $6^{0}$ angles of attack, the LEWICE produces significantly smaller on-body and wake structures than the full-scale ice shape. The V-velocity structures in the wake are very similar between the LEWICE and full-scale at $-12^{\circ}$, but vary with respect to the on-body in the second mode. The LEWICE ice shape shows on-body structures in the second mode at $-12^{\circ} \mathrm{AOA}$, whereas the fullscale does not. 


\subsubsection{Modal Flow Field Energy Content}

The energy present in the first modes for the first set of ice shapes is presented for both the wake and on-body flow fields in Figure 51 and 52, respectively.
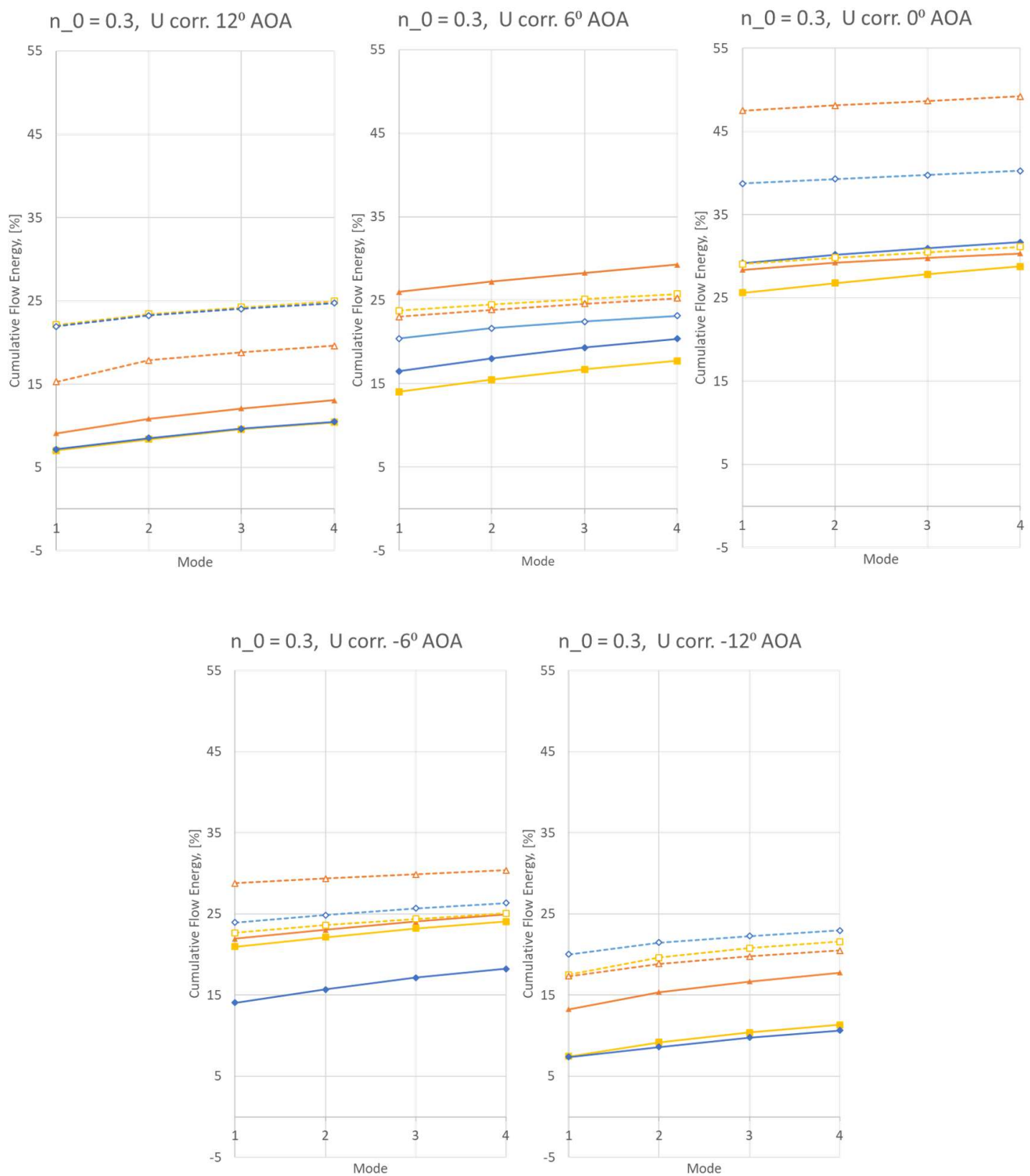

- Full-scale, Free Shear V corr. — Scaled, Free Shear V corr.

- LEWICE, Free Shear V corr.

-- $\square$-- Full-Scale, On-Body V corr.

--৫-- Scaled, On-Body V corr

-- $\triangle--$ LEWICE, On-Body V corr.

Figure 51: Cumulative flow field energy content for first four U-velocity correlation modes for $n_{0}=0.3$ ice accretion cases 


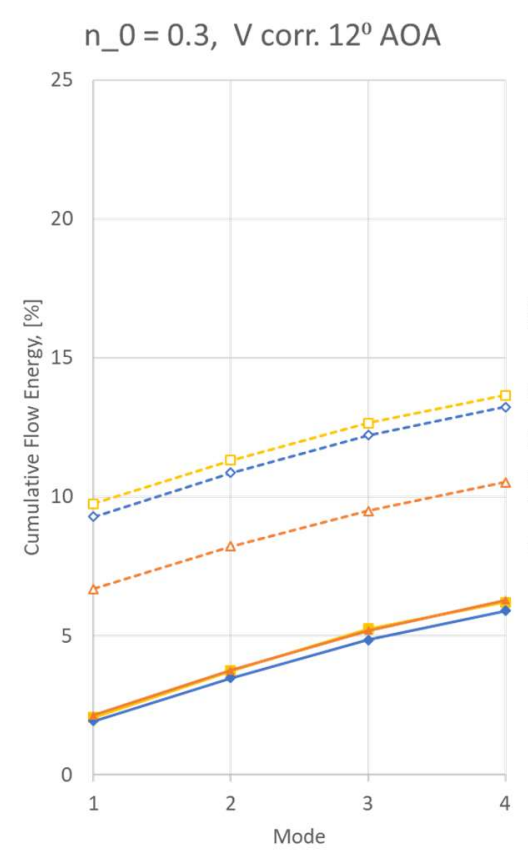

$\mathrm{n} \_0=0.3$, V corr. $6^{0} \mathrm{AOA}$

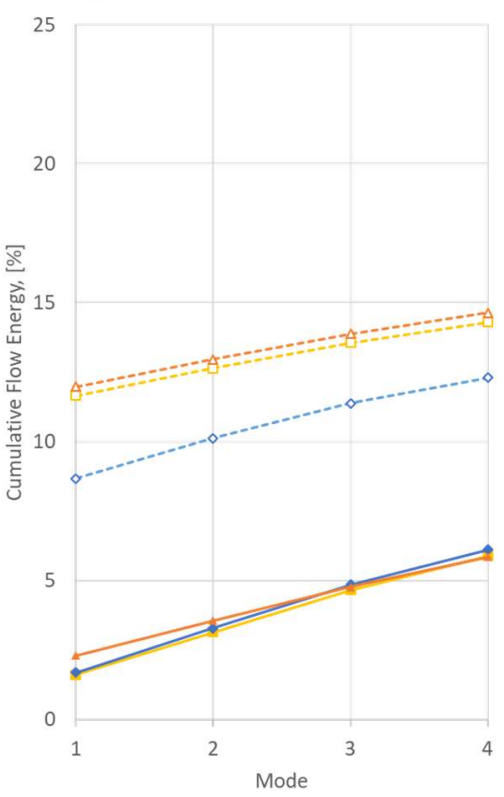

n_0 $=0.3$, v corr. $0^{\circ} \mathrm{AOA}$

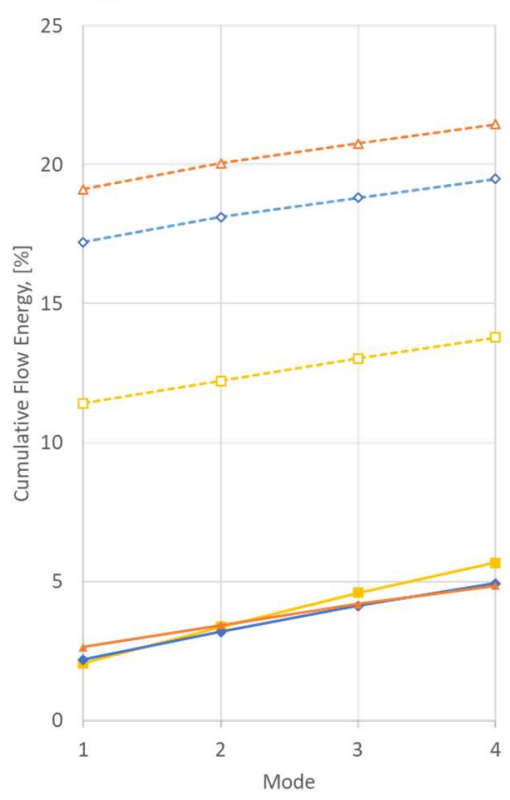

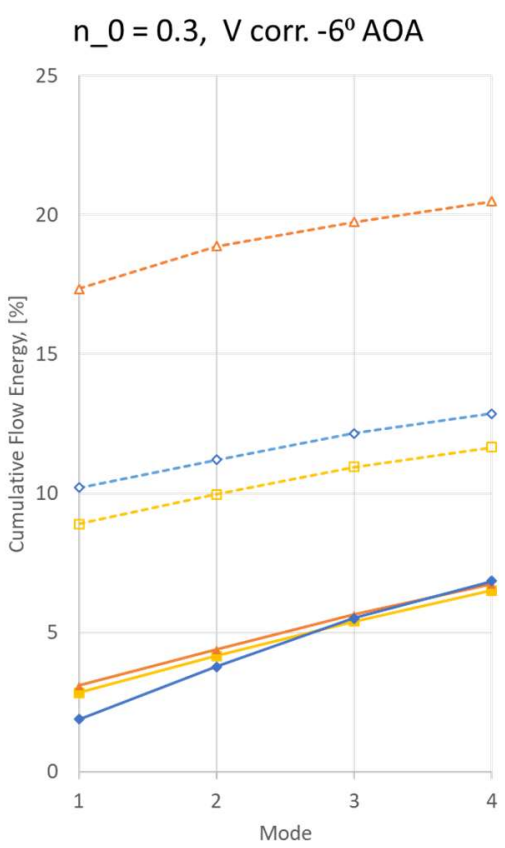

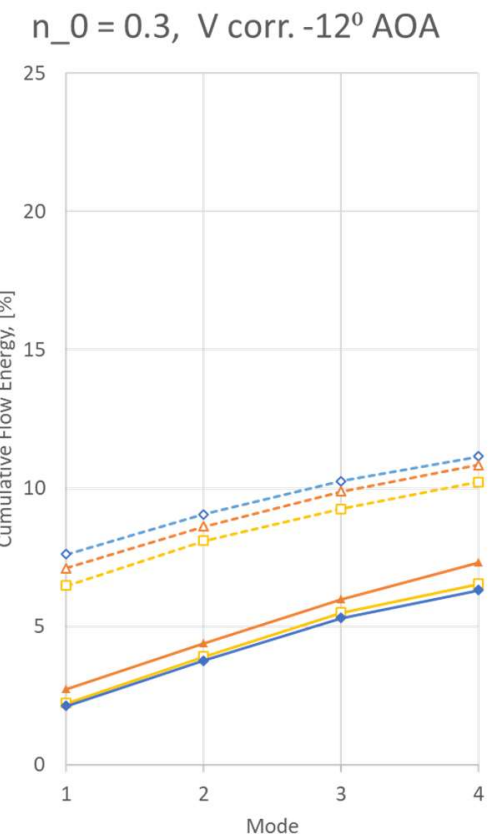

- Full-scale, Free Shear V corr. —- Scaled, Free Shear V corr.

$\longrightarrow$ LEWICE, Free Shear V corr.

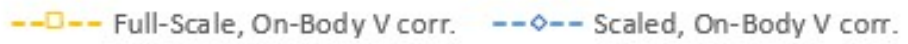
-- $\triangle--$ LEWICE, On-Body V corr.

Figure 52: Cumulative flow field energy content for first four $\mathrm{V}$-velocity correlation modes for $n_{0}=0.3$ ice accretion cases 
There is not much deviation between the flow field energy found in the second through fourth modes between the three ice shapes. The deviations are mostly $<1 \%$ for the second through fourth modes. The primary deviation between the scaled and full-scale ice shapes occurs in both the on-body and wake at $0^{\circ}$ angle of attack, with respect to the energy found in the mean U-velocity mode. This is the angle of attack at which the ice shapes were collected and relates well to the geometric differences of the frontal horn shape, both lower and upper.

It can be seen that the lower horn of the full-scale ice shape is taller than the lower horn on the scaled ice shape. The slight difference in the lower horn causes a large difference in the energy found in the mean flow. The energy present in the U-velocity mean flow differs by $\sim 10 \%$ with respect to the on-body flow and $\sim 4 \%$ with respect to the free shear layer flow. There is also a slight difference in the scaled and full-scale mean U-velocity flow energy at $6^{\circ}$. The on-body energy present in the mean U-velocity flow is $\sim 4 \%$ greater for the full-scale than the scaled ice. This is where the flow field has been greatly influenced by the upper feather region ice accretion. However, the reattachment and pressure recovery of the flow results in a similar mean flow energy content in the free shear layer of less than $2.5 \%$. For the rest of the angles of attack, the scaled and full-scale compare well in different energy modes.

The greatest deviation in the flow energy breakdown between the LEWICE and full-scale ice shapes again occurs in the mean $\mathrm{U}$-velocity energy content at $0^{\circ}$, mainly with respect to the onbody flow field. The energy present in the on-body mean U-velocity flow is $>15 \%$ higher for the LEWICE ice shape than the full-scale ice shape. This major difference is due to the significantly lower overall thickness and volume of the LEWICE ice shape compared to the full-scale. The onbody flow field content also significantly varies at $-6^{0}$, where the difference in the mean flow is $>8 \%$. The rest of the differences are less than $5 \%$ with most being less than $2.5 \%$. Though the energy contents are similar across all three ice shapes, with some exceptions, it is important to remember there is significant difference in the coherent structure size in each of the modes. Therefore, purely looking at the energy breakdown is not sufficient. 
Based on the results of the modal analysis the scaled ice shape was characteristic of the full-scale with the deviation occurring at $6^{0}$ angle of attack coinciding with the trend seen in the aerodynamic coefficients, vorticity, and Reynolds stress contours. The LEWICE ice shape is uncharacteristic across the angle of attack range with the exception of the $6^{0}$ case. This corresponds with the trend seen in the aerodynamic coefficients, vorticity, and Reynolds stress contours, again showing the LEWICE ice shape is uncharacteristic of the full-scale ice shape.

\subsection{POD Modal Analysis for $\boldsymbol{n}_{0}=0.5$ Ice Accretions}

The second and third U-velocity and V-velocity modes of the second set ice shapes collected at $n_{0}=0.5$ are provided in Figures 53 and 54, respectively. The percentage of modal energy for the first four modes is presented in Figure 55 and 56, with the first mode being the mean flow. The contour map for the on-body and free shear layer flow fields is the same and is provided on the left-hand side of Figures 53 and 54.

\subsubsection{U-velocity Correlations}

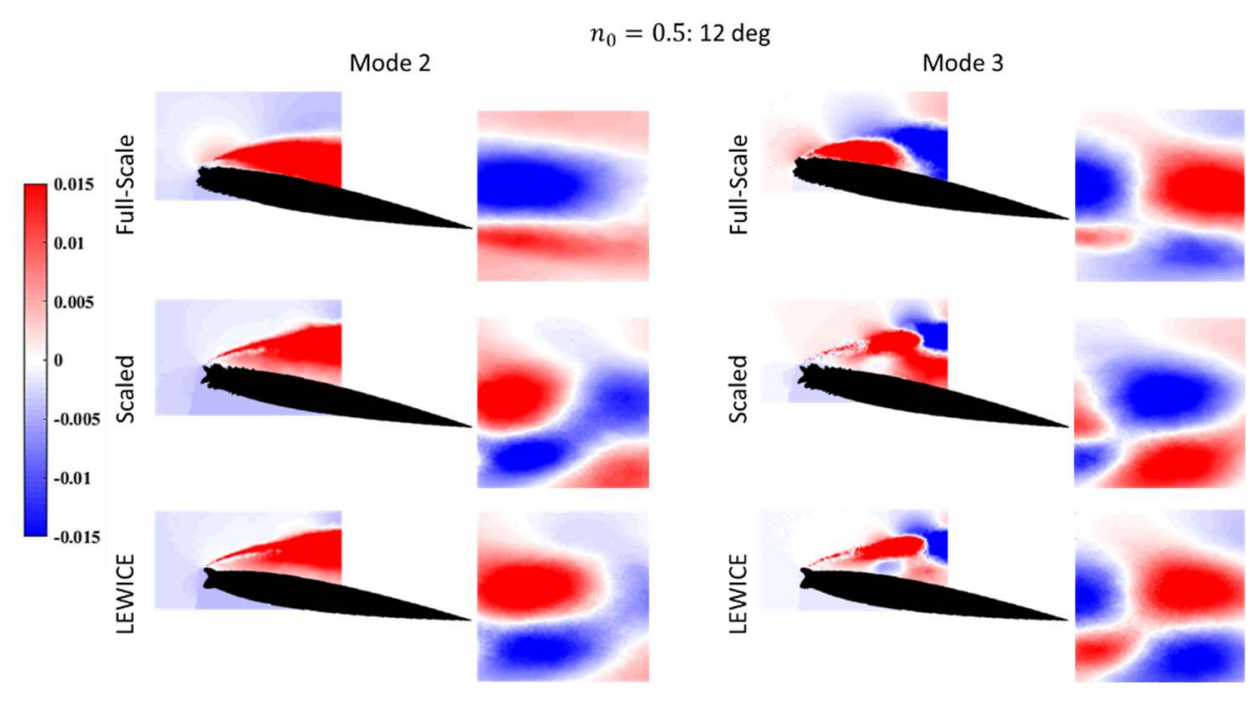



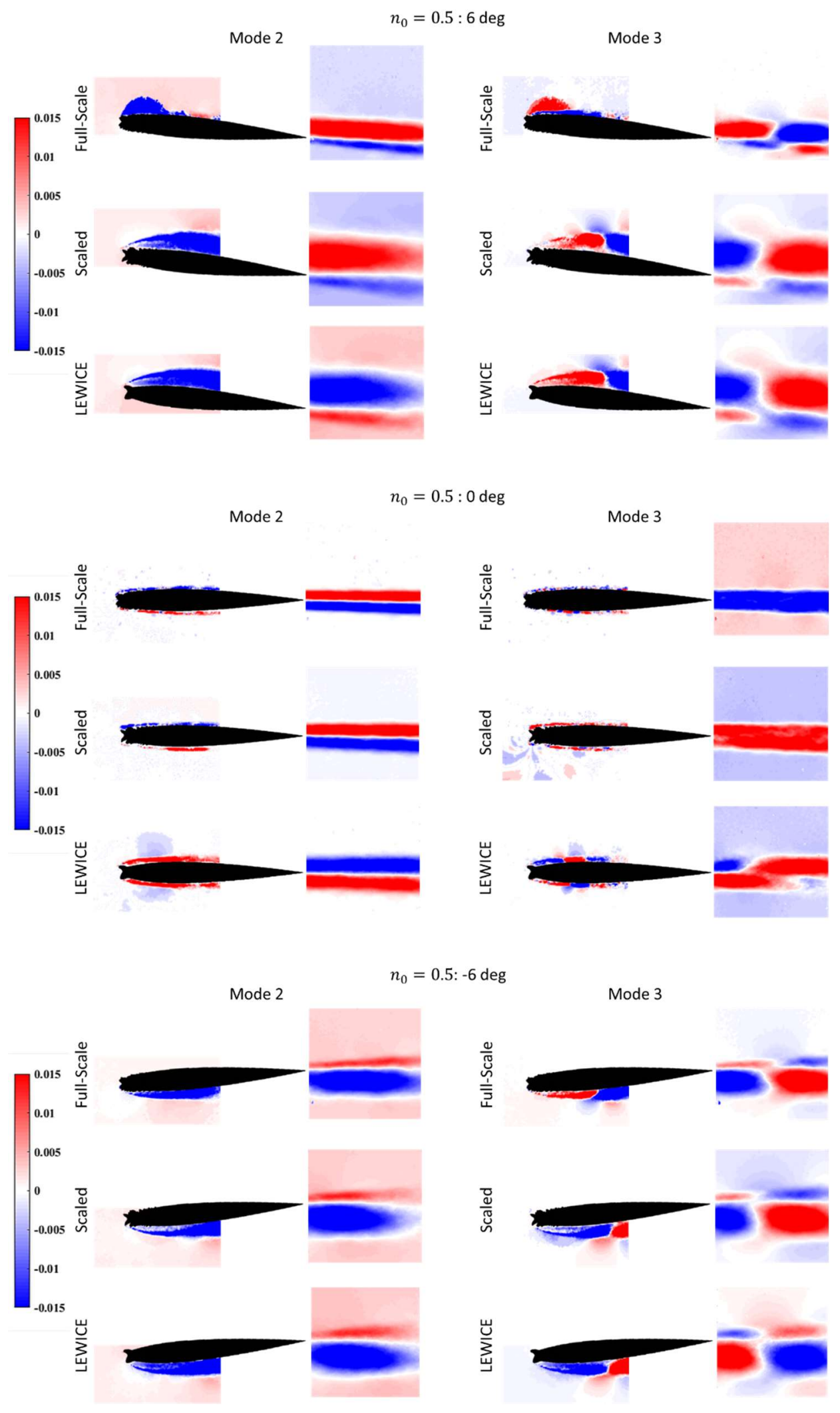


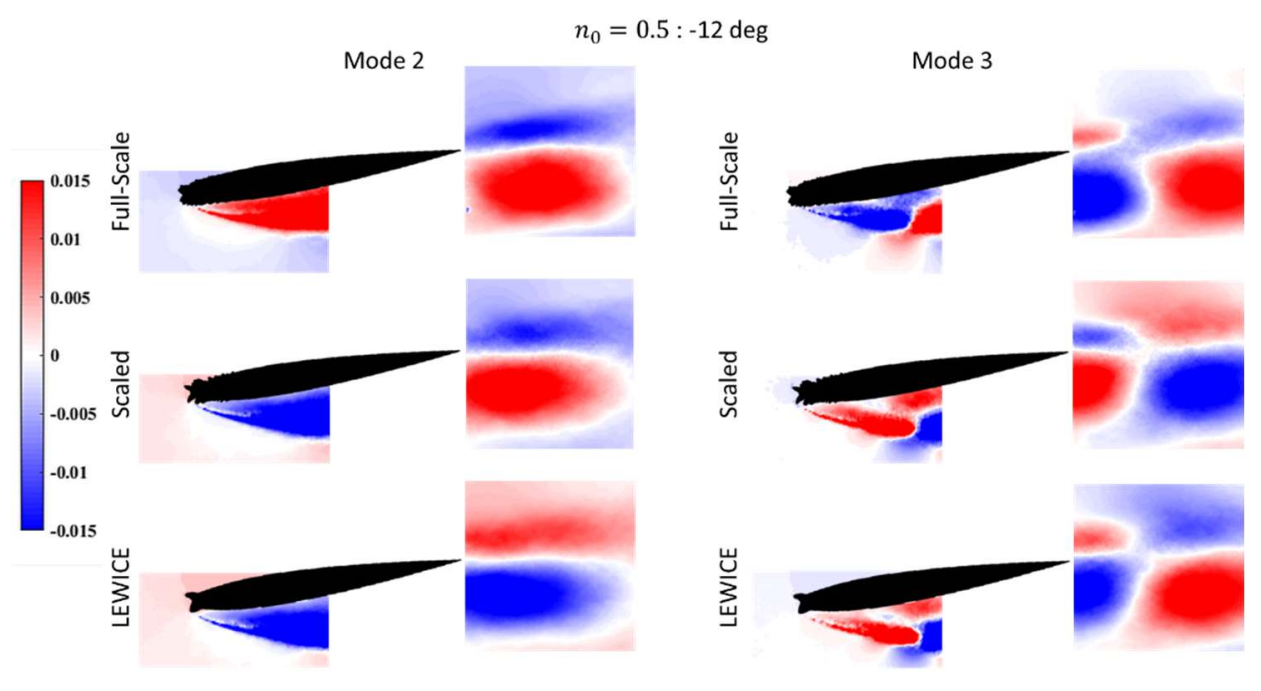

Figure 53: Second and third POD U-velocity correlation modes for $\boldsymbol{n}_{0}=0.5$ ice accretion cases

It can be seen that the LEWICE and scaled ice shapes differ from the full-scale ice shape at positive angles of attack, but are fairly characteristic of the full-scale at negative angles of attack. At $12^{\circ}$, none of the ice shapes show on-body flow structures in the second mode, but the LEWICE and scaled ice shapes show vortex shedding structures in the wake. For the rest of the angles of attack presented, no coherent structures are found in the field of view in the second mode. The wake size and the amount of separation shown in the second mode corresponds with the results of the mean U-velocity flow field analysis, with the full-scale consistently having a smaller wake and less separation than the LEWICE and scale ice shapes.

On-body coherent structures relating to vortex shedding structures can be seen throughout the third mode, except at $0^{\circ}$. The coherent structures at positive $12^{\circ}$ of the LEWICE and scaled ice shapes have slightly larger length scale, whereas at negative $12^{\circ}$ the coherent structures are highly similar for all three ice shapes.

The slightly shorter upper horn of the full-scale ice shape results in shorter third mode structures than the LEWICE and scaled ice shapes at $6^{\circ}$. At $-6^{0}$, the third mode structures of the full-scale ice shape are slightly smaller than the LEWICE and scaled ice shapes. This is due to the 
lower horn geometries of the three ice shapes being highly similar with minimal difference in height.

The $0^{\circ}$ case is the only angle of attack at which the LEWICE and scaled ice shapes do not show high similarity. The scaled and full-scale, however, are similar at $0^{\circ}$. Both the scaled and fullscale have small on-body structures. Whereas, the LEWICE ice shape has larger on-body structures with possible shedding being present in the wake. As mentioned in previous sections, this is due to the drastic geometry change of the LEWICE ice shape due to the absence of any feather region ice accretion.

\subsubsection{V-velocity Correlations}

Figure 54 shows the second and third modes with respect to the V-velocity of ice shapes collected at $n_{0}=0.5$. As shown, shedding is present and coherent structures can be seen throughout the wake profiles for all ice shapes. The structures are easily compared based on size. As was the case of the U-velocity modes, the V-velocity modes show strong similarity between the LEWICE and scaled ice accretions, the trend which is seen in the aerodynamic coefficients, vorticity, and Reynolds stress contours.

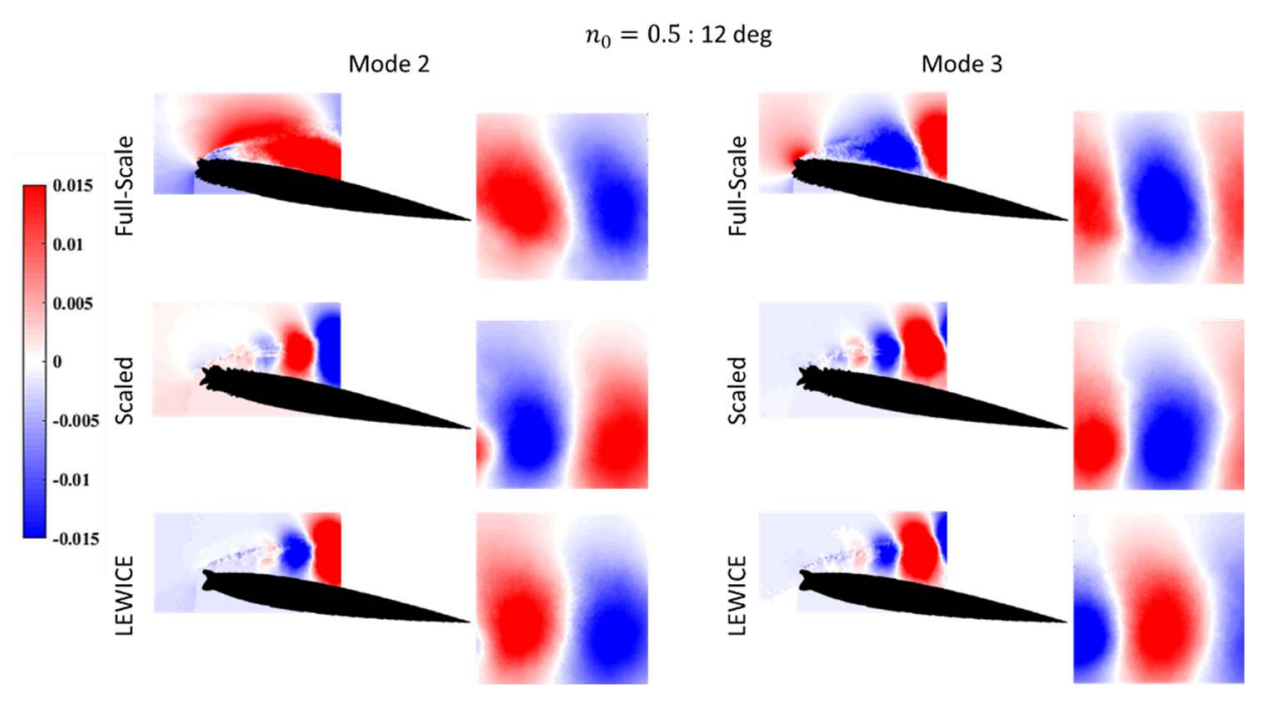



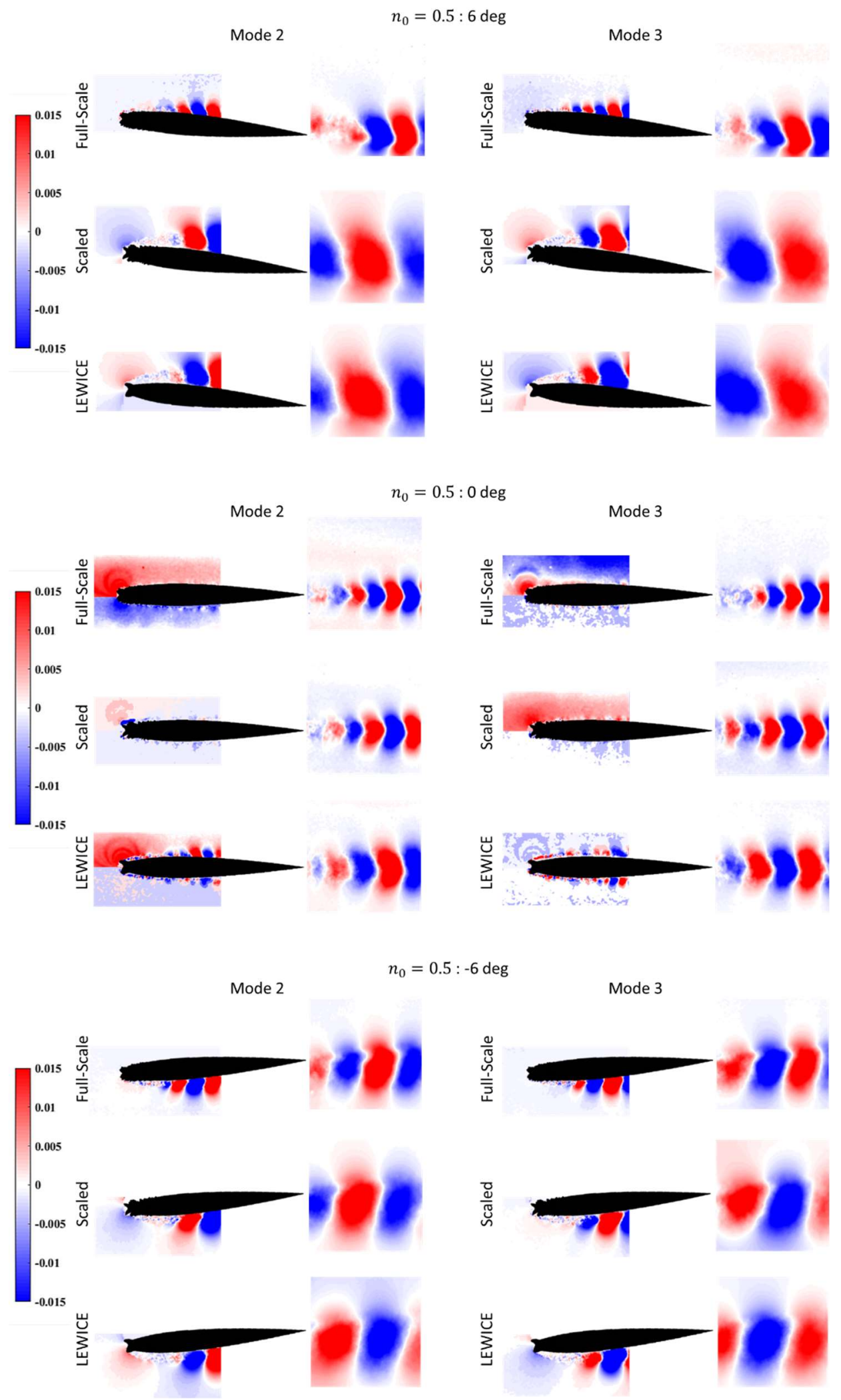


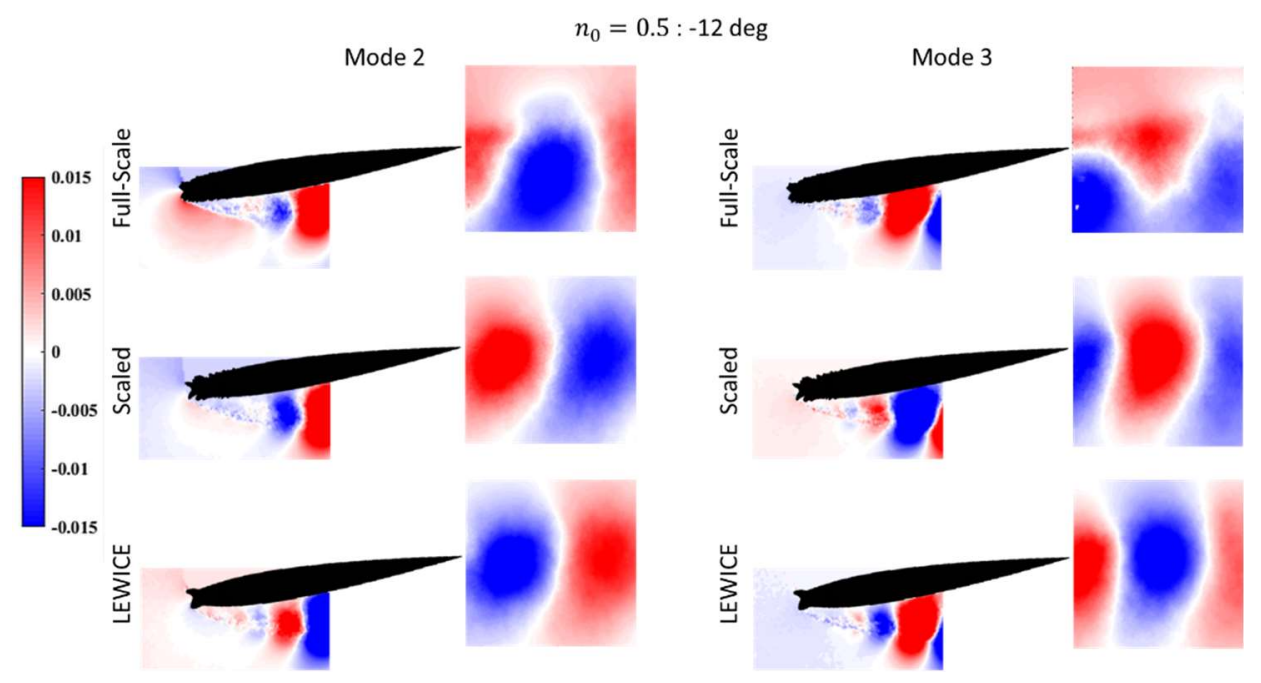

Figure 54: Second and third POD V-velocity correlation modes for $n_{0}=0.5$ ice accretion cases

The structures in the near wake at $12^{\circ}$ angle of attack are similar for both modes for all three ice shapes. However, the significant differences exist in the boundary layer POD modes. The coherent structures shown in the second and third modes have larger length scales than the other two cases. The LEWICE and scaled ice shapes both generate structures which are similar in size. This trend continues at $6^{0}$, where the full-scale ice shape produces smaller coherent structures at the same energy level both on the surface and in the wake when compared to the other two cases.

As expected, based on the greater Reynolds stress magnitude, the LEWICE ice shape has the largest coherent structures of the three ice shapes at $0^{0}$ and $-6^{0}$ angles of attack. For a given energy level, the full-scale ice shape produces coherent structures with shorter length scales than the LEWICE and scaled ice shapes at these angles of attack. At $-12^{\circ}$ the size of the on-body structures for all three ice shapes are comparable for both the second and third modes.

\subsubsection{Modal Flow Field Energy Content}

The energy present in each of the first four U-velocity and V-velocity modes are plotted in Figures 55 and 56 for the $n_{0}=0.5$ ice accretions, respectively, with the first mode being the mean flow. 


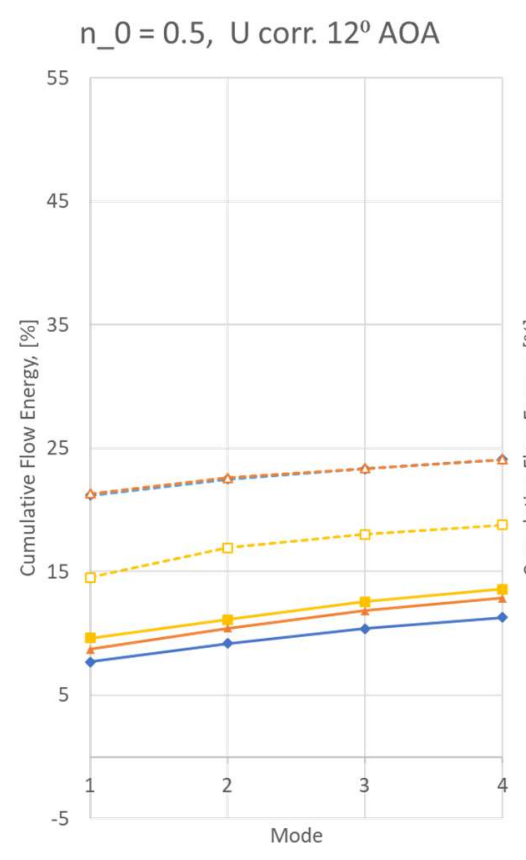

n_0 $=0.5$, U corr. $6^{\circ} \mathrm{AOA}$

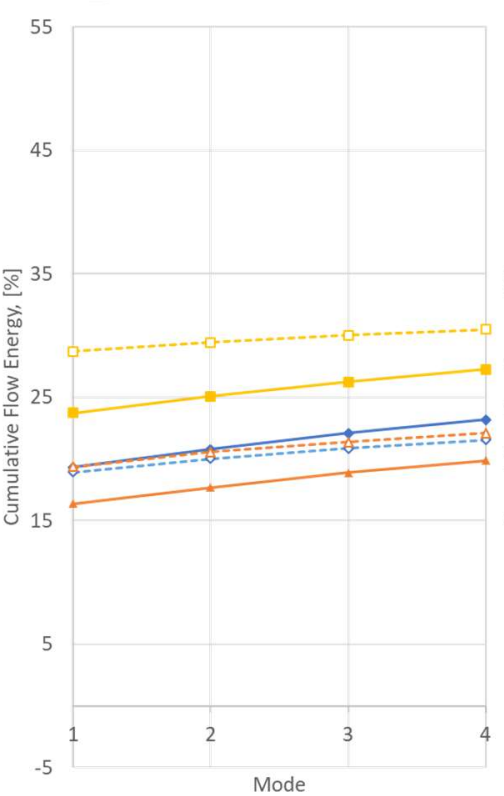

$\mathrm{n} \_0=0.5, \mathrm{U}$ corr. $0^{\circ} \mathrm{AOA}$

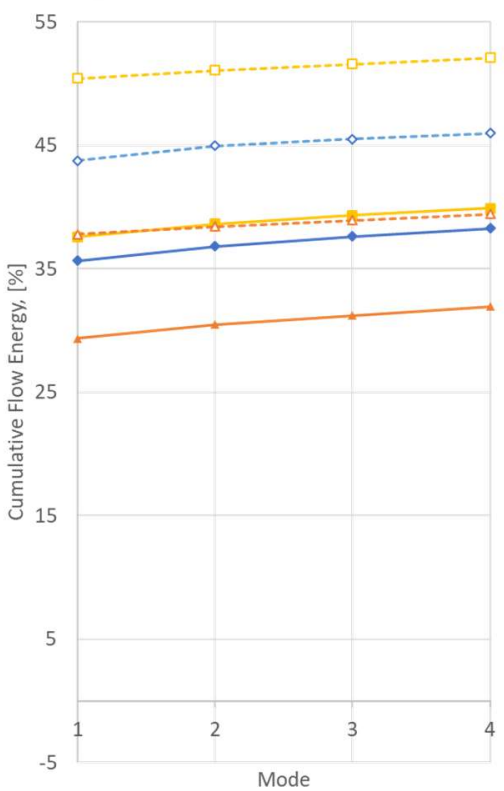

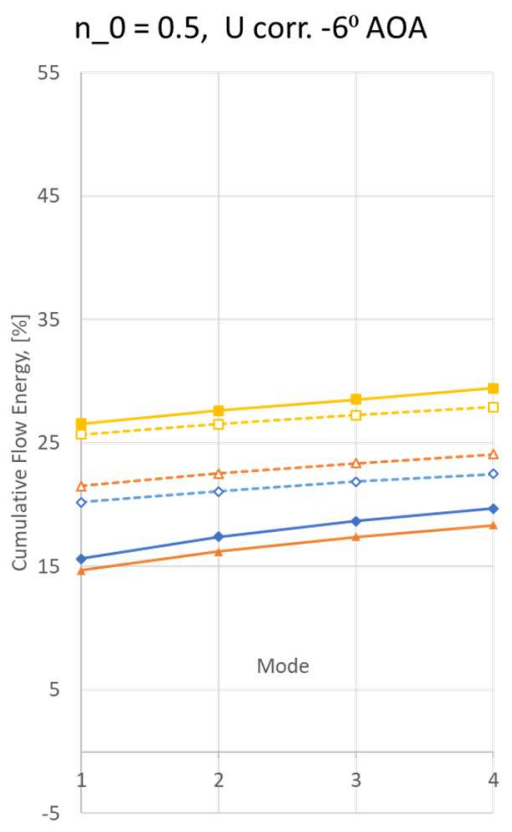

n_0 $=0.5, U$ corr. $-12^{\circ}$ AOA

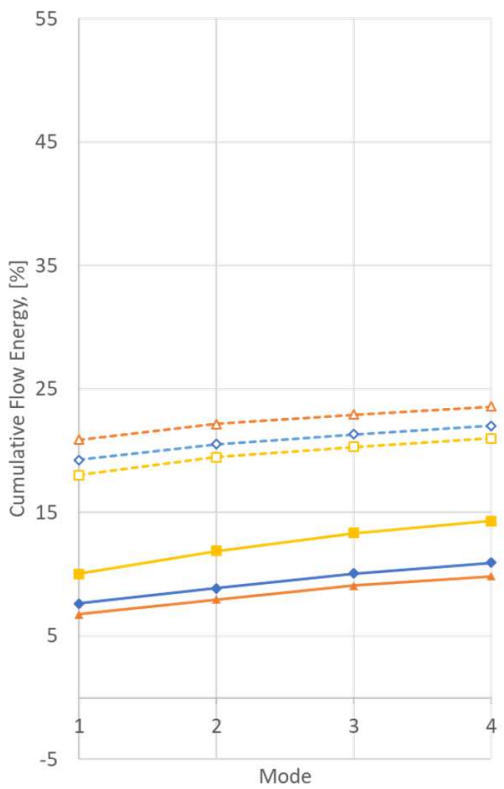

- Full-scale, Free Shear V corr. —- Scaled, Free Shear V corr.

$\longrightarrow$ LEWICE, Free Shear V corr.

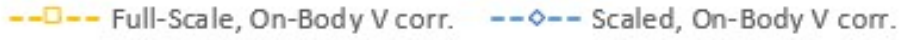
-- $\triangle--$ LEWICE, On-Body V corr.

Figure 55: Cumulative flow field energy content for first four U-velocity correlation modes for $n_{0}=0.5$ ice accretion cases 


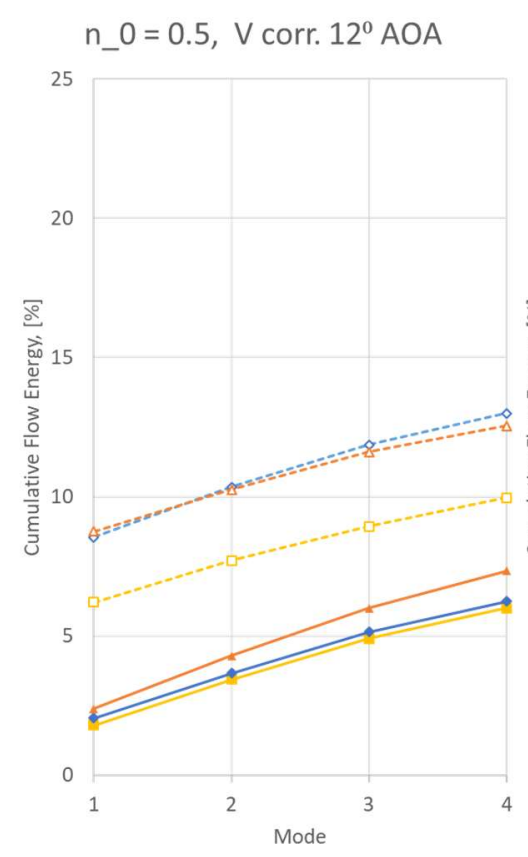

$\mathrm{n} \_0=0.5, \mathrm{~V}$ corr. $6^{0} \mathrm{AOA}$

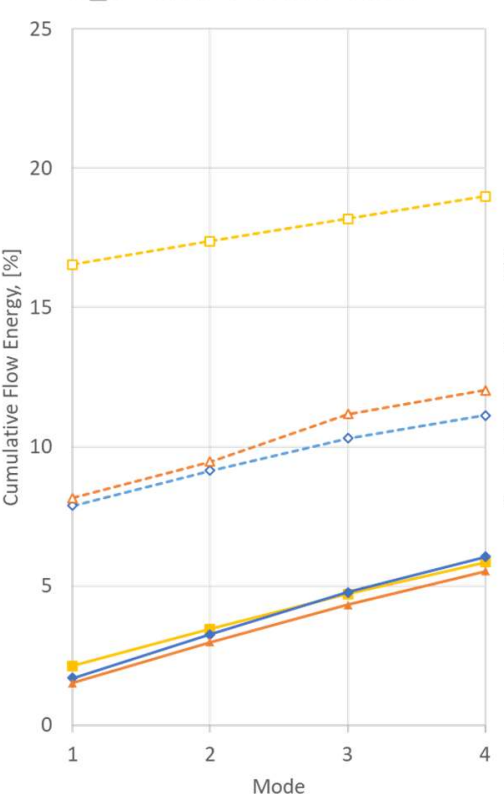

n_o $=0.5, v$ corr. $0^{\circ} \mathrm{AOA}$

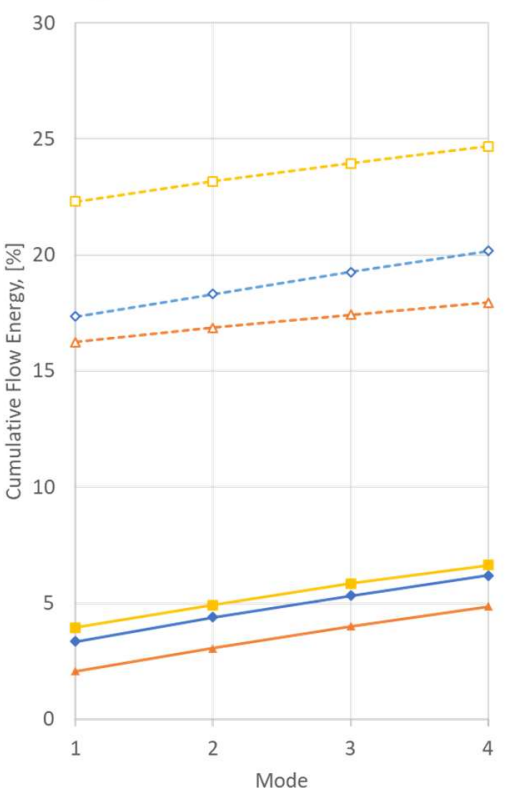

n_0 $=0.5$, V corr. $-6^{0} \mathrm{AOA}$

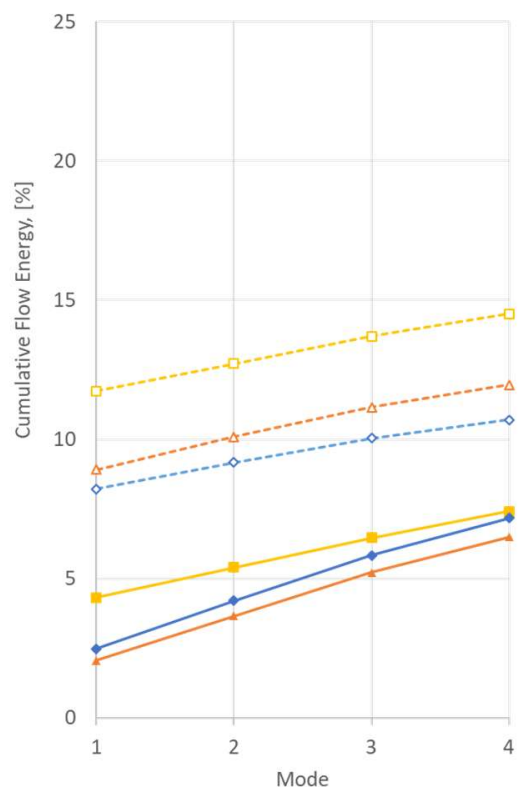

$\mathrm{n} \_0=0.5$, V corr. $-12^{\circ} \mathrm{AOA}$

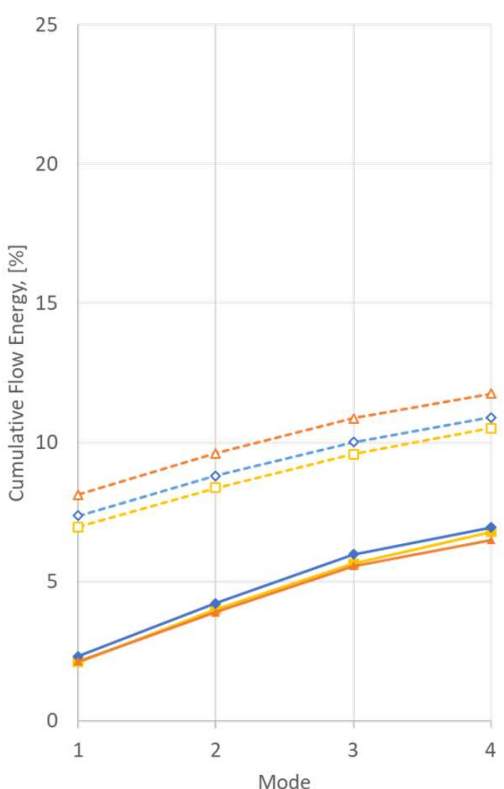

- Full-scale, Free Shear V corr. — Scaled, Free Shear V corr.

$\longrightarrow$ LEWICE, Free Shear V corr.

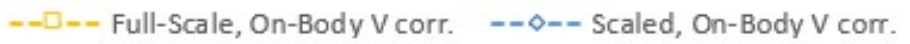

$--\triangle--$ LEWICE, On-Body V corr.

Figure 56: Cumulative flow field energy content for first four $\mathrm{V}$-velocity correlation modes for $n_{0}=0.5$ ice accretion cases 
Similar to the first set, the deviations in energy content for the second through fourth modes is mostly $<1 \%$ between the three different ice shapes. Unlike the first set of ice shapes, there are significant deviations seen in the energy content of both the $\mathrm{U}$ and $\mathrm{V}$-velocity mean flows between the three ice shapes. The largest deviation again occurs at $0^{\circ}$. The LEWICE and scaled ice shape modal energy contents are highly similar, never varying greater than $2 \%$, besides at $0^{\circ}$. At $0^{\circ}$, the scaled ice shape flow has greater than $6 \%$ more flow energy present in the mean U-velocity flow. This was also the angle of attack at which the coherent structures varied the most between the LEWICE and scaled ice shapes.

In comparison to the full-scale ice shape, the scaled ice shape energy content in the first mode (the mean flow) varies significantly at multiple angles of attack. The flow energy present in the mean U-velocity flow varies by greater than $5 \%$ at $12^{\circ}$ and $-6^{\circ}$ and greater than $8 \%$ at both $6^{\circ}$ and $0^{\circ}$. The mean $\mathrm{V}$-velocity also varies by greater than $8 \%$ at $6^{\circ} \mathrm{AOA}$ and $\sim 5 \%$ at $0^{\circ}$. With the minimal difference in energy content between the LEWICE and scale ice shapes, besides at $0^{\circ}$, the LEWICE ice shape deviates at similar magnitudes from the full-scale ice accretion. At $0^{\circ}$, the deviation from the full-scale in the energy present in the mean $\mathrm{U}$ and $\mathrm{V}$-velocity flows is greater than $12 \%$ and greater than $6 \%$, respectively.

The LEWICE and scaled ice shapes varied from the full-scale ice shape except at high angles of attack. The coherent structures produced by each of the LEWICE and scaled ice shapes were consistently larger than those of the full-scale ice. The variation was also expressed by the energy modes. Therefore, the LEWICE and scaled ice shapes were both uncharacteristic of the fullscale ice shapes corresponding with the trend seen in the aerodynamic coefficients, vorticity, and Reynolds stress contours, again showing the LEWICE ice shape is uncharacteristic of the full-scale ice shape.

\subsection{Summary of Chapter VI}

The results of the POD flow field analysis corroborated the results of the aerodynamic coefficients, vorticity, and Reynolds stress contours. For the $n_{0}=0.3$ ice accretions, the scaled ice 
accretion produced both U-velocity and V-velocity correlation coherent structure of similar length scale to the full-scale with the exception to the $6^{0}$ angle of attack case.

The deviation at the midrange positive angles of attack is due to the large, aft positioned upper feather region ice accretion of the scale ice shape and has been shown throughout the research presented. The LEWICE ice shape only produced coherent structure of similar size at $-12^{\circ}$, therefore once again, being uncharacteristic of the full-scale ice shape for the $n_{0}=0.3$ ice accretions.

With respect to the $n_{0}=0.5$ ice accretions, the scaled and LEWICE ice shapes were less characteristic of the full-scale than shown in the PIV flow field analysis of Chapter V. The energy content in the mean velocity and the length scales of the coherent structures deviated across most of the angle of attack range. This reiterates the inconsistency of the recommended ice shape scaling method to reproduce an ice shape characteristic of the full-scale and again suggests a possible temperature/stagnation freezing fraction constraint for use in SLD conditions. The LEWICE predicted ice shapes were again uncharacteristic of the full-scale ice shape for both the $n_{0}=0.3$ and $n_{0}=0.5$ ice accretions. 


\section{CHAPTER VII}

\section{CONCLUSIONS}

\subsection{Conclusions}

The results from the current investigation provided a low Reynolds number aerodynamic verification across a wide range of angles of attack of the ice accretions generated by Anderson and Tsao's scaling method [1] and the LEWICE predictive software for two different stagnation freezing fraction, $n_{0}$ under Supercooled Large Droplet (SLD) conditions. Experimental investigations were performed in the University of Dayton Low Speed Wind Tunnel to assess different aspects of the aerodynamics associated with the scaling method and LEWICE produced ice accretions compared to an ice accretion collected on a full-scale model, a model with a 1:1 representation of a desired body of interest. Force-based experimentation was utilized to evaluate the lift and drag coefficients as a function of angle of attack, allowing for the assessment of the preservation of the aerodynamic penalties associated with each of the ice shapes; including reduced stall angle, increased drag, and disruption of the flow field. Particle Image Velocimetry (PIV) was leveraged to evaluate the mean and fluctuating components of velocity in the leading edge and near wake regions. The near wake momentum deficit obtained from PIV was used to estimate the drag coefficient for the different ice shapes. The PIV interrogation regions allowed study of the mean streamwise velocity, the vorticity, and the Reynolds stress over and near the ice accretion as well as at the near wake. These flow properties were used to determine if the aerodynamics is preserved across all three ice shapes. Along with the Reynolds stress, the fluctuating component of the velocity was evaluated for coherent structures using a modal analysis known as proper orthogonal decomposition.

\subsubsection{Ice Shape Scaling Method Aerodynamic Verification Conclusion}

The results showed inconsistency in the ability of the Anderson and Tsao [1] recommended ice shape scaling method of producing an ice accretion which maintains the aerodynamics and the 
associated flow physics with that of a full-scale ice shape. For the ice accretions collected with a stagnation freezing fraction of $n_{0}=0.3$, the scaled ice shape showed very similar behavior as the full-scale ice shape, except for small deviations at the midrange positive angles of attack, as evidenced by the force-based and PIV results. It was clear that the large feather region on the upper surface of the scaled ice shape was the main cause of the deviation from the full-scale at the midrange positive angles of attack. The mean streamwise velocity, vorticity, and Reynolds stress contours around the leading-edge showed a clear separation point at the tip of the upper surface feather region ice accretion causing the deviation from the full-scale ice shape. Overall the scaled ice shape collected with a $n_{0}=0.3$ was mostly characteristic of the corresponding full-scale ice shape.

This agreement between the full-scale and the scaled ice shape was not observed in the $n_{0}=0.5$ cases. The similar lower horn geometries of the scaled and full-scale ice shapes produced similar flow physics at negative angles of attack. However, this was not the case at positive angles of attack, where the geometric variation of the upper horns caused the aerodynamics between the scaled and full-scale ice shapes to vary. The scaled ice shape also showed earlier stall at both positive and negative angles of attack, therefore, not preserving the aerodynamic penalty of early stall. Overall, the scaled ice shape collected with a $n_{0}=0.5$ was uncharacteristic of the corresponding full-scale collected ice shape.

The difference in the evaluation of the scaled and full-scale ice shapes for the SLD conditions evaluated in this research show inconsistency in the ability of the Anderson and Tsao [1] recommended ice shape scaling method to reproduce an ice shape aerodynamically characteristic of the full-scale ice accretion in SLD icing conditions. Therefore, it can be concluded that the ice accretion generated by the scaling method does not deliver the same aerodynamic effect as the full-scale ice shape. Rather, the results suggest a possible stagnation freezing fraction constraint for the scaling method's use in SLD conditions, as this was the primary icing collection parameter difference between the ice shapes collected with a $n_{0}=0.3$ and $n_{0}=0.5$. The results 
also expressed the necessity for better preservation of the feather region ice accretion geometry and frontal horn height, as these provided the largest deviations in the aerodynamics of the different ice shapes. This along with the inconsistency of the scaling method to produce an ice shape that preserved the associated aerodynamics with the full-scale ice accretion between the two stagnation freezing fraction cases suggest the necessity of further refinement of the recommended ice shape scaling method for SLD conditions.

\subsubsection{LEWICE Software Aerodynamic Verification Conclusion}

Unlike in the case of the scaling method, the LEWICE predicted ice shapes were consistently uncharacteristic of the full-scale ice accretion. For the LEWICE ice shape predicted based on the icing parameters of the full-scale ice accretion collected with a $n_{0}=0.3$, the LEWICE ice shape was aerodynamically uncharacteristic of the full-scale ice shape throughout the angle of attack range, with a small exception at midrange positive angles of attack. The LEWICE ice shape for the most part failed to reproduce the aerodynamic effect of the full-scale ice shape, showing later stall and for most of the angle of attack range significantly lower drag. This carried into the flow field analysis and was expressed by consistently producing less transfer of momentum due to turbulence in the flow and producing smaller coherent structures. Overall, the LEWICE ice shape simulated with a $n_{0}=0.3$ was uncharacteristic of the corresponding full-scale collected ice shape.

The LEWICE predicted ice shape with a $n_{0}=0.5$ was also uncharacteristic of the corresponding full-scale ice shape. Though showing some aerodynamic similarity to the full-scale at negative angles of attack, the modal analysis and early stall showed deviation at this range between the two ice shapes. For the rest of the angle of attack range, the LEWICE ice shape consistently produced different flow characteristics than the full-scale ice accretion. This was expressed across all experimentation.

Overall, the LEWICE software was consistently unable to predict an ice shape aerodynamically characteristic of the full-scale ice accretion for the SLD conditions evaluated in this thesis. Therefore, the LEWICE software fails to be aerodynamically verified for SLD 
conditions. This is expressed by the inconsistency in the under or over prediction of the full-scale icing effect on the aerodynamics. LEWICE and similar software are typically used during the aircraft design process. However, the results presented suggest that this is poor practice since the LEWICE does not consistently predict an ice shape with a similar or greater effect on the associated aerodynamics, as shown by the $n_{0}=0.3$ case. This could result in a non-conservative approach during the aircraft design process and could lead to the necessity of redesign following experimental evaluation. The LEWICE software is still useful for studying icing with respect to the main ice formation, but experimentation is still required for studying SLD conditions.

\subsection{Future Work}

The verification performed in this thesis focused on two-dimensional ice geometries collected through tracing the ice shape at the model midspan for the scale and full-scale models in the NASA IRT and predicted through the two-dimensional prediction condition of the LEWICE software. As NASA Glenn IRT begins to use technology to record ice shapes three-dimensionally, further work is needed to assess the effect of the spanwise variation of the ice shape on the aerodynamic verification of the scaling method and LEWICE three-dimensional predictions. The spanwise variation may cause better agreement between the scaled and full-scale ice shapes, if the geometric differences are the most extreme at midspan location where the two-dimensional tracings were collected, however this is unknown. The research presented provides template for experimentally evaluating the aerodynamics of different ice accretions, allowing for further SLD conditions and of three-dimensionally recorded and predicted ice accretions to be studied. This in turn will allow for verification of ice shape scaling methods and ice shape predictive software to be assessed for aerodynamic verification. It is important to note that multiple spanwise positions will need to be evaluated for three-dimensional ice shapes to fully understand the effect of the spanwise variation in the ice shapes and the to fully assess the aerodynamic verification based on three-dimensional ice accretions. 


\section{REFERENCES}

[1] Anderson, David N. and Tsao, Jen-Ching., "Ice Shape Scaling for Aircraft in SLD Conditions," NASA/CR-2008-215302, September 2008.

[2] Wright, William, “User's Manual for LEWICE Version 3.2,” NASA/CR-2008-214255, November 2008.

[3] Code of Federal Regulations, Title 14, Chapter I, Federal Aviation Administration, Department of Transportation, Part 25, "Airworthiness Standards: Transport Category Aircraft," Appendix C and Appendix O, U.S. Government Printing Office, Washington D.C., revised as of October 2015.

[4] Ide, Robert and Oldenburg, John, "Icing Cloud Calibration of the NASA Glenn Icing Research Tunnel," NASA/TM-2001-210689, 2001.

[5] Anderson, David N., "Manual of Scaling Methods," NASA/CR-2004-212875, March 2004.

[6] Ruff, Gary A., “Analysis and Verification of the Icing Scaling Equations,” AEDC-TR-85-30, Vol. 1 (rev), March 1986.

[7] NASA. Icing Research Tunnel: Facility Overview. 21 January 2020. July 2020.

[8] Bragg, M. B., Broeren, A. P., and Blumenthal, L. A., "Iced-Airfoil Aerodynamics," SAE Paper 2003-01-2098, June 2003.

[9] Anderson, D. N., Shin J., "Characterization of Ice Roughness from Simulated Icing Encounters,” AIAA Paper 97-0052, 1997.

[10] Blumenthal, LA.. Surface Pressure Measurement on a Three-Dimensional Ice Shape. MS Thesis, Department of Aerospace Engineering. University of Illinois, Urbana, IL, 2005.

[11] Lee, S., Bragg, M. B., "Effects of Simulated-Spanwise Ice Shapes on Airfoils: Experimental Investigation,” AIAA Paper 99-0092, 1999.

[12] Gurbacki, Molly M. and Bragg, Michael B., "Unsteady Flowfield about an Iced Airfoil," AIAA Paper 2004-562, January 2004. 
[13] Blumenthal, Leia A., Busch, Greg T., Broeren, Andy P. and Bragg, Michael B., "Issues in Ice Accretion Aerodynamic Simulation on a Subscale Model,” AIAA Paper 2006-262, January 2006.

[14] Kim, Han S. and Bragg, Michael B., "Effects of Leading-Edge Ice Accretion Geometry on Airfoil Performance," AIAA Paper 99-3150.

[15] Lee, Sam and Bragg, Michael B., "Investigation of Factors Affecting Iced-Airfoil Aerodynamics," Journal of Aircraft, Vol. 40, No. 3, May 2003.

[16] Roberts, A. B., "Calculation of Laminar Separation Bubbles and Their Effect on Airfoil Performance," AIAA Journal 1980, 18(1): 25-31, 1980.

[17] Bragg, M. B., Khodadoust, A, Spring, S. A., "Measurements in a Leading-Edge Separation Bubble due to a Simulated Airfoil Ice Accretion,” AIAA Journal 1992, 30(6): 1462-1467.

[18] Olsen, William and Newton, James, "Experimental and Analytical Evaluation of Existing Icing Scaling Laws,” unpublished draft of NASA Technical Memorandum, 1986.

[19] Langmuir, Irving and Blodgett, Katherine B., “A Mathematical Investigation of Water Droplet Trajectories,” Army Air Forces Technical Report No. 5418, February 1946.

[20] Anderson, David N., “Acceptable Tolerances for Matching Icing Similarity Parameters in Scaling Applications,” AIAA Paper 2001-0832, January 2001.

[21] Wright, William B., "Comparison of LEWICE and GlennICE in the SLD Regime," NASA/TM-2008-215174, September 2008.

[22] Wright, William B. and Porter, Christopher E., "Validation Process for LEWICE by Use of a Navier-Stokes Solver,” https://ntrs.nasa.gov/search.jsp?R=20170007203, June 2017.

[23] Gunasekaran, Sidaard. Relationship Between the Free Shear Layer, the Wingtip Vortex and Aerodynamic Efficiency. Doctoral Dissertation. Dayton: University of Dayton Libraries, 2016.

[24] Anderson, J. D., Fundamentals of Aerodynamics. Second Edition, McGraw-Hill, Boston, 1991. 
[25] Lumley, J. L., "The Structures of Inhomogeneous Turbulent Flows," Proceedings of the International Colloquium on the Fine Scale Structure of the Atmosphere and Its Influence on Radio Wave Propagation, edited by A. M. Yaglam and V.I. Tatarsky, Doklady Akademii Nauk SSSR, Nauka Moscow, 1967.

[26] Taira, Kunihiko, Brunton, Steven L., Dawson, Scott T. M., Rowley, Clarence W., Colonius, Tim, Mckeon, Beverly J., Schmidt, Oliver T., Gordeyev, Stanislav, Theofilis, Vassilios and Ukeiley, Lawrence S., "Modal Analysis of Fluid Flows: An Overview,” AIAA Journal, Vol. 55, No. 12, December 2017.

[27] Sirovich, L., "Turbulence and the Dynamics of Coherent Structures, Parts I-III," Quarterly of Applied Mathematics, Vol. 45, No. 3, 1987, pp. 561-571.

[28] Ananda, G. K., Sukumar, P.P., and Selig, M.S., "Measured Aerodynamic Characteristics of Wings at Low Reynolds Numbers,” Department of Aerospace Engineering. University of Illinois, Urbana, IL, 2015.

[29] ATI Industrial Automation. F/T Sensor: Gamma.2020. January 2020.

[30] Particle Image Velocimetry - Dantec Dynamics

[31] Raffel, M., Willert, C. and Kompenhans, J., Particle Image Velocimetry: A Practical Guide, Springer, Berlin, Heidelberg, New York, 1998.

[32] Griffin Motion. Rotary Stages. 2020. January 2020.

[33] TSI. DP-CALC Micromanometer 5825. 2020. January 2020.

[34] Lazar, Eli, DeBlauw, Bradley, Glumac, Nick, Dutton, Craig and Elliot, Gregory, “A Practical Approach to PIV Uncertainty Analysis,” AIAA Paper 2010-4355, July 2010.

[35] Chatterjee, A., "An Introduction to the Proper Orthogonal Decomposition," Current Science, 78(7):808-817, 2000.

[36] Chen, H., Reuss, D. L., Hun, D. L. S., and Sick, V., “A Practical Guide for Using Proper Orthogonal Decomposition in Engine Research," International Journal of Engine Research, 14(4):307-319, 2012. 
[37] Olsen, William, Shaw, Robert and Newton, James., "Ice Shapes and the Resulting Drag Increase for a NACA 0012 Airfoil," NASA/TM-83556, January 1984. 


\section{APPENDIX A}

LEWICE Ice Shape Input Files Based on Full-Scale Icing Parameters

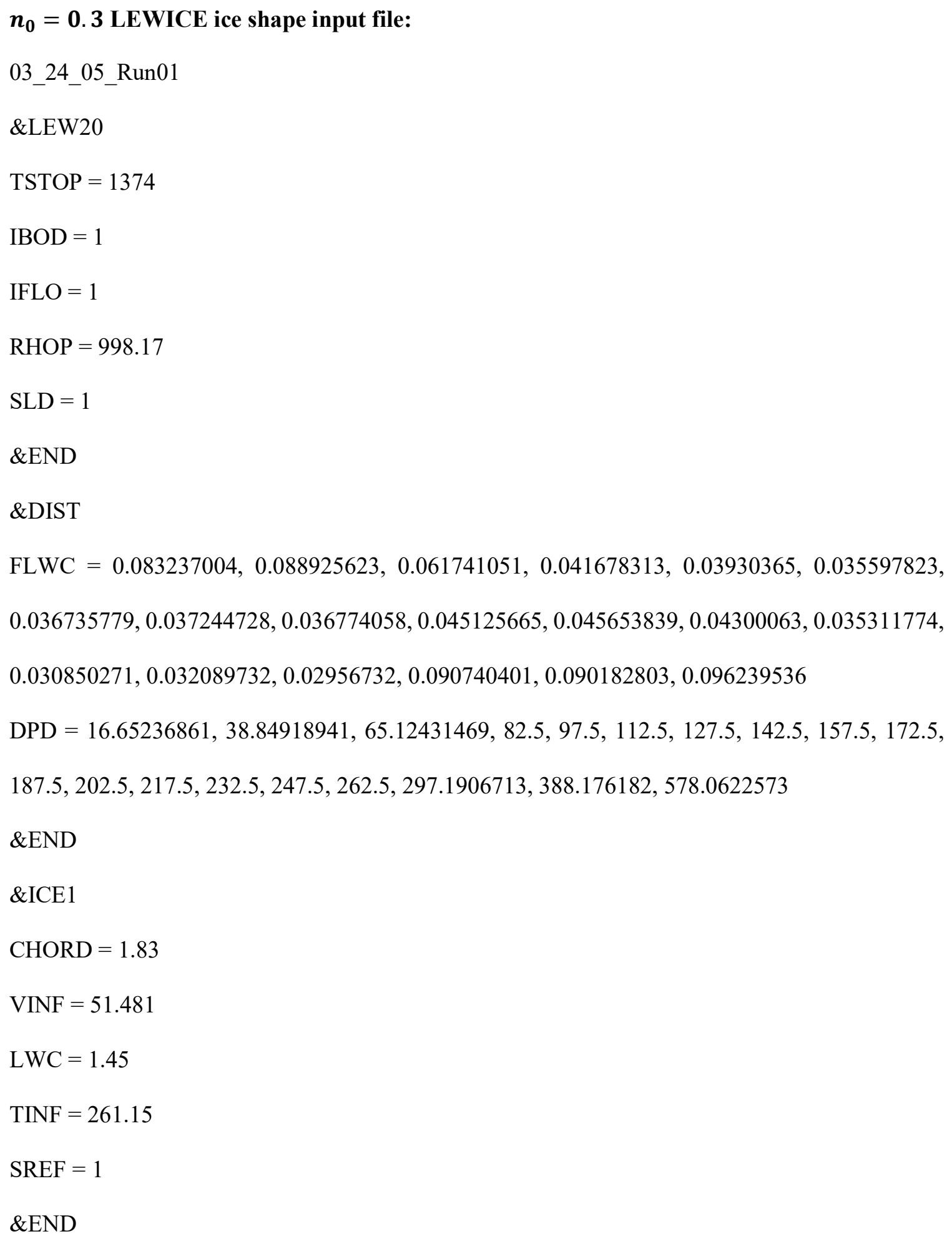




\author{
\&LPRNT \\ \&END \\ \&RDATA \\ \&END \\ $\&$ BOOT \\ \&END
}

$n_{0}=0.5$ LEWICE ice shape input file:

03_24_05_Run03

\&LEW20

$\mathrm{TSTOP}=1374$

$\mathrm{IBOD}=1$

IFLO $=1$

RHOP $=993.55$

$\mathrm{SLD}=1$

\&END

\&DIST

$\mathrm{FLWC}=0.083237004,0.088925623,0.061741051,0.041678313,0.03930365,0.035597823$, $0.036735779,0.037244728,0.036774058,0.045125665,0.045653839,0.04300063,0.035311774$, $0.030850271,0.032089732,0.02956732,0.090740401,0.090182803,0.096239536$ $\mathrm{DPD}=16.65236861,38.84918941,65.12431469,82.5,97.5,112.5,127.5,142.5,157.5,172.5$ $187.5,202.5,217.5,232.5,247.5,262.5,297.1906713,388.176182,578.0622573$

\&END

$\&$ ICE1

$\mathrm{CHORD}=1.83$

$\mathrm{VINF}=51.481$ 


LWC $=1.45$
TINF $=252.15$
SREF $=1$
\&END
\&LPRNT
\&END
\&RDATA
\&END
\&BOOT
\&END




\section{APPENDIX B}

MVD $170 \mu \mathrm{m}$ NASA Glenn Icing Research Tunnel Droplet Distribution Data

Cumulative volume droplet distribution used on LEWICE input files.

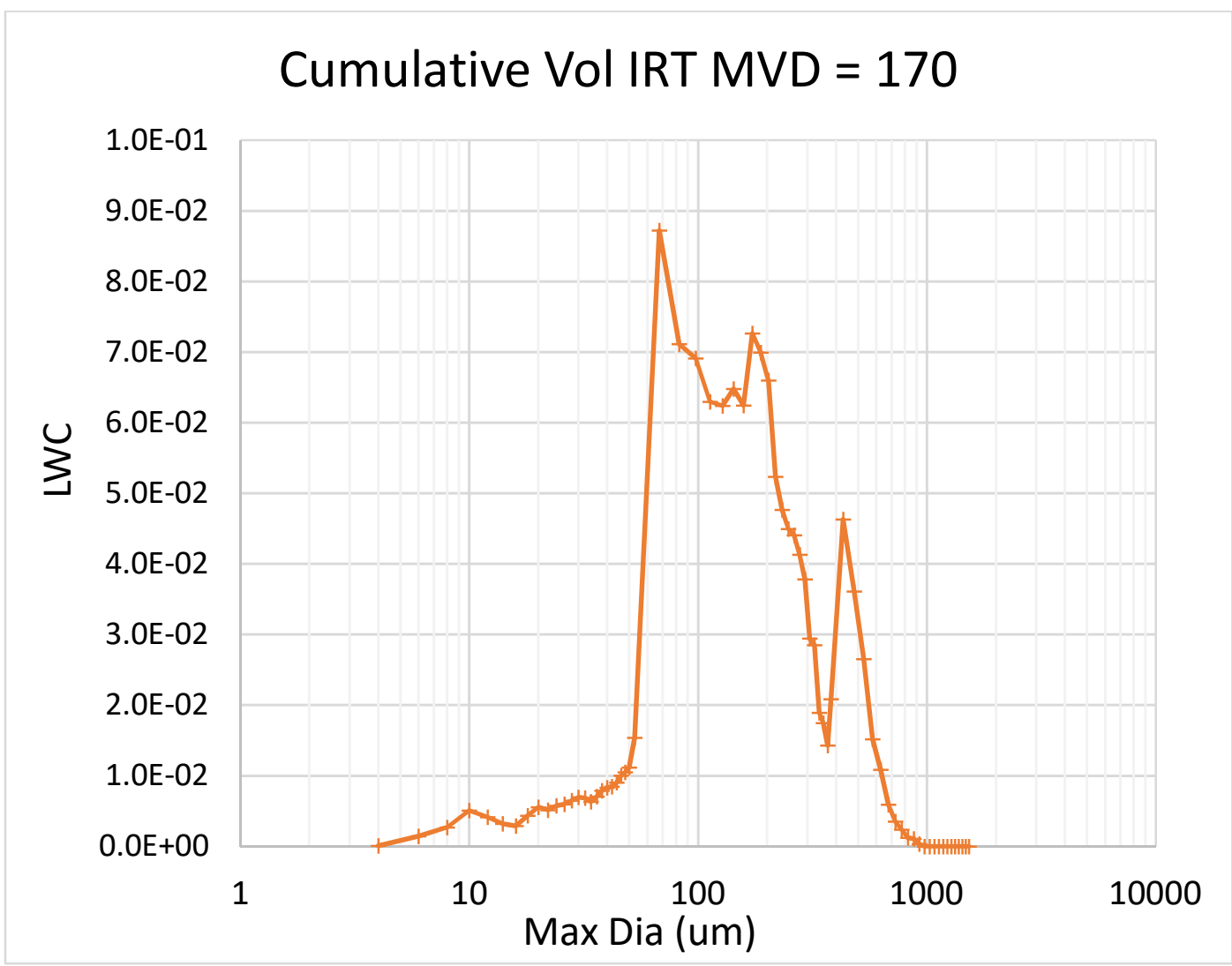

